Supporting Information

\title{
Visible-Light-Induced, Metal-Free Carbene Insertion into B-H Bonds between Acylsilanes and Pinacolborane
}

\author{
Jian-Heng Ye, Linda Quach, Tiffany Paulisch and Frank Glorius \\ Organisch-Chemisches Institut, Westfälische Wilhelms-Universität Münster, \\ Corrensstraße 40, 48149 Münster, Germany
}

glorius@uni-muenster.de 


\section{Contents}

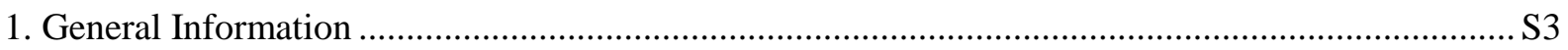

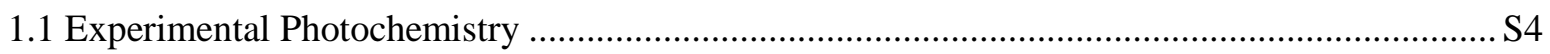

2. Experimental Procedures and Characterization Data …….............................................................. S5

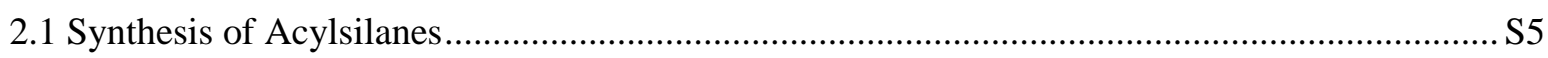

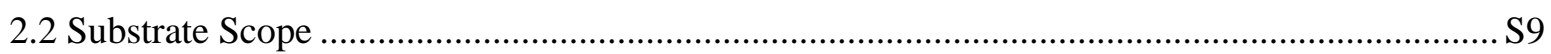

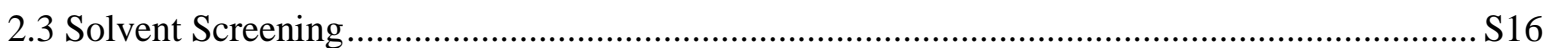

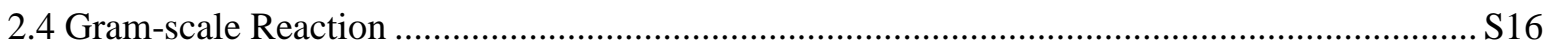

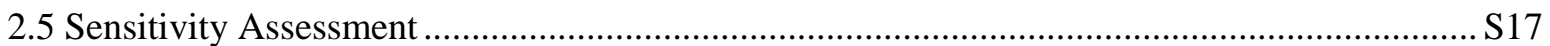

4. Characterization Studies with UV/Vis Spectroscopy …................................................................... 18

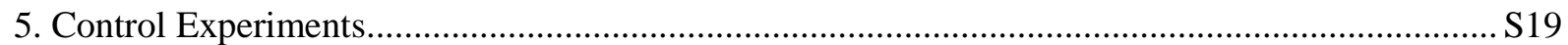

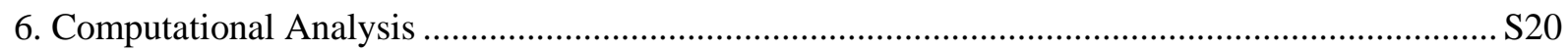

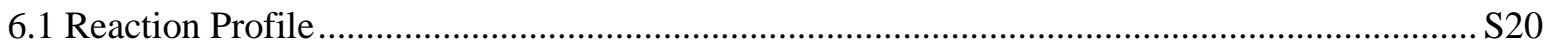

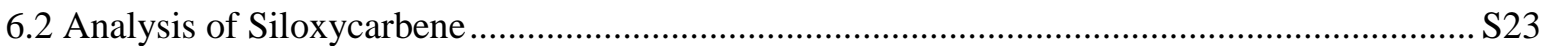

6.3 Further Investigation of Pathway towards Intermediate X........................................................ S29

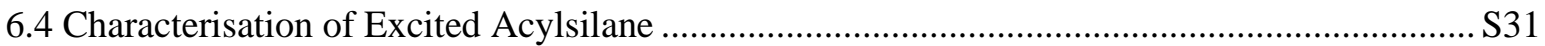

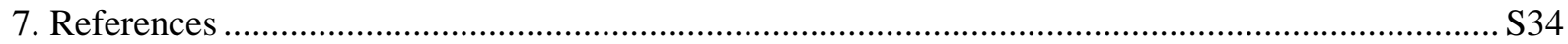

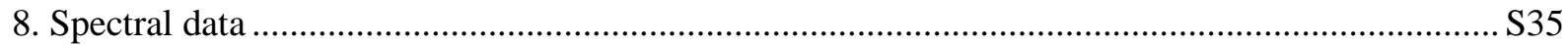




\section{General Information}

All reactions were carried out under an atmosphere of argon in oven-dried glassware using standard Schlenk technique, unless otherwise noted. Solvents were purified by distillation over standard drying agents, stored over molecular sieves and transferred under argon. Except where stated, all starting materials were commercially available and used without further purification. Acylsilanes were checked for purity by TLC before use and purified by distillation if impurities were observed.

NMR-spectra were recorded on a Bruker ARX-300, AV-300, AV-400 MHz or on a Varian Associated, Varian 600 unity plus spectrometer. All spectral data was acquired at $295 \mathrm{~K}$. Chemicals shifts $(\delta)$ are quoted in parts per million (ppm). The following residual solvent signals were used as references for ${ }^{1} \mathrm{H}$ and ${ }^{13} \mathrm{C} \mathrm{NMR}$ spectra: $\mathrm{CDCl}_{3}, \delta_{\mathrm{H}} 7.26 \mathrm{ppm}$, $\left.\delta_{\mathrm{C}} 77.16 \mathrm{ppm} ; \mathrm{CD}_{2} \mathrm{Cl}_{2}, \delta_{\mathrm{H}} 5.32 \mathrm{ppm}, \delta_{\mathrm{C}} 54.00 \mathrm{ppm}\right)$. Coupling constants $(J)$ are reported in Hertz $(\mathrm{Hz})$ to the nearest $0.1 \mathrm{~Hz}$. The multiplicity abbreviations used are: s singlet, d doublet, t triplet, q quartet, $\mathrm{m}$ multiplet.

GC-MS spectral data was recorded on an Agilent Technologies 7890A GC system with an Agilent 5975C VL MSD or an Agilent 5975C inert Mass Selective Detector (EI) and a HP5MS column. GC-FID spectral data was obtained on an Agilent Technologies 6890A equipped with a HP-5 quartz column $(0.32 \mathrm{~mm}$ x $30 \mathrm{~m}: 0.25 \mathrm{um})$ by flame ionization detection. Method used: Starting temperature $50{ }^{\circ} \mathrm{C}$, hold $3 \mathrm{~min}$, temperature increase $40^{\circ} \mathrm{C} / \mathrm{min}$, final temperature $280^{\circ} \mathrm{C}$, hold $3 \mathrm{~min}$. High-resolution mass spectra (HRMS) were measured by the MS service of the Organisch-Chemisches Institut, Westfälische WilhelmsUniversität Münster, using electrospray ionization (ESI) on a Bruker Daltonics, MicroToF spectrometer.

High-resolution mass spectra (HRMS) were obtained by the MS service of the OrganischChemisches Institut, Westfälische WilhelmsUniversität Münster, using electrospray ionisation (ESI) on a Bruker Daltonics, MicroToF spectrometer.

Thin layer chromatography was carried out on Merck silica gel $60 \mathrm{~F}_{254}$ pre-coated aluminium sheets and were visualized using UV light $(254 \mathrm{~nm})$ and stained with basic aqueous potassium permanganate. 


\subsection{Experimental Photochemistry}

Blue ( $3 \mathrm{~W}, \lambda_{\max }=420 \mathrm{~nm}$ ) LEDs were used for light irradiation (see figure S1).

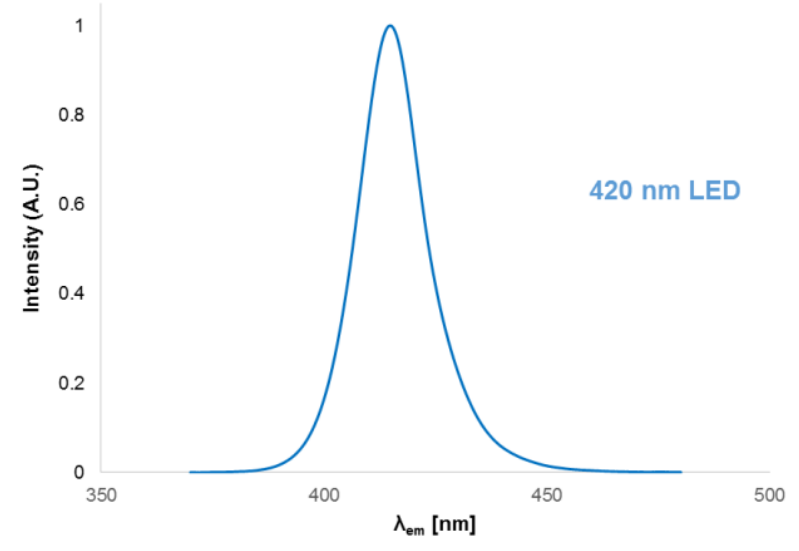

Figure S1. Emission spectra of the used LEDs.

In each case, the light source was placed $\sim 1 \mathrm{~cm}$ from the reaction vessel. A custom made "light box" was used with 6 LEDs arranged around a magnetic stirring plate (see figure S2). A fan attached to the apparatus was used to maintain the temperature inside the "box" at no more than $5{ }^{\circ} \mathrm{C}$ above room temperature.
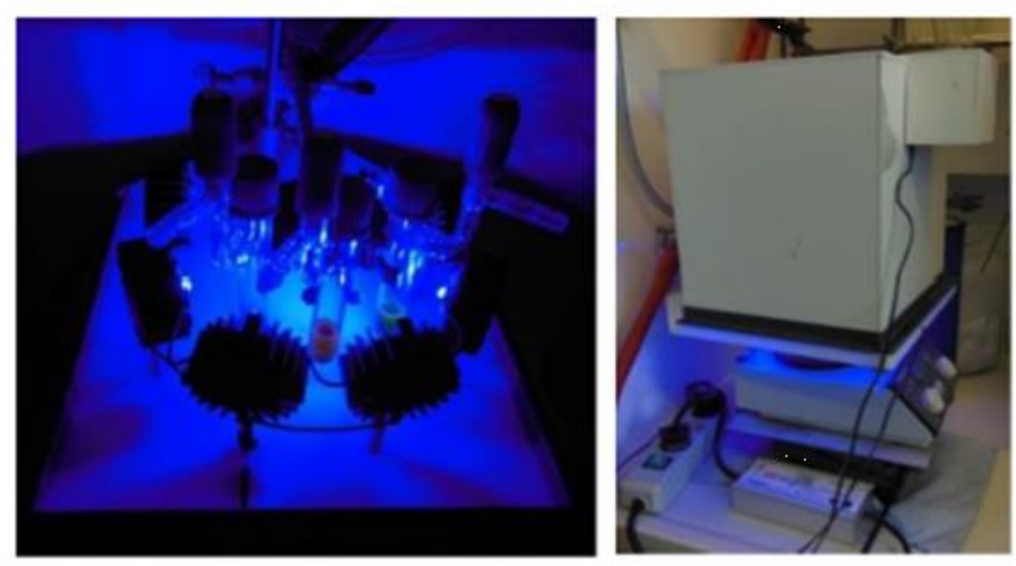

Figure S2. Photographs of the custom-made light box used for irradiation.

UV/Vis Absorption spectra were recorded on a JASCO V-730 two-beam UV/Vis spectrometer using the following parameter set: spectral bandwith $1.0 \mathrm{~nm}$, response time $0.06 \mathrm{~s}$, data interval $0.5 \mathrm{~nm}$, baseline correction. All samples were measured in Hellma fluorescence QS quartz cuvettes (chamber volume $1.4 \mathrm{~mL}$, dimension: $46 \mathrm{~mm}$ x $12.5 \mathrm{~mm}$ x $12.5 \mathrm{~mm}$ ) fitted with a PTFE stopper. 


\section{Experimental Procedures and Characterization Data}

\subsection{Synthesis of Acylsilanes}

Acylsilanes 1a-1c, 1e, 1f-1k, 1q-1r, 1t, 1z, and 2aa-2ac were known compounds, which prepared according to the literarures. ${ }^{[1]}$

\section{Preparation of 1d:}

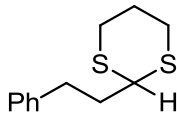

S1

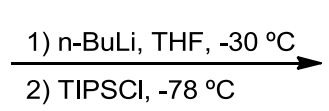

2) $\mathrm{TIPSCl},-78^{\circ} \mathrm{C}$

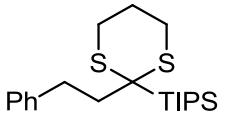

S2

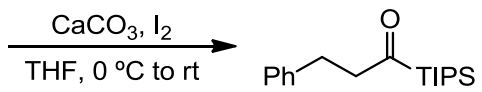

$1 \mathrm{~d}$

To a stirred solution of $\mathbf{S 1}(1.5 \mathrm{~g}, 6.68 \mathrm{mmol})$ in THF $(23.0 \mathrm{~mL})$ at $-30{ }^{\circ} \mathrm{C}$ was added $\mathrm{n}-\mathrm{BuLi}(5.01$ $\mathrm{mL}, 1.6 \mathrm{M}$ in hexanes, $8.02 \mathrm{mmol}$ ) dropwise. The resulting mixture was kept for $2 \mathrm{~h}$, then it was cooled to $-78{ }^{\circ} \mathrm{C}$, triisopropylsilyl chloride (TIPSCl, $1.55 \mathrm{~g}, 12.0 \mathrm{mmol}$ ) was added into the reaction mixture, and it was kept for another $1 \mathrm{~h}$ at $-78{ }^{\circ} \mathrm{C}$. Then the mixture was quenched with saturated aqueous $\mathrm{NH}_{4} \mathrm{Cl}(50 \mathrm{~mL})$, extracted with methyl tert-butyl ether $(50 \mathrm{~mL} \times 3)$. The combined organic phase was washed with brine $(100 \mathrm{~mL})$ and dried over $\mathrm{MgSO}_{4}$. The crude mixture was filtered through plug of silica using $\mathrm{CH}_{2} \mathrm{Cl}_{2}$ as an eluent afforded the $\mathbf{S 2}(2.24 \mathrm{~g}, 88 \%)$ as a colorless solid.

To a stirred solution of $\mathbf{S 2}(2.0 \mathrm{~g}, 5.25 \mathrm{mmol})$ in the combined solvent $\left(\mathrm{THF} / \mathrm{H}_{2} \mathrm{O}=4 / 1,35 \mathrm{~mL}\right)$ was added $\mathrm{CaCO}_{3}(4.2 \mathrm{~g}, 42 \mathrm{mmol})$ and $\mathrm{I}_{2}(8.08 \mathrm{~g}, 31.5 \mathrm{mmol})$ at $0{ }^{\circ} \mathrm{C}$. The mixture was kept for $1.5 \mathrm{~h}$ at room temperature, then quenched with saturated $\mathrm{Na}_{2} \mathrm{~S}_{2} \mathrm{O}_{3}(9 \mathrm{~mL})$. The mixture filtered through a pad of silica gel, washed by $\mathrm{CH}_{2} \mathrm{Cl}_{2}(15 \mathrm{~mL})$. Then the solution were washed with water $(20 \mathrm{~mL})$ and brine $\left(20 \mathrm{~mL}\right.$ ), dried over $\mathrm{MgSO}_{4}$, and concentrated. The residue was purified by flash chromatography to afford 1d $(1.4 \mathrm{~g}, 92 \%)$ as pale yellow oil. ${ }^{1} \mathbf{H}$ NMR $\left(300 \mathrm{MHz}, \mathrm{CDCl}_{3}\right) \delta 7.42-7.06(\mathrm{~m}, 1 \mathrm{H}), 3.04-$ $2.76(\mathrm{~m}, 1 \mathrm{H}), 1.41-1.23(\mathrm{~m}, 1 \mathrm{H}), 1.12(\mathrm{~d}, J=7.2 \mathrm{~Hz}, 4 \mathrm{H}) ;{ }^{13} \mathbf{C} \mathbf{N M R}\left(75 \mathrm{MHz}, \mathrm{CDCl}_{3}\right) \delta 246.08$, 141.87, 128.42, 128.38, 125.82, 53.07, 27.93, 18.54, 10.75; HRMS $\left(\mathrm{ESI}^{+}\right) \mathrm{m} / \mathrm{z}$ calcd. for $\mathrm{C}_{18} \mathrm{H}_{30} \mathrm{NaOSi}^{+}(\mathrm{M}+\mathrm{Na})^{+} 313.1958$, found 313.1967.

Preparation of 11, 1n, and 1y:
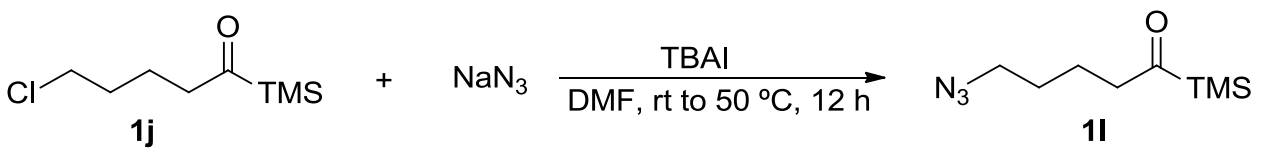

To a stirred solution of $\mathbf{1 j}$ ( $271 \mathrm{mg}, 1.4 \mathrm{mmol})$ in DMF $(9.0 \mathrm{~mL})$ was added tetrabutylammonium iodide $(21 \mathrm{mg}, 0.056 \mathrm{mmol})$ and sodium azide $(592 \mathrm{mg}, 9.1 \mathrm{mmol})$ at $\mathrm{rt}$. The resulting mixture was then warmed to $50{ }^{\circ} \mathrm{C}$ and stirred for $12 \mathrm{~h}$. Then the mixture was cooled to $\mathrm{rt}$ and quenched with $\mathrm{H}_{2} \mathrm{O}$ $(10 \mathrm{~mL})$, extracted with ethyl acetate $(15 \mathrm{~mL} \times 2)$. The combined organic phase was washed with brine $(10 \mathrm{~mL})$, dried over $\mathrm{Na}_{2} \mathrm{SO}_{4}$ and concentrated. The residue was purified by flash chromatography to afford 1n $(167 \mathrm{mg}, 60 \%)$ as white solid. ${ }^{1} \mathbf{H}$ NMR $\left(300 \mathrm{MHz}, \mathrm{CDCl}_{3}\right) \delta 3.26(\mathrm{t}, J=6.4 \mathrm{~Hz}, 2 \mathrm{H})$, $2.64(\mathrm{t}, J=6.7 \mathrm{~Hz}, 2 \mathrm{H}), 1.74-1.44(\mathrm{~m}, 4 \mathrm{H}), 0.20(\mathrm{~s}, 9 \mathrm{H}) ;{ }^{13} \mathrm{C}$ NMR $\left(75 \mathrm{MHz}, \mathrm{CDCl}_{3}\right) \delta 247.21$, 51.27, 47.47, 28.43, 19.21, -3.27; HRMS $\left(\mathrm{ESI}^{+}\right) \mathrm{m} / z$ calcd. for $\mathrm{C}_{8} \mathrm{H}_{17} \mathrm{~N}_{3} \mathrm{NaOSi}^{+}(\mathrm{M}+\mathrm{Na})^{+} 222.1033$, found 222.1033 .<smiles></smiles>
$1 k$<smiles>[Y]N1C(=O)c2ccccc2C1=O</smiles>

\section{DMF, rt, $6 \mathrm{~h}$}<smiles>CS(=O)(=O)CCCCN1C(=O)c2ccccc2C1=O</smiles> 
To a stirred solution of $\mathbf{1 k}(593 \mathrm{mg}, 2.5 \mathrm{mmol})$ in DMF $(5.0 \mathrm{~mL})$ was added phthalimide potassium salt $(602 \mathrm{mg}, 3.25 \mathrm{mmol})$. The resulting mixture was stirred for $6 \mathrm{~h}$ at $\mathrm{rt}$. Then the mixture was quenched with $\mathrm{H}_{2} \mathrm{O}(10 \mathrm{~mL})$, extracted with ethyl acetate $(15 \mathrm{~mL} \times 3)$. The combined organic phase was washed with brine $(10 \mathrm{~mL})$, dried over $\mathrm{Na}_{2} \mathrm{SO}_{4}$ and concentrated. The residue was purified by flash chromatography to afford $\mathbf{1 n}(493 \mathrm{mg}, 65 \%)$ as white solid. ${ }^{\mathbf{1}} \mathbf{H} \mathbf{~ N M R}\left(300 \mathrm{MHz}, \mathrm{CDCl}_{3}\right) \delta 7.92$ $-7.77(\mathrm{~m}, 2 \mathrm{H}), 7.77-7.60(\mathrm{~m}, 2 \mathrm{H}), 3.66(\mathrm{t}, J=6.9 \mathrm{~Hz}, 2 \mathrm{H}), 2.65(\mathrm{t}, J=7.0 \mathrm{~Hz}, 2 \mathrm{H}), 1.80-1.43(\mathrm{~m}$, 4H), $0.18(\mathrm{~s}, 9 \mathrm{H}) ;{ }^{13} \mathbf{C}$ NMR $\left(75 \mathrm{MHz}, \mathrm{CDCl}_{3}\right) \delta 247.62,168.40,133.90,132.10,123.19,47.53,37.64$, 28.07, 19.15, -3.22; HRMS (ESI+): calcd for $\mathrm{C}_{16} \mathrm{H}_{21} \mathrm{NNaO}_{3} \mathrm{Si}^{+}[\mathrm{M}+\mathrm{Na}]^{+} 326.1183$, found 326.1180.
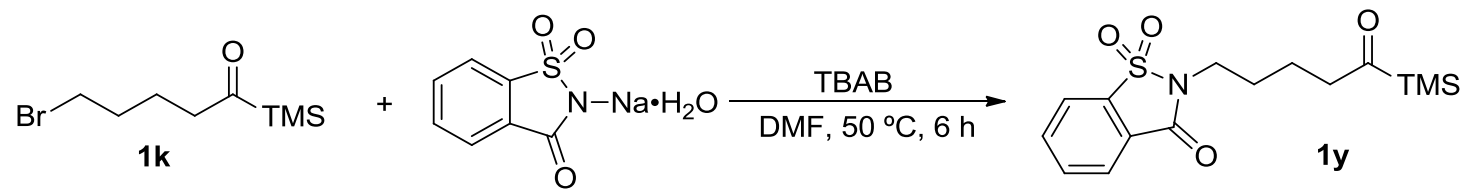

To a stirred solution of $\mathbf{1 k}$ ( $474 \mathrm{mg}, 2 \mathrm{mmol})$ in DMF $(5.0 \mathrm{~mL})$ was added tetrabutylammonium bromide (16 mg, $0.03 \mathrm{mmol})$ and saccharin sodium salt dihydrate $(349 \mathrm{mg}, 1.7 \mathrm{mmol})$ at $\mathrm{rt}$. The resulting mixture was stirred for $6 \mathrm{~h}$ at $50{ }^{\circ} \mathrm{C}$. Then the mixture was quenched with $\mathrm{H}_{2} \mathrm{O}(10 \mathrm{~mL})$, extracted with ethyl acetate $(15 \mathrm{~mL} \times 3)$. The combined organic phase was washed with brine $(10 \mathrm{~mL})$, dried over $\mathrm{Na}_{2} \mathrm{SO}_{4}$ and concentrated. The residue was purified by flash chromatography to afford $\mathbf{1 y}$ (288 mg, 50\%) as white solid. ${ }^{1} \mathbf{H}$ NMR $\left(400 \mathrm{MHz}, \mathrm{CDCl}_{3}\right) \delta 8.04(\mathrm{~d}, J=6.8 \mathrm{~Hz}, 1 \mathrm{H}), 7.94-7.73(\mathrm{~m}$, $3 \mathrm{H}), 3.75$ (t, $J=7.4 \mathrm{~Hz}, 2 \mathrm{H}), 2.67(\mathrm{t}, J=7.2 \mathrm{~Hz}, 2 \mathrm{H}), 1.81(\mathrm{dt}, J=15.2,7.5 \mathrm{~Hz}, 2 \mathrm{H}), 1.64$ (dd, $J=$ 15.1, $7.4 \mathrm{~Hz}, 2 \mathrm{H}), 0.18(\mathrm{~s}, 9 \mathrm{H}) ;{ }^{13} \mathbf{C}$ NMR $\left(101 \mathrm{MHz}, \mathrm{CDCl}_{3}\right) \delta 247.28,158.91,137.69,134.70$, 134.30, 127.40, 125.14, 120.90, 47.40, 39.09, 27.96, 19.14, -3.23; HRMS (ESI $\left.{ }^{+}\right) \mathrm{m} / \mathrm{z}$ calcd. for $\mathrm{C}_{15} \mathrm{H}_{22} \mathrm{NO}_{4} \mathrm{SSi}^{+}(\mathrm{M}+\mathrm{H})^{+} 340.1033$, found 340.1037.

\section{Preparation of 1m, 1s, and 1u-1x:}

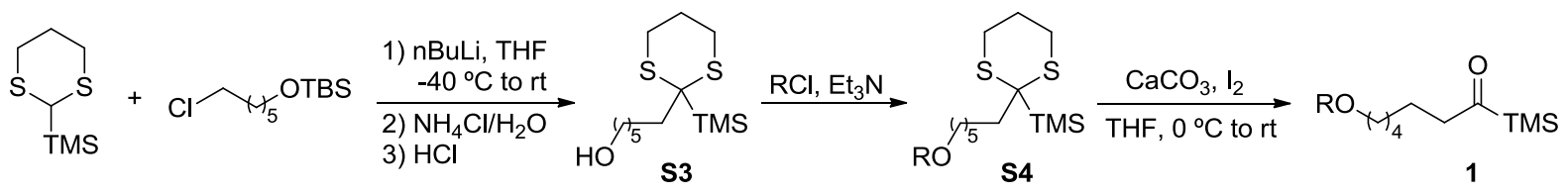

A solution of the 2-silyl dithiane $(6.0 \mathrm{mmol})$ in THF $(15 \mathrm{~mL})$ was treated with $\mathrm{n}$-BuLi $(6.3 \mathrm{mmol}$, 1.05 equiv.) at $\mathrm{rt}$ for $10 \mathrm{~min}$. The resulting anion solution was then cooled to $-40{ }^{\circ} \mathrm{C}$ and to this mixture a solution of 1-chloro-6-tert-butyldimethylsiloxyhexane $(6.6 \mathrm{mmol}, 1.1$ equiv.) in THF $(1 \mathrm{~mL})$ was added dropwise. The mixture was allowed to warm to rt while stirring for $2 \mathrm{~h}$, quenched with saturated aqueous $\mathrm{NH}_{4} \mathrm{Cl}$ and extracted with $\mathrm{Et}_{2} \mathrm{O}$. The combined organic layers were dried over $\mathrm{MgSO}_{4}$, filtered and concentrated. The resulting crude material was dissolved in EtOH $(15 \mathrm{~mL})$ and treated with conc. $\mathrm{HCl}(0.15 \mathrm{~mL})$ for $30 \mathrm{~min}$ at $\mathrm{rt}$. The reaction was then quenched with saturated aqueous $\mathrm{NaHCO}_{3}$ and extracted with $\mathrm{Et}_{2} \mathrm{O}$. The combined organic layers were washed with brine, dried over $\mathrm{Na}_{2} \mathrm{SO}_{4}$, filtered and concentrated. Flash chromatography (hexanes/EtOAc) provided the compound $\mathbf{S 3}$ in $87 \%$ yield as colorless oil.

To the mixture of $\mathbf{S 3}$ (4.0 mmol) and $\mathrm{NEt}_{3}\left(6 \mathrm{mmol}, 1.5\right.$ equiv.) in $\mathrm{CH}_{2} \mathrm{Cl}_{2}(30 \mathrm{~mL})$ was added acyl chloride (6 mmol, 1.5 equiv.) slowly at $0{ }^{\circ} \mathrm{C}$. The resulting solution was then warmed to $\mathrm{rt}$ and stirred overnight. The reaction mixture was quenched with $\mathrm{H}_{2} \mathrm{O}(10 \mathrm{~mL})$, then the layers were separated. The aqueous layer was extracted with EtOAc $(20 \mathrm{~mL} \times 3)$ and the combined organic layer was dried over $\mathrm{Na}_{2} \mathrm{SO}_{4}$ and then concentrated. Flash chromatography (hexanes/EtOAc) provided the compound $\mathbf{S 4}$. Then 1 could be obtained after the same procedure for the oxidative cleavage of the dithioketal moiety (preparation of 1d). 


\section{7-Oxo-7-(trimethylsilyl)heptyl 4-methylbenzenesulfonate (1m)}<smiles>CS(C)(C)C(=O)CCCCCCO[As]</smiles>

Following the general procedure, the title compound was obtained (799 mg, 56\% yield over two steps from S3, colorless oil). ${ }^{1} \mathbf{H}$ NMR $\left(300 \mathrm{MHz}, \mathrm{CDCl}_{3}\right) \delta 7.77(\mathrm{~d}, J=8.2 \mathrm{~Hz}, 2 \mathrm{H}), 7.34(\mathrm{~d}, J=8.1 \mathrm{~Hz}$, $2 \mathrm{H}), 4.00(\mathrm{t}, J=6.4 \mathrm{~Hz}, 2 \mathrm{H}), 2.55(\mathrm{t}, J=7.2 \mathrm{~Hz}, 2 \mathrm{H}), 2.44(\mathrm{~s}, 3 \mathrm{H}), 1.68-1.54(\mathrm{~m}, 2 \mathrm{H}), 1.53-1.37$ (m, 2H), $1.37-1.09$ (m, 4H), $0.18(\mathrm{~s}, 9 \mathrm{H}) ;{ }^{13} \mathbf{C}$ NMR $\left(75 \mathrm{MHz}, \mathrm{CDCl}_{3}\right) \delta$ 248.29, 144.68, 133.16, 129.82, 127.88, 70.54, 48.17, 28.64, 28.61, 25.27, 21.78, 21.65, -3.17; HRMS (ESI $\left.{ }^{+}\right) \mathrm{m} / z$ calcd. for $\mathrm{C}_{17} \mathrm{H}_{28} \mathrm{NaO}_{4} \mathrm{SSi}^{+}(\mathrm{M}+\mathrm{Na})^{+} 379.1370$, found 379.1370 .

\section{7-((tert-Butyldimethylsilyl)oxy)-1-(trimethylsilyl)heptan-1-one (1s)}

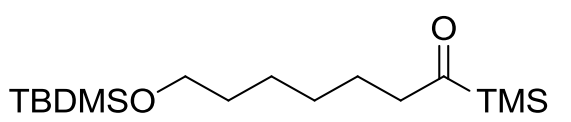

A solution of the 2-silyl dithiane $(6.0 \mathrm{mmol})$ in THF $(15 \mathrm{~mL})$ was treated with $\mathrm{n}-\mathrm{BuLi}$ ( $6.3 \mathrm{mmol}, 1.05$ equiv.) at $\mathrm{rt}$ for $10 \mathrm{~min}$. The resulting anion solution was then cooled to $-40{ }^{\circ} \mathrm{C}$ and to this mixture a solution of 1-chloro-6-tert-butyldimethylsiloxyhexane ( $6.6 \mathrm{mmol}, 1.1$ equiv.) in THF (1 $\mathrm{mL}$ ) was added dropwise. The mixture was allowed to warm to $\mathrm{rt}$ while stirring for $2 \mathrm{~h}$, quenched with saturated aqueous $\mathrm{NH}_{4} \mathrm{Cl}$ and extracted with $\mathrm{Et}_{2} \mathrm{O}$. The combined organic layers were dried over $\mathrm{MgSO}_{4}$, filtered and concentrated to afford the crude S4. After the same procedure for the oxidative cleavage of the dithioketal moiety (preparation of $\mathbf{1 d})$, the title compound $1 \mathrm{~s}$ was obtained $(1.36 \mathrm{~g}, 72 \%$ overall yield, colorless oil). ${ }^{1} \mathbf{H}$ NMR $\left(300 \mathrm{MHz}, \mathrm{CDCl}_{3}\right) \delta 3.58(\mathrm{t}, J=6.5 \mathrm{~Hz}, 2 \mathrm{H}), 2.58(\mathrm{t}, J=7.3 \mathrm{~Hz}$, 2H), $1.64-1.43(\mathrm{~m}, 4 \mathrm{H}), 1.38-1.19(\mathrm{~m}, 4 \mathrm{H}), 0.88(\mathrm{~s}, 9 \mathrm{H}), 0.18(\mathrm{~s}, 9 \mathrm{H}), 0.03(\mathrm{~s}, 6 \mathrm{H}) ;{ }^{13} \mathbf{C}$ NMR $(75$ $\left.\mathrm{MHz}, \mathrm{CDCl}_{3}\right) \delta 248.35,63.13,48.38,32.68,29.14,25.96,25.67,22.14,18.34,-3.16,-5.29 ;$ HRMS $\left(\mathrm{ESI}^{+}\right) \mathrm{m} / \mathrm{z}$ calcd. for $\mathrm{C}_{16} \mathrm{H}_{37} \mathrm{O}_{2} \mathrm{Si}_{2}{ }^{+}(\mathrm{M}+\mathrm{H})^{+}$317.2327, found 317.2327.

\section{7-Oxo-7-(trimethylsilyl)heptyl benzoate (1u)}

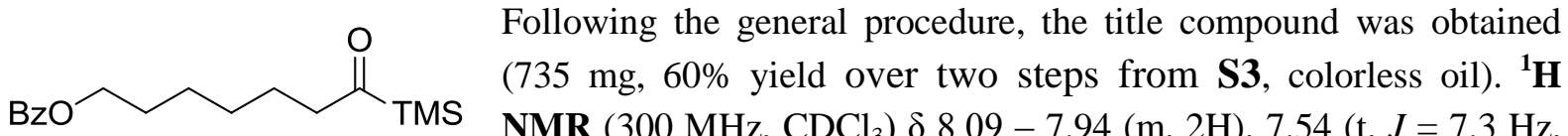
$1 \mathrm{H}), 7.42(\mathrm{t}, J=7.6 \mathrm{~Hz}, 2 \mathrm{H}), 4.29(\mathrm{t}, J=6.6 \mathrm{~Hz}, 2 \mathrm{H}), 2.60(\mathrm{t}, J=7.2 \mathrm{~Hz}, 2 \mathrm{H}), 1.85-1.64(\mathrm{~m}, 2 \mathrm{H})$, $1.61-1.19(\mathrm{~m}, 6 \mathrm{H}), 0.18(\mathrm{~s}, 9 \mathrm{H}) ;{ }^{13} \mathbf{C}$ NMR $\left(75 \mathrm{MHz}, \mathrm{CDCl}_{3}\right) \delta 248.40,166.66,132.82,130.46$, 129.53, 128.33, 64.95, 48.30, 28.97, 28.57, 25.95, 21.96, -3.16; HRMS (ESI $\left.{ }^{+}\right) \mathrm{m} / \mathrm{z}$ calcd. for $\mathrm{C}_{17} \mathrm{H}_{26} \mathrm{NaO}_{3} \mathrm{Si}^{+}(\mathrm{M}+\mathrm{Na})^{+} 329.1543$, found 329.1543 .

\section{7-Oxo-7-(trimethylsilyl)heptyl pivalate (1v)}

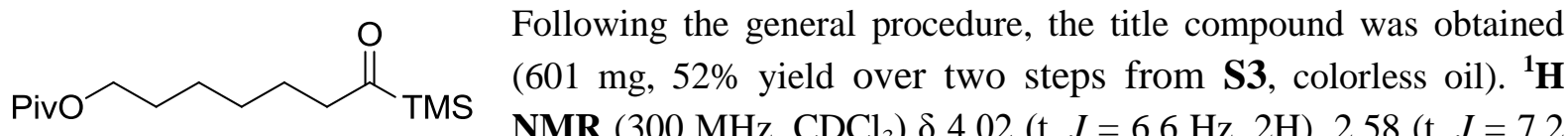

$\mathrm{Hz}, 2 \mathrm{H}), 1.57(\mathrm{~m}, 4 \mathrm{H}), 1.42-1.23(\mathrm{~m}, 4 \mathrm{H}), 1.17(\mathrm{~s}, 9 \mathrm{H}), 0.18(\mathrm{~s}, 9 \mathrm{H}) ;{ }^{13} \mathbf{C} \mathbf{N M R}\left(75 \mathrm{MHz}, \mathrm{CDCl}_{3}\right) \delta$ 248.38, 178.62, 64.27, 48.30, 38.72, 28.91, 28.44, 27.19, 25.80, 21.93, -3.17; HRMS (ESI $\left.{ }^{+}\right) \mathrm{m} / z$ calcd. for $\mathrm{C}_{15} \mathrm{H}_{30} \mathrm{NaO}_{3} \mathrm{Si}^{+}(\mathrm{M}+\mathrm{Na})^{+}$309.1856, found 309.1856 .

\section{7-Oxo-7-(trimethylsilyl)heptyl furan-2-carboxylate (1w)}<smiles>CS(=O)(=O)CCCCCCOC(=O)c1ccco1</smiles>

Following the general procedure, the title compound was obtained $(652 \mathrm{mg}, 55 \%$ yield over two steps from S3, colorless oil). ${ }^{1} \mathbf{H}$ NMR $\left(300 \mathrm{MHz}, \mathrm{CDCl}_{3}\right) \delta 7.77(\mathrm{dd}, J=3.7$, $0.7 \mathrm{~Hz}, 1 \mathrm{H}), 7.53(\mathrm{dd}, J=5.0,0.7 \mathrm{~Hz}, 1 \mathrm{H}), 7.08(\mathrm{dd}, J=4.7$, $4.0 \mathrm{~Hz}, 1 \mathrm{H}), 4.26(\mathrm{t}, J=6.6 \mathrm{~Hz}, 2 \mathrm{H}), 2.59(\mathrm{t}, J=7.2 \mathrm{~Hz}, 2 \mathrm{H}), 1.79-1.66(\mathrm{~m}, 2 \mathrm{H}), 1.60-1.18(\mathrm{~m}, 6 \mathrm{H})$, $0.18(\mathrm{~s}, 9 \mathrm{H}) ;{ }^{13} \mathbf{C}$ NMR $\left(75 \mathrm{MHz}, \mathrm{CDCl}_{3}\right) \delta 248.40,162.31,134.04,133.26,132.20,127.71,65.11$, 
$48.29,28.92,28.52,25.85,21.93,-3.16$; $\mathbf{H R M S}\left(\mathrm{ESI}^{+}\right) \mathrm{m} / \mathrm{z}$ calcd. for $\mathrm{C}_{15} \mathrm{H}_{24} \mathrm{NaO}_{4} \mathrm{Si}^{+}(\mathrm{M}+\mathrm{Na})^{+}$ 319.1336, found 319.1342 .

\section{7-Oxo-7-(trimethylsilyl)heptyl thiophene-2-carboxylate (1x)}<smiles>CS(=O)(=O)CCCCCCOC(=O)c1cccs1</smiles>

Following the general procedure, the title compound was obtained (625 mg, 50\% yield over two steps from $\mathbf{S 3}$,

colorless oil). ${ }^{1} \mathbf{H}$ NMR $\left(300 \mathrm{MHz}, \mathrm{CDCl}_{3}\right) \delta 7.56(\mathrm{~d}, J=0.8$ $\mathrm{Hz}, 1 \mathrm{H}), 7.15(\mathrm{~d}, J=3.5 \mathrm{~Hz}, 1 \mathrm{H}), 6.49(\mathrm{dd}, J=3.4,1.7 \mathrm{~Hz}$, $1 \mathrm{H}), 4.28(\mathrm{t}, J=6.7 \mathrm{~Hz}, 2 \mathrm{H}), 2.59(\mathrm{t}, J=7.2 \mathrm{~Hz}, 2 \mathrm{H}), 1.87-1.64(\mathrm{~m}, 2 \mathrm{H}), 1.61-1.18(\mathrm{~m}, 7 \mathrm{H}), 0.18$ $(\mathrm{s}, 9 \mathrm{H}) ;{ }^{13} \mathrm{C}$ NMR $\left(75 \mathrm{MHz}, \mathrm{CDCl}_{3}\right) \delta 248.41,158.84,146.20,144.83,117.73,111.79,64.92,48.28$, 28.92, 28.53, 25.80, 21.93, -3.16.; HRMS $\left(\mathrm{ESI}^{+}\right) \mathrm{m} / z$ calcd. for $\mathrm{C}_{15} \mathrm{H}_{24} \mathrm{NaO}_{3} \mathrm{SSi}^{+}(\mathrm{M}+\mathrm{Na})^{+} 335.1108$, found 335.1110 .

\section{Preparation of 1o:}

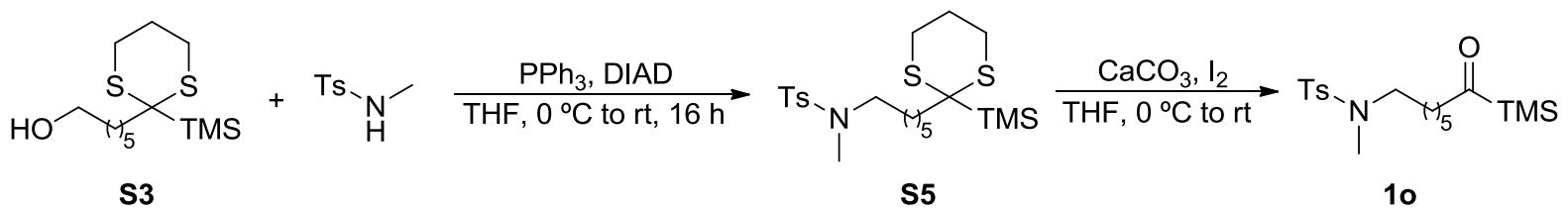

To a solution of $\mathbf{S 3}$ ( $878 \mathrm{mg}, 3 \mathrm{mmol}), N$-methyl- $p$-toluenesulfonamide (556 mg, $3 \mathrm{mmol}$ ), and triphenylphosphine $(865 \mathrm{mg}, 3.3 \mathrm{mmol})$ in THF $(3 \mathrm{~mL})$ cooled in an ice-water bath was added diisopropyl azodicarboxylate $(649 \mathrm{uL}, 3.3 \mathrm{mmol})$ dropwise under argon. The reaction mixture was warm to $\mathrm{rt}$ and stirred for $16 \mathrm{~h}$. Then the reaction mixture was concentrated and chromatographed on silica gel (hexane/EtOAc, 85:15) to give $\mathbf{S 5}$ (1.000 g, 75\% yield) as colorless oil.

To a stirred solution of $\mathbf{S 5}(2.25 \mathrm{mmol})$ in the combined solvent $\left(\mathrm{THF} / \mathrm{H}_{2} \mathrm{O}=4 / 1,15 \mathrm{~mL}\right)$ was added $\mathrm{CaCO}_{3}(1.8 \mathrm{~g}, 18 \mathrm{mmol})$ and $\mathrm{I}_{2}(3.45 \mathrm{~g}, 13.5 \mathrm{mmol})$ at $0{ }^{\circ} \mathrm{C}$. The mixture was kept for $1.5 \mathrm{~h}$ at room temperature, then quenched with saturated $\mathrm{Na}_{2} \mathrm{~S}_{2} \mathrm{O}_{3}(5 \mathrm{~mL})$. The mixture filtered through a pad of silica gel, washed by $\mathrm{CH}_{2} \mathrm{Cl}_{2}(15 \mathrm{~mL})$. Then the solution were washed with water $(20 \mathrm{~mL})$ and brine $(20 \mathrm{~mL})$, dried over $\mathrm{MgSO}_{4}$, and concentrated. The residue was purified by flash chromatography to afford $10(624 \mathrm{mg}, 75 \%)$ as colorless oil. ${ }^{1} \mathbf{H}$ NMR $\left(400 \mathrm{MHz}, \mathrm{CDCl}_{3}\right) \delta 7.62(\mathrm{~d}, J=8.3 \mathrm{~Hz}, 2 \mathrm{H}), 7.28$ $(\mathrm{d}, J=7.9 \mathrm{~Hz}, 2 \mathrm{H}), 2.99-2.84(\mathrm{~m}, 2 \mathrm{H}), 2.66(\mathrm{~s}, 3 \mathrm{H}), 2.57$ (t, $J=7.3 \mathrm{~Hz}, 2 \mathrm{H}), 2.39$ (s, 3H), $1.56-$ $1.39(\mathrm{~m}, 4 \mathrm{H}), 1.36-1.14(\mathrm{~m}, 4 \mathrm{H}), 0.17(\mathrm{~s}, 9 \mathrm{H}) ;{ }^{13} \mathbf{C ~ N M R}\left(101 \mathrm{MHz}, \mathrm{CDCl}_{3}\right) \delta 246.54,141.24$, 132.60, 127.67, 125.44, 48.03, 46.37, 32.61, 26.89, 25.41, 24.38, 19.96, 19.54, -5.10; HRMS (ESI $\left.{ }^{+}\right)$ $m / z$ calcd. for $\mathrm{C}_{18} \mathrm{H}_{31} \mathrm{NNaO}_{3} \mathrm{SSi}^{+}(\mathrm{M}+\mathrm{Na})^{+}$392.1686, found 392.1686.

\section{Preparation of 1p:}

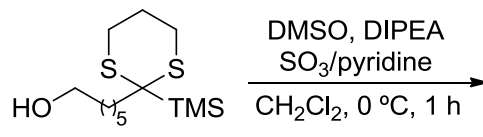

S3

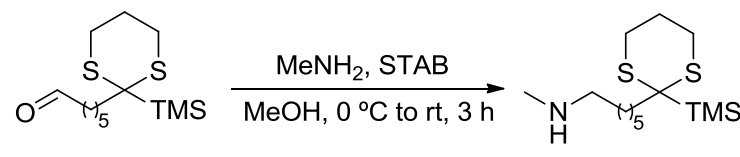

S6
S7

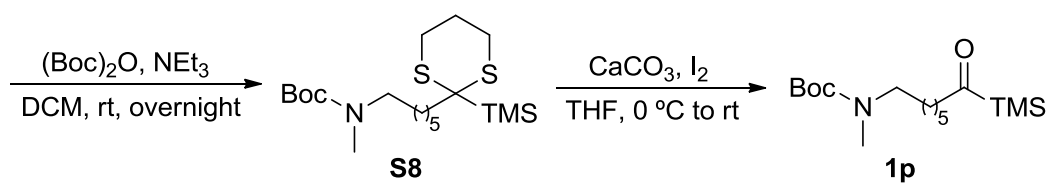

At $0{ }^{\circ} \mathrm{C}$, a solution of $\mathbf{S 3}(5.0 \mathrm{mmol})$, DMSO $(25.0 \mathrm{mmol})$ and DIPEA $(15.0 \mathrm{mmol})$ in $\mathrm{CH}_{2} \mathrm{Cl}_{2}(15 \mathrm{~mL})$ was treated with $\mathrm{SO}_{3} /$ pyridine $(15.0 \mathrm{mmol})$. After $60 \mathrm{~min}$, the mixture was diluted with $\mathrm{Et}_{2} \mathrm{O}$, washed 
(water, $\mathrm{NaHSO}_{4}$, water, brine), dried $\left(\mathrm{Na}_{2} \mathrm{SO}_{4}\right)$, filtered, and concentrated. Flash chromatography (3\% ethyl acetate/hexanes) afforded aldehyde $\mathbf{S 6}(1.16 \mathrm{~g}, 80 \%)$ as colorless oil.

To a methylamine solution $33 \mathrm{wt} . \%$ in absolute ethanol was added aldehyde $\mathbf{S 6}(1 \mathrm{~g}, 3.4 \mathrm{mmol})$ at $0{ }^{\circ} \mathrm{C}$. After $10 \mathrm{~min}$, sodium triacetoxyborohydride $(1.45 \mathrm{~g}, 6.83 \mathrm{mmol})$ was added potionwise. The resulting mixture was warm to $\mathrm{rt}$ and stirred for $3 \mathrm{~h}$. Then the reaction was quenched by water $(5 \mathrm{~mL})$, extracted by ethyl acetate $(10 \mathrm{~mL} \times 3)$ and the combined organic layer was dried over $\mathrm{Na}_{2} \mathrm{SO}_{4}$ and then concentrated to afford the crude product $\mathbf{S 7}$, which was directly used for the next step without further purification. To a solution of $\mathbf{S 7}$ in DCM (10 mL) was added $\mathrm{NEt}_{3}(476 \mathrm{uL}, 3.4 \mathrm{mmol})$ and $(\mathrm{Boc})_{2} \mathrm{O}$ (941 uL, $4.1 \mathrm{mmol}$ ). The resulting mixture was stirred overnight and then concentrated. Purification by

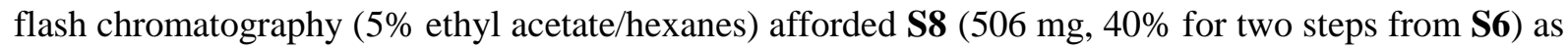
colorless oil.

To a stirred solution of $\mathbf{S 8}(1.25 \mathrm{mmol})$ in the combined solvent $\left(\mathrm{THF} / \mathrm{H}_{2} \mathrm{O}=4 / 1,10 \mathrm{~mL}\right)$ was added $\mathrm{CaCO}_{3}(1 \mathrm{~g}, 10 \mathrm{mmol})$ and $\mathrm{I}_{2}(1.9 \mathrm{~g}, 7.5 \mathrm{mmol})$ at $0{ }^{\circ} \mathrm{C}$. The mixture was kept for $1.5 \mathrm{~h}$ at room temperature, then quenched with saturated $\mathrm{Na}_{2} \mathrm{~S}_{2} \mathrm{O}_{3}(5 \mathrm{~mL})$. The mixture filtered through a pad of silica gel, washed by $\mathrm{CH}_{2} \mathrm{Cl}_{2}(15 \mathrm{~mL})$. Then the solution were washed with water $(20 \mathrm{~mL})$ and brine $\left(20 \mathrm{~mL}\right.$ ), dried over $\mathrm{MgSO}_{4}$, and concentrated. The residue was purified by flash chromatography to afford 1p (308 mg, 78\%) as colorless oil. ${ }^{1} \mathbf{H}$ NMR $\left(300 \mathrm{MHz}, \mathrm{CDCl}_{3}\right) \delta 3.17(\mathrm{t}, J=7.2 \mathrm{~Hz}, 2 \mathrm{H}), 2.81$ $(\mathrm{s}, 3 \mathrm{H}), 2.58(\mathrm{t}, J=7.3 \mathrm{~Hz}, 2 \mathrm{H}), 1.60-1.39(\mathrm{~m}, 13 \mathrm{H}), 1.37-1.16(\mathrm{~m}, 4 \mathrm{H}), 0.18(\mathrm{~s}, 9 \mathrm{H}) ;{ }^{13} \mathbf{C} \mathbf{~ N M R}$ $\left(75 \mathrm{MHz}, \mathrm{CDCl}_{3}\right) \delta 248.17,155.75,78.99,48.55,48.26,33.98,29.04,28.43,27.57,26.53,22.02$, 3.21; HRMS $\left(\mathrm{ESI}^{+}\right) \mathrm{m} / z$ calcd. for $\mathrm{C}_{16} \mathrm{H}_{34} \mathrm{NO}_{3} \mathrm{Si}^{+}(\mathrm{M}+\mathrm{H})^{+} 316.2302$, found 316.2314 .

\subsection{Substrate Scope}

\section{General Procedure}

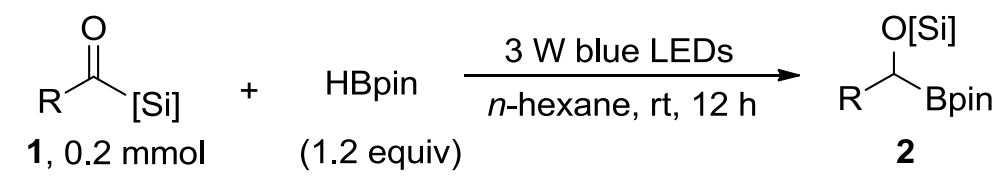

To an oven-dried screw-cap $5 \mathrm{~mL}$ reaction tube was charged the acylsilane substrate (if solid, 0.2 mmol, 1.0 equiv.). The tube was then evacuated and back-filled with argon for 3 times. Subsequently, $n$-hexane $(2 \mathrm{~mL})$ was added followed by acylsilane substrate (if liquid, $0.2 \mathrm{mmol}, 1.0$ equiv.) and HBpin (0.24 mmol, 1.2 equiv.) under argon. Once added, the tube was closed and the reaction mixture was stirred and irradiated in a photobox with blue LEDs $(\lambda \max =420 \mathrm{~nm})$ for $12 \mathrm{~h}$. The mixture was concentrated to directly afford the product without column chromatography (excess of HBpin was removed in vacuo).

\section{Trimethyl(3-phenyl-1-(4,4,5,5-tetramethyl-1,3,2-dioxaborolan-2-yl)propoxy)silane (2a)}<smiles>COC(CCc1ccccc1)Cc1ccccc1</smiles>

Following the general procedure, the title compound was obtained $(75 \mathrm{mg}$, quant., colorless oil). ${ }^{1} \mathbf{H}$ NMR (400 MHz, $\left.\mathrm{CD}_{2} \mathrm{Cl}_{2}\right) \delta 7.39-7.09(\mathrm{~m}, 5 \mathrm{H}), 3.54$ $(\mathrm{t}, J=6.6 \mathrm{~Hz}, 1 \mathrm{H}), 2.69(\mathrm{dtd}, J=22.0,13.8,8.1 \mathrm{~Hz}, 2 \mathrm{H}), 1.84(\mathrm{dd}, J=15.6$, $7.3 \mathrm{~Hz}, 2 \mathrm{H}), 1.25(\mathrm{~d}, J=3.3 \mathrm{~Hz}, 13 \mathrm{H}), 0.12(\mathrm{~s}, 9 \mathrm{H}) ;{ }^{13} \mathbf{C}$ NMR $(101 \mathrm{MHz}$, $\left.\mathrm{CD}_{2} \mathrm{Cl}_{2}\right) \delta 144.02,129.65,129.43,126.79,84.88,37.62,34.07,25.98,25.62,0.90$ (The carbon attached to boron was not observed due to quadrupolar relaxation); HRMS (ESI $\left.{ }^{+}\right) \mathrm{m} / \mathrm{z}$ calcd. for $\mathrm{C}_{18} \mathrm{H}_{31} \mathrm{BNaO}_{3} \mathrm{Si}^{+}(\mathrm{M}+\mathrm{Na})^{+}$357.2028, found 357.2039. 
Triethyl(3-phenyl-1-(4,4,5,5-tetramethyl-1,3,2-dioxaborolan-2-yl)propoxy)silane (2b)<smiles>OS(=S)(=S)CCCc1ccccc1</smiles>

Following the general procedure, the title compound was obtained $(75 \mathrm{mg}$, quant., colorless oil). ${ }^{1} \mathbf{H}$ NMR $\left(400 \mathrm{MHz}, \mathrm{CD}_{2} \mathrm{Cl}_{2}\right) \delta 7.28-6.94(\mathrm{~m}, 5 \mathrm{H}), 3.49$ $(\mathrm{t}, J=6.5 \mathrm{~Hz}, 1 \mathrm{H}), 2.71-2.49(\mathrm{~m}, 2 \mathrm{H}), 1.84-1.65(\mathrm{~m}, 2 \mathrm{H}), 1.16(\mathrm{~d}, J=3.1$ $\mathrm{Hz}, 12 \mathrm{H}), 0.89(\mathrm{t}, J=7.9 \mathrm{~Hz}, 9 \mathrm{H}), 0.53(\mathrm{t}, J=7.6 \mathrm{~Hz}, 6 \mathrm{H}) ;{ }^{13} \mathbf{C}$ NMR $(101$ $\left.\mathrm{MHz}, \mathrm{CD}_{2} \mathrm{Cl}_{2}\right) \delta 142.91,128.37,128.20,125.53,83.64,36.91,32.77,24.72,24.41,6.70,4.65$ (The carbon attached to boron was not observed due to quadrupolar relaxation); HRMS (ESI $\left.{ }^{+}\right) \mathrm{m} / z$ calcd. for $\mathrm{C}_{21} \mathrm{H}_{37} \mathrm{BNaO}_{3} \mathrm{Si}^{+}(\mathrm{M}+\mathrm{Na})^{+}$399.2497, found 399.2501.

tert-Butyldimethyl(3-phenyl-1-(4,4,5,5-tetramethyl-1,3,2-dioxaborolan-2-yl)propoxy)silane (2c)<smiles>O=[SH](O)(O)c1ccccc1</smiles>

Following the general procedure, the title compound was obtained $(75 \mathrm{mg}$, quant., colorless oil). ${ }^{1} \mathbf{H}$ NMR $\left(300 \mathrm{MHz}, \mathrm{CD}_{2} \mathrm{Cl}_{2}\right) \delta 7.37-7.17(\mathrm{~m}, 5 \mathrm{H})$, $3.63(\mathrm{t}, J=6.7 \mathrm{~Hz}, 1 \mathrm{H}), 2.96-2.54(\mathrm{~m}, 2 \mathrm{H}), 1.89(\mathrm{dd}, J=15.5,7.7 \mathrm{~Hz}, 2 \mathrm{H})$, $1.30(\mathrm{~d}, J=2.9 \mathrm{~Hz}, 12 \mathrm{H}), 0.98(\mathrm{~s}, 9 \mathrm{H}), 0.12(\mathrm{~s}, 6 \mathrm{H}) ;{ }^{13} \mathbf{C} \mathbf{N M R}(75 \mathrm{MHz}$, $\left.\mathrm{CD}_{2} \mathrm{Cl}_{2}\right) \delta 142.92,128.36,128.18,125.52,83.61,36.80,32.93,25.76,24.79$,

$24.38,18.36,-5.15,-5.21$ (The carbon attached to boron was not observed due to quadrupolar relaxation); HRMS $\left(\mathrm{ESI}^{+}\right) \mathrm{m} / z$ calcd. for $\mathrm{C}_{21} \mathrm{H}_{37} \mathrm{BNaO}_{3} \mathrm{Si}^{+}(\mathrm{M}+\mathrm{Na})^{+} 399.2497$, found 399.2501

Triisopropyl(3-phenyl-1-(4,4,5,5-tetramethyl-1,3,2-dioxaborolan-2-yl)propoxy)silane (2d)

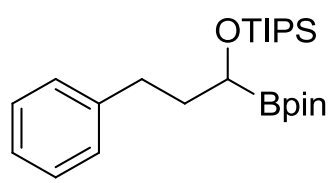

Following the general procedure, the title compound was obtained $(84 \mathrm{mg}$, quant., colorless oil). ${ }^{1} \mathbf{H}$ NMR $\left(400 \mathrm{MHz}, \mathrm{CD}_{2} \mathrm{Cl}_{2}\right) \delta 7.36-6.88(\mathrm{~m}, 5 \mathrm{H}), 3.66$ $(\mathrm{dd}, J=7.0,5.4 \mathrm{~Hz}, 1 \mathrm{H}), 2.77-2.45(\mathrm{~m}, 2 \mathrm{H}), 1.96-1.71(\mathrm{~m}, 2 \mathrm{H}), 1.18(\mathrm{~d}, J=$ $3.0 \mathrm{~Hz}, 12 \mathrm{H}), 1.03-0.93(\mathrm{~m}, 21 \mathrm{H}) ;{ }^{13} \mathbf{C}$ NMR $\left(101 \mathrm{MHz}, \mathrm{CD}_{2} \mathrm{Cl}_{2}\right) \delta 142.97$, $128.35,128.20,125.54,83.65,37.51,32.43,24.84,24.49,17.95,17.89,12.32$ (The carbon attached to boron was not observed due to quadrupolar relaxation); HRMS (ESI $\left.{ }^{+}\right) \mathrm{m} / z$ calcd. for $\mathrm{C}_{24} \mathrm{H}_{43} \mathrm{BNaO}_{3} \mathrm{Si}^{+}(\mathrm{M}+\mathrm{Na})^{+}$441.2967, found 441.2971.

Dimethyl(phenyl)(3-phenyl-1-(4,4,5,5-tetramethyl-1,3,2-dioxaborolan-2-yl)propoxy)silane (2e)<smiles>COC(CCc1ccccc1)Cc1ccccc1</smiles>

Following the general procedure, the title compound was obtained (79 $\mathrm{mg}$, quant., colorless oil). ${ }^{1} \mathbf{H}$ NMR $\left(300 \mathrm{MHz}, \mathrm{CD}_{2} \mathrm{Cl}_{2}\right) \delta 7.62-7.53(\mathrm{~m}, 2 \mathrm{H})$, $7.29(\mathrm{tt}, J=4.2,2.6 \mathrm{~Hz}, 3 \mathrm{H}), 7.23-7.13(\mathrm{~m}, 2 \mathrm{H}), 7.08(\mathrm{t}, J=6.3 \mathrm{~Hz}, 3 \mathrm{H})$, $3.51(\mathrm{t}, J=6.5 \mathrm{~Hz}, 1 \mathrm{H}), 2.78-2.44(\mathrm{~m}, 2 \mathrm{H}), 1.77(\mathrm{ddd}, J=12.7,7.8,1.1 \mathrm{~Hz}$, $2 \mathrm{H}), 1.15(\mathrm{~s}, 12 \mathrm{H}), 0.31(\mathrm{~s}, 6 \mathrm{H}) ;{ }^{13} \mathbf{C}$ NMR $\left(101 \mathrm{MHz}, \mathrm{CD}_{2} \mathrm{Cl}_{2}\right) \delta 142.74$,

138.61, 133.66, 129.35, 128.39, 128.19, 127.63, 125.54, 83.73, 36.45, 32.76, 24.71, 24.40, -1.64, -1.66 (The carbon attached to boron was not observed due to quadrupolar relaxation); HRMS $\left(\mathrm{ESI}^{+}\right) \mathrm{m} / \mathrm{z}$ calcd. for $\mathrm{C}_{23} \mathrm{H}_{33} \mathrm{BNaO}_{3} \mathrm{Si}^{+}(\mathrm{M}+\mathrm{Na})^{+} 419.2184$, found 419.2189 .

Trimethyl((1-(4,4,5,5-tetramethyl-1,3,2-dioxaborolan-2-yl)decyl)oxy)silane (2f)

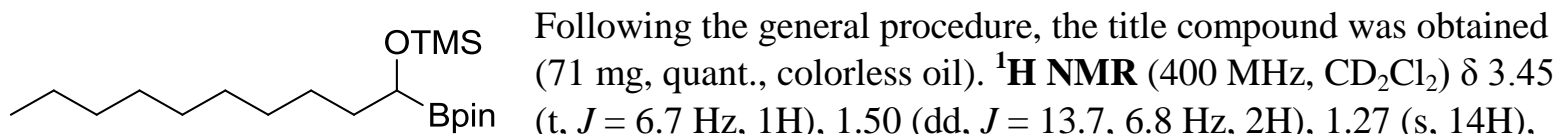
$1.23(\mathrm{~d}, J=3.3 \mathrm{~Hz}, 12 \mathrm{H}), 0.88(\mathrm{t}, J=6.9 \mathrm{~Hz}, 3 \mathrm{H}), 0.07(\mathrm{~s}, 9 \mathrm{H}) .{ }^{13} \mathbf{C} \mathbf{~ N M R}\left(101 \mathrm{MHz}, \mathrm{CD}_{2} \mathrm{Cl}_{2}\right) \delta 84.81$, $35.66,33.25,30.99,30.92,30.90,30.65,27.65,26.00,25.71,24.01,15.20,0.90$ (The carbon attached to boron was not observed due to quadrupolar relaxation); $\mathbf{H R M S}\left(\mathrm{ESI}^{+}\right) \mathrm{m} / z$ calcd. for $\mathrm{C}_{19} \mathrm{H}_{42} \mathrm{BO}_{3} \mathrm{Si}^{+}$ $(\mathrm{M}+\mathrm{H})^{+}$357.2991, found 357.2998.

(Cyclohexyl(4,4,5,5-tetramethyl-1,3,2-dioxaborolan-2-yl)methoxy)trimethylsilane (2g)<smiles>CS(=O)(=O)OC(Br)C1CCCCC1</smiles> 
Following the general procedure, the title compound was obtained (62 mg, quant., colorless oil). ${ }^{1} \mathbf{H}$ NMR $\left(400 \mathrm{MHz}, \mathrm{CD}_{2} \mathrm{Cl}_{2}\right) \delta 3.21(\mathrm{~d}, J=6.2 \mathrm{~Hz}, 1 \mathrm{H}), 1.80-1.55(\mathrm{~m}, 6 \mathrm{H}), 1.55-1.39(\mathrm{~m}, 1 \mathrm{H}), 1.23$ $(\mathrm{d}, J=3.5 \mathrm{~Hz}, 12 \mathrm{H}), 1.20-0.96(\mathrm{~m}, 4 \mathrm{H}), 0.06(\mathrm{~s}, 9 \mathrm{H}) ;{ }^{13} \mathbf{C} \mathbf{N M R}\left(101 \mathrm{MHz}, \mathrm{CD}_{2} \mathrm{Cl}_{2}\right) \delta 84.76,43.39$, $31.31,30.98,27.91,27.74,27.73,26.09,25.77,0.90$ (The carbon attached to boron was not observed due to quadrupolar relaxation); HRMS (ESI $\left.{ }^{+}\right) m / z$ calcd. for $\mathrm{C}_{16} \mathrm{H}_{34} \mathrm{BO}_{3} \mathrm{Si}^{+}(\mathrm{M}+\mathrm{H})^{+} 313.2365$, found 313.2372 .

Trimethyl((1-(4,4,5,5-tetramethyl-1,3,2-dioxaborolan-2-yl)undec-10-en-1-yl)oxy)silane (2i)

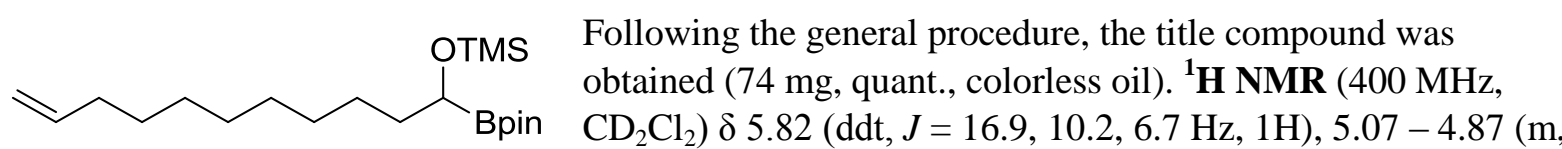
$2 \mathrm{H}), 3.45(\mathrm{t}, J=6.7 \mathrm{~Hz}, 1 \mathrm{H}), 2.11-1.96(\mathrm{~m}, 2 \mathrm{H}), 1.56-1.45(\mathrm{~m}, 2 \mathrm{H}), 1.41-1.26(\mathrm{~m}, 12 \mathrm{H}), 1.23(\mathrm{~d}$, $J=3.4 \mathrm{~Hz}, 12 \mathrm{H}), 0.07(\mathrm{~s}, 9 \mathrm{H}) ;{ }^{13} \mathbf{C} \mathbf{N M R}\left(101 \mathrm{MHz}, \mathrm{CD}_{2} \mathrm{Cl}_{2}\right) \delta 140.64,115.10,84.81,35.64,35.13$, $30.96,30.85,30.75,30.46,30.30,27.64,26.00,25.71,0.90$ (The carbon attached to boron was not observed due to quadrupolar relaxation); $\mathbf{H R M S}\left(\mathrm{ESI}^{+}\right) \mathrm{m} / z$ calcd. for $\mathrm{C}_{20} \mathrm{H}_{41} \mathrm{BNaO}_{3} \mathrm{Si}^{+}(\mathrm{M}+\mathrm{Na})^{+}$ 391.2810, found 391.2814.

((5-Chloro-1-(4,4,5,5-tetramethyl-1,3,2-dioxaborolan-2-yl)pentyl)oxy)trimethylsilane (2j)

Following the general procedure, the title compound was obtained $(64 \mathrm{mg}$,
Quant., colorless oil). ${ }^{1} \mathbf{H}$ NMR $\left(300 \mathrm{MHz}, \mathrm{CD}_{2} \mathrm{Cl}_{2}\right) \delta 3.54(\mathrm{t}, J=6.8 \mathrm{~Hz}, 2 \mathrm{H})$,
$3.46(\mathrm{t}, J=6.2 \mathrm{~Hz}, 1 \mathrm{H}), 1.87-1.68(\mathrm{~m}, 2 \mathrm{H}), 1.64-1.40(\mathrm{~m}, 4 \mathrm{H}), 1.23(\mathrm{~d}, J$ $=2.4 \mathrm{~Hz}, 12 \mathrm{H}), 0.07(\mathrm{~s}, 9 \mathrm{H}) ;{ }^{13} \mathbf{C}$ NMR $\left(75 \mathrm{MHz}, \mathrm{CD}_{2} \mathrm{Cl}_{2}\right) \delta 84.95,46.52,34.81,34.05,26.03,25.71$, 25.13, 0.90 (The carbon attached to boron was not observed due to quadrupolar relaxation); HRMS $\left(\mathrm{ESI}^{+}\right) \mathrm{m} / z$ calcd. for $\mathrm{C}_{14} \mathrm{H}_{30} \mathrm{BClNaO}_{3} \mathrm{Si}^{+}(\mathrm{M}+\mathrm{Na})^{+} 343.1638$, found 343.1637.

((5-Bromo-1-(4,4,5,5-tetramethyl-1,3,2-dioxaborolan-2-yl)pentyl)oxy)trimethylsilane (2k)

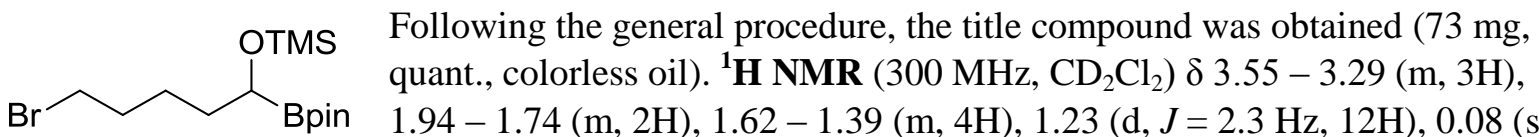
9H); ${ }^{13} \mathrm{C} \mathrm{NMR}\left(75 \mathrm{MHz}, \mathrm{CDCl}_{3}\right) \delta 84.87,35.34,34.59,34.16,26.34,25.94,25.62,0.80$ (The carbon attached to boron was not observed due to quadrupolar relaxation); HRMS HRMS (ESI $\left.{ }^{+}\right) \mathrm{m} / z$ calcd. for $\mathrm{C}_{14} \mathrm{H}_{30} \mathrm{BBrNaO}_{3} \mathrm{Si}^{+}(\mathrm{M}+\mathrm{Na})^{+}$387.1133, found 387.1127.

Trimethyl(3-(phenylthio)-1-(4,4,5,5-tetramethyl-1,3,2-dioxaborolan-2-yl)propoxy)silane (2l)

OTMS Following the general procedure, the title compound was obtained $(66 \mathrm{mg}$, quant., colorless oil). ${ }^{1} \mathbf{H} \mathbf{N M R}\left(400 \mathrm{MHz}, \mathrm{CD}_{2} \mathrm{Cl}_{2}\right) \delta 3.52-3.35(\mathrm{~m}, 1 \mathrm{H})$, Bpin $3.23(\mathrm{t}, J=7.0 \mathrm{~Hz}, 2 \mathrm{H}), 1.65-1.47(\mathrm{~m}, 4 \mathrm{H}), 1.47-1.30(\mathrm{~m}, 2 \mathrm{H}), 1.21(\mathrm{~d}, J$ $=3.4 \mathrm{~Hz}, 12 \mathrm{H}), 0.05(\mathrm{~s}, 9 \mathrm{H}) ;{ }^{13} \mathbf{C}$ NMR $\left(101 \mathrm{MHz}, \mathrm{CD}_{2} \mathrm{Cl}_{2}\right) \delta 84.98,52.81,35.10,30.24,26.04,25.71$, 24.96, 0.90 (The carbon attached to boron was not observed due to quadrupolar relaxation); HRMS $\left(\mathrm{ESI}^{+}\right) \mathrm{m} / z$ calcd. for $\mathrm{C}_{14} \mathrm{H}_{30} \mathrm{BN}_{3} \mathrm{NaO}_{3} \mathrm{Si}^{+}(\mathrm{M}+\mathrm{Na})^{+} 350.2042$, found 350.2040 .

\section{7-(4,4,5,5-Tetramethyl-1,3,2-dioxaborolan-2-yl)-7-((trimethylsilyl)oxy)heptyl 4-} methylbenzenesulfonate $(\mathbf{2 m})$

OTMS Following the general procedure, the title compound was obtained (97
mg, quant., colorless oil). ${ }^{1} \mathbf{H ~ N M R ~}\left(400 \mathrm{MHz}, \mathrm{CD}_{2} \mathrm{Cl}_{2}\right) \delta 7.76(\mathrm{~d}, J=$ $J=6.6 \mathrm{~Hz}, 1 \mathrm{H}), 2.45(\mathrm{~s}, 3 \mathrm{H}), 1.66-1.56(\mathrm{~m}, 2 \mathrm{H}), 1.52-1.41(\mathrm{~m}, 2 \mathrm{H}), 1.37-1.10(\mathrm{~m}, 18 \mathrm{H}), 0.06(\mathrm{~s}$, 9H); ${ }^{13} \mathbf{C}$ NMR $\left(101 \mathrm{MHz}, \mathrm{CD}_{2} \mathrm{Cl}_{2}\right) \delta 146.20,134.50,131.15,129.10,84.86,72.17,35.43,30.27$, $30.06,27.45,26.59,26.01,25.70,22.68,0.90$ (The carbon attached to boron was not observed due to quadrupolar relaxation); HRMS $\left(\mathrm{ESI}^{+}\right) \mathrm{m} / z$ calcd. for $\mathrm{C}_{23} \mathrm{H}_{41} \mathrm{BNaO}_{6} \mathrm{SSi}^{+}(\mathrm{M}+\mathrm{Na})^{+}$507.2378, found 507.2384. 

dione (2n)<smiles>COC(CCCCN1C(=O)c2ccccc2C1=O)Cc1ccccc1</smiles>

Following the general procedure, the title compound was obtained (86 mg, quant., colorless oil). ${ }^{1} \mathbf{H}$ NMR $\left(400 \mathrm{MHz}, \mathrm{CD}_{2} \mathrm{Cl}_{2}\right) \delta 7.84-$ $7.78(\mathrm{~m}, 2 \mathrm{H}), 7.74-7.67(\mathrm{~m}, 2 \mathrm{H}), 3.65(\mathrm{t}, J=7.2 \mathrm{~Hz}, 2 \mathrm{H}), 3.44(\mathrm{t}, J$ $=6.7 \mathrm{~Hz}, 1 \mathrm{H}), 1.72-1.60(\mathrm{~m}, 2 \mathrm{H}), 1.56(\mathrm{dt}, J=14.1,4.2 \mathrm{~Hz}, 2 \mathrm{H})$, $1.48-1.29(\mathrm{~m}, 2 \mathrm{H}), 1.20(\mathrm{~d}, J=4.8 \mathrm{~Hz}, 12 \mathrm{H}), 0.05(\mathrm{~s}, 9 \mathrm{H}) ;{ }^{13} \mathbf{C}$

NMR (101 MHz, $\left.\mathrm{CD}_{2} \mathrm{Cl}_{2}\right) \delta 169.58,135.13,133.61,124.24,84.91,39.23,35.17,29.88,26.01,25.71$, 25.09, 0.90 (The carbon attached to boron was not observed due to quadrupolar relaxation); HRMS $\left(\mathrm{ESI}^{+}\right) \mathrm{m} / z$ calcd. for $\mathrm{C}_{22} \mathrm{H}_{34} \mathrm{BNNaO}_{5} \mathrm{Si}^{+}(\mathrm{M}+\mathrm{Na})^{+}$454.2192, found 454.2192.

$N, 4-D i m e t h y l-N$-(7-(4,4,5,5-tetramethyl-1,3,2-dioxaborolan-2-yl)-7((trimethylsilyl)oxy)heptyl)benzenesulfonamide (2o)

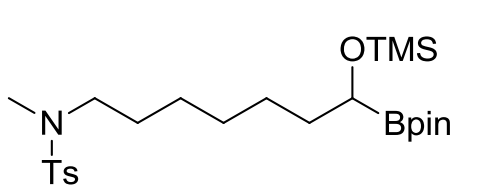

Following the general procedure, the title compound was obtained (100 mg, quant., colorless oil). ${ }^{1} \mathbf{H}$ NMR $\left(500 \mathrm{MHz}, \mathrm{CD}_{2} \mathrm{Cl}_{2}\right) \delta 7.66-$ $7.60(\mathrm{~m}, 2 \mathrm{H}), 7.36-7.31(\mathrm{~m}, 2 \mathrm{H}), 3.45(\mathrm{t}, J=6.7 \mathrm{~Hz}, 1 \mathrm{H}), 2.93(\mathrm{t}, J=$ $7.4 \mathrm{~Hz}, 2 \mathrm{H}), 2.67(\mathrm{~s}, 3 \mathrm{H}), 2.42(\mathrm{~s}, 3 \mathrm{H}), 1.54-1.46(\mathrm{~m}, 4 \mathrm{H}), 1.40-$ $1.27(\mathrm{~m}, 6 \mathrm{H}), 1.23(\mathrm{~d}, J=4.2 \mathrm{~Hz}, 12 \mathrm{H}), 0.07(\mathrm{~s}, 9 \mathrm{H}) ;{ }^{13} \mathbf{C} \mathbf{N M R}\left(126 \mathrm{MHz}, \mathrm{CD}_{2} \mathrm{Cl}_{2}\right) \delta 143.28,134.61$, 129.54, 127.30, 83.50, 50.12, 34.41, 34.19, 29.23, 27.52, 26.49, 26.22, 24.66, 24.37, 21.18, - 0.43 (The carbon attached to boron was not observed due to quadrupolar relaxation); HRMS (ESI $\left.{ }^{+}\right) \mathrm{m} / z$ calcd. for $\mathrm{C}_{24} \mathrm{H}_{44} \mathrm{BNNaO}_{5} \mathrm{SSi}^{+}(\mathrm{M}+\mathrm{Na})^{+}$520.2695, found 520.2699.

tert-Butylmethyl(7-(4,4,5,5-tetramethyl-1,3,2-dioxaborolan-2-yl)-7((trimethylsilyl)oxy)heptyl)carbamate (2p)<smiles>CN(CCCCCCC(O[Na])c1ccccc1)C(=O)OCc1ccccc1</smiles>

Following the general procedure, the title compound was obtained (100 mg, quant., colorless oil). ${ }^{\mathbf{1}} \mathbf{H}$ NMR (500 MHz, $\left.\mathrm{CD}_{2} \mathrm{Cl}_{2}\right) \delta 3.45(\mathrm{t}$, $J=6.7 \mathrm{~Hz}, 1 \mathrm{H}), 3.16(\mathrm{t}, J=7.3 \mathrm{~Hz}, 2 \mathrm{H}), 2.79(\mathrm{~s}, 3 \mathrm{H}), 1.56-1.44(\mathrm{~m}$, $4 \mathrm{H}), 1.42(\mathrm{~s}, 9 \mathrm{H}), 1.39-1.25(\mathrm{~m}, 6 \mathrm{H}), 1.23(\mathrm{~d}, J=4.3 \mathrm{~Hz}, 12 \mathrm{H}), 0.07$ $(\mathrm{s}, 9 \mathrm{H}) ;{ }^{13} \mathbf{C} \mathbf{N M R}\left(126 \mathrm{MHz}, \mathrm{CD}_{2} \mathrm{Cl}_{2}\right) \delta 155.49,83.47,78.54,48.75,34.24,33.74,29.42,28.14,26.65$, $26.33,24.65,24.35,-0.45$ (The carbon attached to boron was not observed due to quadrupolar relaxation); HRMS $\left(\mathrm{ESI}^{+}\right) \mathrm{m} / z$ calcd. for $\mathrm{C}_{22} \mathrm{H}_{46} \mathrm{BNNaO}_{5} \mathrm{Si}^{+}(\mathrm{M}+\mathrm{Na})^{+} 466.3131$, found 466.3135 .

Trimethyl(3-methyl-3-(phenylsulfonyl)-1-(4,4,5,5-tetramethyl-1,3,2-dioxaborolan-2yl)butoxy)silane (2q)

$\begin{array}{ll}\mathrm{PhO}_{2} \mathrm{~S} & \begin{array}{l}\text { Following the general procedure, the title compound was obtained }(85 \mathrm{mg}, \\ \text { quant., colorless oil). }{ }^{1} \mathbf{H} \text { NMR }\left(300 \mathrm{MHz}, \mathrm{CD}_{2} \mathrm{Cl}_{2}\right) \delta 7.85(\mathrm{t}, J=8.1 \mathrm{~Hz}, 2 \mathrm{H}) \text {, }\end{array} \\ 7.67(\mathrm{dd}, J=9.6,5.0 \mathrm{~Hz}, 1 \mathrm{H}), 7.60-7.49(\mathrm{~m}, 2 \mathrm{H}), 3.71(\mathrm{dd}, J=9.1,4.2 \mathrm{~Hz},\end{array}$ $1 \mathrm{H}), 2.05-1.78(\mathrm{~m}, 2 \mathrm{H}), 1.34(\mathrm{~d}, J=2.7 \mathrm{~Hz}, 6 \mathrm{H}), 1.22(\mathrm{~d}, J=0.7 \mathrm{~Hz}, 12 \mathrm{H}), 0.07(\mathrm{~s}, 9 \mathrm{H}) ;{ }^{13} \mathbf{C ~ N M R}$ $\left(75 \mathrm{MHz}, \mathrm{CD}_{2} \mathrm{Cl}_{2}\right) \delta 134.28,132.41,129.47,127.56,83.35,62.24,37.45,23.43,23.34,20.64,20.07$, 0.40 (The carbon attached to boron was not observed due to quadrupolar relaxation); HRMS (ESI $\left.{ }^{+}\right)$ $m / z$ calcd. for $\mathrm{C}_{20} \mathrm{H}_{35} \mathrm{BNaO}_{5} \mathrm{SSi}^{+}(\mathrm{M}+\mathrm{Na})^{+} 449.1960$, found 449.1968 .

\section{(4-Methoxy-1-(4,4,5,5-tetramethyl-1,3,2-dioxaborolan-2-yl)butoxy)trimethylsilane (2r)}

$\begin{array}{ll}\text { OTMS } & \begin{array}{l}\text { Following the general procedure, the title compound was obtained }(60 \mathrm{mg}, \\ \text { quant., colorless oil). }{ }^{1} \mathbf{H} \text { NMR }\left(400 \mathrm{MHz}, \mathrm{CD}_{2} \mathrm{Cl}_{2}\right) \delta 3.46(\mathrm{t}, J=6.3 \mathrm{~Hz}, 1 \mathrm{H}),\end{array}\end{array}$ 12H), 0.07 (s, 9H); ${ }^{13} \mathbf{C}$ NMR (101 MHz, $\left.\mathrm{CD}_{2} \mathrm{Cl}_{2}\right) \delta 84.94,74.07,59.45,32.15,27.93,26.02,25.73$, 0.90 (The carbon attached to boron was not observed due to quadrupolar relaxation); HRMS (ESI ${ }^{+}$) $m / z$ calcd. for $\mathrm{C}_{14} \mathrm{H}_{32} \mathrm{BO}_{4} \mathrm{Si}^{+}(\mathrm{M}+\mathrm{H})^{+}$303.2157, found 303.2167. 
OTMS Following the general procedure, the title compound was obtained Bpin
$(\mathrm{t}, J=6.5 \mathrm{~Hz}, 2 \mathrm{mg}), 3.45(\mathrm{t}, J=6.7 \mathrm{~Hz}, 1 \mathrm{H}), 1.57-1.44(\mathrm{~m}, 4 \mathrm{H})$, $1.40-1.27(\mathrm{~m}, 6 \mathrm{H}), 1.23(\mathrm{~d}, J=3.4 \mathrm{~Hz}, 12 \mathrm{H}), 0.89(\mathrm{~s}, 9 \mathrm{H}), 0.07(\mathrm{~s}, 9 \mathrm{H}), 0.04(\mathrm{~s}, 6 \mathrm{H}) ;{ }^{13} \mathbf{C}$ NMR $(101$ $\left.\mathrm{MHz}, \mathrm{CD}_{2} \mathrm{Cl}_{2}\right) \delta 84.81,64.46,35.60,34.19,30.78,27.67,27.08,27.03,26.00,25.70,19.50,0.90$, 4.29 (The carbon attached to boron was not observed due to quadrupolar relaxation); HRMS (ESI ${ }^{+}$) $m / z$ calcd. for $\mathrm{C}_{22} \mathrm{H}_{50} \mathrm{BO}_{4} \mathrm{Si}^{+}(\mathrm{M}+\mathrm{H})^{+} 445.3335$, found 445.3343.

\section{Trimethyl(3-(phenylthio)-1-(4,4,5,5-tetramethyl-1,3,2-dioxaborolan-2-yl)propoxy)silane (2t)}

OTMS Following the general procedure, the title compound was obtained ( $73 \mathrm{mg}$, quant.,

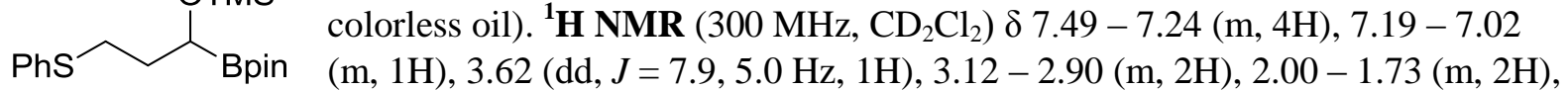
$1.23(\mathrm{~d}, J=3.5 \mathrm{~Hz}, 12 \mathrm{H}), 0.10(\mathrm{~s}, 9 \mathrm{H}) ;{ }^{13} \mathbf{C}$ NMR $\left(101 \mathrm{MHz}, \mathrm{CD}_{2} \mathrm{Cl}_{2}\right) \delta 138.39,130.13,129.54$, $126.69,85.17,35.13,31.09,26.05,25.72,0.90$ (The carbon attached to boron was not observed due to quadrupolar relaxation); HRMS $\left(\mathrm{ESI}^{+}\right) \mathrm{m} / z$ calcd. for $\mathrm{C}_{18} \mathrm{H}_{31} \mathrm{BNaO}_{3} \mathrm{SSi}^{+}(\mathrm{M}+\mathrm{Na})^{+} 389.1748$, found 389.1756.

\section{7-(4,4,5,5-Tetramethyl-1,3,2-dioxaborolan-2-yl)-7-((trimethylsilyl)oxy)heptyl benzoate (2u)}

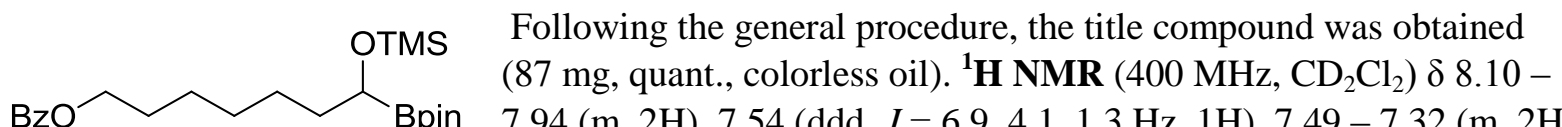
$4.27(\mathrm{t}, J=6.6 \mathrm{~Hz}, 2 \mathrm{H}), 3.45(\mathrm{t}, J=6.6 \mathrm{~Hz}, 1 \mathrm{H}), 1.81-1.66(\mathrm{~m}, 2 \mathrm{H}), 1.57-1.48(\mathrm{~m}, 2 \mathrm{H}), 1.47-1.31$ $(\mathrm{m}, 6 \mathrm{H}), 1.21(\mathrm{~d}, J=3.5 \mathrm{~Hz}, 12 \mathrm{H}), 0.06(\mathrm{~s}, 9 \mathrm{H}) ;{ }^{13} \mathbf{C} \mathbf{N M R}\left(101 \mathrm{MHz}, \mathrm{CD}_{2} \mathrm{Cl}_{2}\right) \delta 167.69,134.00$, $132.01,130.68,129.62,84.83,66.35,35.52,30.61,29.99,27.56,27.31,26.00,25.69,0.90$ (The carbon attached to boron was not observed due to quadrupolar relaxation); HRMS (ESI $\left.{ }^{+}\right) \mathrm{m} / \mathrm{z}$ calcd. for $\mathrm{C}_{23} \mathrm{H}_{40} \mathrm{BO}_{5} \mathrm{Si}^{+}(\mathrm{M}+\mathrm{H})^{+} 435.2733$, found 435.2729.

\section{7-(4,4,5,5-Tetramethyl-1,3,2-dioxaborolan-2-yl)-7-((trimethylsilyl)oxy)heptyl pivalate (2v)}

Following the general procedure, the title compound was obtained
$\left(82 \mathrm{mg}\right.$, quant., colorless oil). ${ }^{1} \mathbf{H}$ NMR $\left(300 \mathrm{MHz}, \mathrm{CD}_{2} \mathrm{Cl}_{2}\right) \delta 4.01(\mathrm{t}$, $1.44(\mathrm{~m}, 2 \mathrm{H}), 1.40-1.28(\mathrm{~m}, 6 \mathrm{H}), 1.23(\mathrm{~d}, J=2.3 \mathrm{~Hz}, 12 \mathrm{H}), 1.17(\mathrm{~s}, 9 \mathrm{H}), 0.07(\mathrm{~s}, 9 \mathrm{H}) ;{ }^{13} \mathbf{C ~ N M R}(101$ $\left.\mathrm{MHz}, \mathrm{CD}_{2} \mathrm{Cl}_{2}\right) \delta 179.52,84.82,65.59,39.87,35.50,30.54,29.89,28.27,27.51,27.16,26.00,25.70$, 0.90 (The carbon attached to boron was not observed due to quadrupolar relaxation); HRMS (ESI $\left.{ }^{+}\right)$ $m / z$ calcd. for $\mathrm{C}_{21} \mathrm{H}_{47} \mathrm{BNO}_{5} \mathrm{Si}^{+}\left(\mathrm{M}+\mathrm{NH}_{4}\right)^{+} 432.3311$, found 432.3315 .

7-(4,4,5,5-Tetramethyl-1,3,2-dioxaborolan-2-yl)-7-((trimethylsilyl)oxy)heptyl furan-2carboxylate (2w)<smiles>COC(CCCCCCOC(=O)c1ccco1)Cc1ccccc1</smiles>

Following the general procedure, the title compound was obtained (84 mg, quant., colorless oil). ${ }^{1} \mathbf{H}$ NMR $(400 \mathrm{MHz}$, $\left.\mathrm{CD}_{2} \mathrm{Cl}_{2}\right) \delta 7.77(\mathrm{dd}, J=3.7,1.3 \mathrm{~Hz}, 1 \mathrm{H}), 7.57(\mathrm{dd}, J=5.0,1.2$ $\mathrm{Hz}, 1 \mathrm{H}), 7.11(\mathrm{dd}, J=5.0,3.7 \mathrm{~Hz}, 1 \mathrm{H}), 4.26(\mathrm{t}, J=6.7 \mathrm{~Hz}$, $2 \mathrm{H}), 3.46(\mathrm{t}, J=6.6 \mathrm{~Hz}, 1 \mathrm{H}), 1.78-1.68(\mathrm{~m}, 2 \mathrm{H}), 1.59-1.50(\mathrm{~m}, 2 \mathrm{H}), 1.48-1.33(\mathrm{~m}, 6 \mathrm{H}), 1.23(\mathrm{~d}, J$ $=3.5 \mathrm{~Hz}, 12 \mathrm{H}), 0.07(\mathrm{~s}, 9 \mathrm{H}) ;{ }^{13} \mathbf{C} \mathbf{N M R}\left(101 \mathrm{MHz}, \mathrm{CD}_{2} \mathrm{Cl}_{2}\right) \delta 163.40,135.60,134.32,133.43,129.03$, 84.84, 66.54, 35.51, 30.57, 29.96, 27.54, 27.21, 26.00, 25.70, 0.90 (The carbon attached to boron was not observed due to quadrupolar relaxation); $\mathbf{H R M S}\left(\mathrm{ESI}^{+}\right) \mathrm{m} / z$ calcd. for $\mathrm{C}_{21} \mathrm{H}_{37} \mathrm{BNaO}_{6} \mathrm{Si}^{+}(\mathrm{M}+\mathrm{Na})^{+}$ 447.2345, found 447.2349. 
7-(4,4,5,5-Tetramethyl-1,3,2-dioxaborolan-2-yl)-7-((trimethylsilyl)oxy)heptyl furan-2carboxylate $(2 x)$<smiles>COC(CCCCCCOC(=O)c1cccs1)Cc1ccccc1</smiles>

Following the general procedure, the title compound was obtained ( $88 \mathrm{mg}$, quant., colorless oil). ${ }^{1} \mathbf{H}$ NMR $(400 \mathrm{MHz}$, $\left.\mathrm{CD}_{2} \mathrm{Cl}_{2}\right) \delta 7.59(\mathrm{dd}, J=1.7,0.8 \mathrm{~Hz}, 1 \mathrm{H}), 7.15(\mathrm{dd}, J=3.5,0.8$ $\mathrm{Hz}, 1 \mathrm{H}), 6.52(\mathrm{dd}, J=3.5,1.7 \mathrm{~Hz}, 1 \mathrm{H}), 4.25(\mathrm{t}, J=6.7 \mathrm{~Hz}$, $2 \mathrm{H}), 3.45(\mathrm{t}, J=6.6 \mathrm{~Hz}, 1 \mathrm{H}), 1.78-1.68(\mathrm{~m}, 2 \mathrm{H}), 1.51(\mathrm{t}, J=$ $6.8 \mathrm{~Hz}, 2 \mathrm{H}), 1.47-1.32(\mathrm{~m}, 6 \mathrm{H}), 1.23(\mathrm{~d}, J=3.5 \mathrm{~Hz}, 12 \mathrm{H}), 0.07(\mathrm{~s}, 9 \mathrm{H}) ;{ }^{13} \mathbf{C}$ NMR $(101 \mathrm{MHz}$, $\left.\mathrm{CD}_{2} \mathrm{Cl}_{2}\right) \delta 159.92,147.54,146.38,118.74,113.02,84.85,66.26,35.51,30.57,29.96,27.55,27.18$, $26.00,25.70,0.90$ (The carbon attached to boron was not observed due to quadrupolar relaxation); HRMS $\left(\mathrm{ESI}^{+}\right) \mathrm{m} / z$ calcd. for $\mathrm{C}_{21} \mathrm{H}_{38} \mathrm{BO}_{6} \mathrm{Si}^{+}(\mathrm{M}+\mathrm{H})^{+} 441.2297$, found 441.2294

\section{2-(5-(4,4,5,5-Tetramethyl-1,3,2-dioxaborolan-2-yl)-5-} ((trimethylsilyl)oxy)pentyl)benzo[d]isothiazol-3(2H)-one 1,1-dioxide (2y)

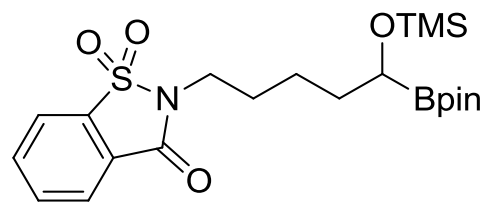

Following the general procedure but with toluene as solvent, the title compound was obtained (93 mg, quant., colorless oil). ${ }^{1} \mathbf{H}$ NMR $\left(400 \mathrm{MHz}, \mathrm{CD}_{2} \mathrm{Cl}_{2}\right) \delta 8.05-7.99(\mathrm{~m}, 1 \mathrm{H}), 7.94-7.89(\mathrm{~m}, 1 \mathrm{H}), 7.88$ $-7.80(\mathrm{~m}, 2 \mathrm{H}), 3.74(\mathrm{t}, J=7.6 \mathrm{~Hz}, 2 \mathrm{H}), 3.53-3.42(\mathrm{~m}, 1 \mathrm{H}), 1.83$ (tdd, $J=9.8,8.2,2.8 \mathrm{~Hz}, 2 \mathrm{H}), 1.65-1.54(\mathrm{~m}, 2 \mathrm{H}), 1.54-1.35(\mathrm{~m}$, $2 \mathrm{H}), 1.22(\mathrm{~d}, J=4.0 \mathrm{~Hz}, 12 \mathrm{H}), 0.07(\mathrm{~s}, 9 \mathrm{H}) ;{ }^{13} \mathbf{C ~ N M R}\left(101 \mathrm{MHz}, \mathrm{CD}_{2} \mathrm{Cl}_{2}\right) \delta 160.19,139.06,136.09$, $135.67,128.85,126.27,122.10,84.96,40.64,35.05,29.82,26.02,25.72,25.08,0.90$ (The carbon attached to boron was not observed due to quadrupolar relaxation); HRMS (ESI $\left.{ }^{+}\right) \mathrm{m} / z$ calcd. for $\mathrm{C}_{21} \mathrm{H}_{35} \mathrm{BNO}_{6} \mathrm{SSi}^{+}(\mathrm{M}+\mathrm{H})^{+}$468.2042, found 468.2048.

Triethyl(phenyl(4,4,5,5-tetramethyl-1,3,2-dioxaborolan-2-yl)methoxy)silane (2z)<smiles>S=S(=S)(Br)c1ccccc1</smiles>

Following the general procedure, the title compound was obtained (93 mg, quant., colorless oil). ${ }^{1} \mathbf{H}$ NMR $\left(300 \mathrm{MHz}, \mathrm{CD}_{2} \mathrm{Cl}_{2}\right) \delta 7.35(\mathrm{dt}, J=15.1,7.4 \mathrm{~Hz}, 4 \mathrm{H}), 7.22(\mathrm{t}$, $J=6.9 \mathrm{~Hz}, 1 \mathrm{H}), 4.68(\mathrm{~s}, 1 \mathrm{H}), 1.23(\mathrm{~d}, J=5.2 \mathrm{~Hz}, 12 \mathrm{H}), 0.98(\mathrm{t}, J=7.9 \mathrm{~Hz}, 9 \mathrm{H})$, $0.64(\mathrm{dt}, J=8.6,5.2 \mathrm{~Hz}, 6 \mathrm{H}) ;{ }^{13} \mathbf{C}$ NMR $\left(75 \mathrm{MHz}, \mathrm{CD}_{2} \mathrm{Cl}_{2}\right) \delta 142.92,128.01$, $126.04,125.52,83.86,24.41,24.19,6.60,4.56$ (The carbon attached to boron was not observed due to quadrupolar relaxation); $\mathbf{H R M S}\left(\mathrm{ESI}^{+}\right) \mathrm{m} / z$ calcd. for $\mathrm{C}_{19} \mathrm{H}_{33} \mathrm{BNaO}_{3} \mathrm{Si}^{+}(\mathrm{M}+\mathrm{Na})^{+}$ 371.2184, found 371.2210.

Triethyl(phenyl(4,4,5,5-tetramethyl-1,3,2-dioxaborolan-2-yl)methoxy)silane (2aa)<smiles>COC(Cc1ccccc1)c1ccc(C)cc1</smiles>

Following the general procedure, the title compound was obtained $(93 \mathrm{mg}$, quant., colorless oil). ${ }^{1} \mathbf{H}$ NMR $\left(400 \mathrm{MHz}, \mathrm{CD}_{2} \mathrm{Cl}_{2}\right) \delta 7.21(\mathrm{~d}, J=8.0 \mathrm{~Hz}, 2 \mathrm{H}), 7.12(\mathrm{~d}, J$ $=7.9 \mathrm{~Hz}, 2 \mathrm{H}), 4.57(\mathrm{~s}, 1 \mathrm{H}), 2.32(\mathrm{~s}, 3 \mathrm{H}), 1.20(\mathrm{~d}, J=7.6 \mathrm{~Hz}, 12 \mathrm{H}), 0.09(\mathrm{~s}, 9 \mathrm{H})$; ${ }^{13}$ C NMR $\left(101 \mathrm{MHz}, \mathrm{CD}_{2} \mathrm{Cl}_{2}\right) \delta 140.75,137.18,130.10,127.36,85.14,25.69$, $25.59,22.06,0.90$ (The carbon attached to boron was not observed due to quadrupolar relaxation); $\mathbf{H R M S}\left(\mathrm{ESI}^{+}\right) \mathrm{m} / z$ calcd. for $\mathrm{C}_{17} \mathrm{H}_{29} \mathrm{BNaO}_{3} \mathrm{Si}^{+}(\mathrm{M}+\mathrm{Na})^{+} 343.1871$, found 343.1878 .

Triethyl((4-methoxyphenyl)(4,4,5,5-tetramethyl-1,3,2-dioxaborolan-2-yl)methoxy)silane (2ab)<smiles>COc1ccc(C(O[Na-])c2ccccc2)cc1</smiles>

Following the general procedure, the title compound was obtained $(93 \mathrm{mg}$, quant., colorless oil). ${ }^{1} \mathbf{H}$ NMR $\left(300 \mathrm{MHz}, \mathrm{CD}_{2} \mathrm{Cl}_{2}\right) \delta 7.29(\mathrm{~d}, J=8.7 \mathrm{~Hz}, 2 \mathrm{H})$, $6.88(\mathrm{~d}, J=8.7 \mathrm{~Hz}, 2 \mathrm{H}), 4.60(\mathrm{~s}, 1 \mathrm{H}), 3.82(\mathrm{~s}, 3 \mathrm{H}), 1.23(\mathrm{~d}, J=6.1 \mathrm{~Hz}, 12 \mathrm{H})$, $0.97(\mathrm{t}, J=7.9 \mathrm{~Hz}, 9 \mathrm{H}), 0.63(\mathrm{dt}, J=8.5,5.2 \mathrm{~Hz}, 6 \mathrm{H}) ;{ }^{13} \mathbf{C ~ N M R}(75 \mathrm{MHz}$, $\left.\mathrm{CD}_{2} \mathrm{Cl}_{2}\right) \delta 158.25,134.80,127.07,113.44,83.77,55.06,24.40,24.24,6.60$, 4.58 (The carbon attached to boron was not observed due to quadrupolar relaxation); HRMS (ESI $\left.{ }^{+}\right)$ $\mathrm{m} / \mathrm{z}$ calcd. for $\mathrm{C}_{20} \mathrm{H}_{35} \mathrm{BNaO}_{4} \mathrm{Si}^{+}(\mathrm{M}+\mathrm{Na})^{+} 401.2290$, found 401.2300 . 
<smiles>COc1ccccc1C(OC)c1ccccc1</smiles>

Following the general procedure, the title compound was obtained (93 mg, quant., colorless oil). ${ }^{1} \mathbf{H}$ NMR $\left(400 \mathrm{MHz}, \mathrm{CD}_{2} \mathrm{Cl}_{2}\right) \delta 7.37(\mathrm{ddd}, J=7.5,1.4,1.0 \mathrm{~Hz}, 1 \mathrm{H})$, $7.26-7.13(\mathrm{~m}, 1 \mathrm{H}), 6.96(\mathrm{td}, J=7.5,0.8 \mathrm{~Hz}, 1 \mathrm{H}), 6.84(\mathrm{~d}, J=8.1 \mathrm{~Hz}, 1 \mathrm{H}), 4.66(\mathrm{~s}$, $1 \mathrm{H}), 3.80(\mathrm{~s}, 3 \mathrm{H}), 1.24(\mathrm{~d}, J=2.5 \mathrm{~Hz}, 12 \mathrm{H}), 0.13(\mathrm{~s}, 9 \mathrm{H}) ;{ }^{13} \mathbf{C ~ N M R}(101 \mathrm{MHz}$, $\left.\mathrm{CD}_{2} \mathrm{Cl}_{2}\right) \delta 156.95,132.98,128.39,127.92,121.77,110.68,84.83,56.21,25.76$, 25.62, 0.90 (The carbon attached to boron was not observed due to quadrupolar relaxation); HRMS $\left(\mathrm{ESI}^{+}\right) \mathrm{m} / z$ calcd. for $\mathrm{C}_{17} \mathrm{H}_{29} \mathrm{BNaO}_{4} \mathrm{Si}^{+}(\mathrm{M}+\mathrm{Na})^{+} 359.1820$, found 359.1829 .

Using electron-rich Lewis base-borane $\mathrm{NHC}-\mathrm{BH}_{3}$ as $\mathrm{B}-\mathrm{H}$ donor:<smiles>CC(=O)CCc1ccccc1</smiles>

1a, $0.2 \mathrm{mmol}$<smiles>[B]c1n(C)cc[n+]1C</smiles>

$0.24 \mathrm{mmol}$<smiles>CC(C)(C)CCCCCOC(C)(C)C</smiles>
Toluene, $\mathrm{rt}, 12 \mathrm{~h}$<smiles>CN[B]C([B]OC(C)CCc1ccccc1)O[Sn]</smiles>

$2 \mathbf{a}^{\prime}, 61 \%$ yield

To an oven-dried screw-cap $5 \mathrm{~mL}$ reaction tube was charged the $\mathrm{NHC}^{-\mathrm{BH}_{3}}(0.24 \mathrm{mmol}, 1.2$ equiv.). The tube was then evacuated and back-filled with argon for 3 times. Subsequently, toluene ( $2 \mathrm{~mL})$ was added followed by acylsilane substrate (1a, 0.2 mmol, 1.0 equiv.) under argon. Once added, the tube was closed and the reaction mixture was stirred and irradiated in a photobox with blue LEDs ( $\lambda \max =$ $420 \mathrm{~nm}$ ) for $12 \mathrm{~h}$. The mixture was concentrated and purified by column chromatography to give the product 2a'(39 mg, 61\% yield, white solid). ${ }^{1} \mathbf{H}$ NMR (400 MHz, $\left.\mathrm{CD}_{2} \mathrm{Cl}_{2}\right) \delta 7.27-7.18(\mathrm{~m}, 4 \mathrm{H}), 7.15$ $-7.09(\mathrm{~m}, 1 \mathrm{H}), 6.84(\mathrm{~s}, 2 \mathrm{H}), 3.77(\mathrm{~s}, 6 \mathrm{H}), 3.54-3.41(\mathrm{~m}, 1 \mathrm{H}), 2.79-2.56(\mathrm{~m}, 2 \mathrm{H}), 1.86-1.66(\mathrm{~m}$, 2H), -0.15 (s, 9H).; ${ }^{13}$ C NMR (101 MHz, $\left.\mathrm{CD}_{2} \mathrm{Cl}_{2}\right)$ 145.73, 129.48, 129.01, 126.02, 121.26, 43.81, $37.24,35.29,0.90$. (The carbon attached to boron was not observed due to quadrupolar relaxation); HRMS $\left(\mathrm{ESI}^{+}\right) \mathrm{m} / z$ calcd. for $\mathrm{C}_{17} \mathrm{H}_{29} \mathrm{BN}_{2} \mathrm{NaOSi}^{+}(\mathrm{M}+\mathrm{Na})^{+} 339.2034$, found 339.2056.

\section{Other attempts:}

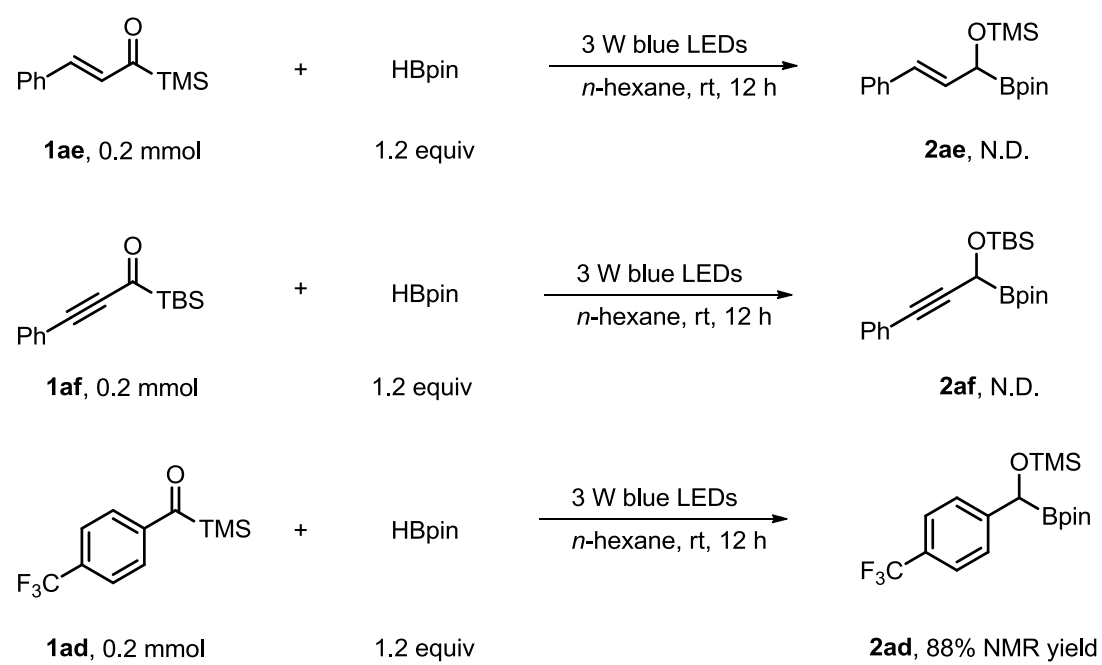

Unfortunately no desired products were generated when the R of substrate $\mathbf{1}$ was alkenyl (1ae) or alkynyl (1af) group. We also tried the substrate 1ad with an electron-deficient aryl, providing the desired product in $88 \%$ NMR yield. However, 2ad decomposed quickly when purification by column chromatography. 


\subsection{Solvent Screening}

An oven-dried screw-cap $5 \mathrm{~mL}$ reaction tube was evacuated and back-filled with argon for 3 times. Subsequently, solvent $(2 \mathrm{~mL})$ was added followed by acylsilane substrate $\mathbf{1 a}(0.2 \mathrm{mmol}, 1.0$ equiv.) and HBpin (0.24 mmol, 1.2 equiv.) under argon. Once added, the tube was closed and the reaction mixture was stirred and irradiated in a photobox with blue LEDs $(\lambda \max =420 \mathrm{~nm})$ for $12 \mathrm{~h}$.

\begin{tabular}{|c|c|c|}
\hline$\overbrace{1 \mathrm{TMS}}^{\mathrm{O}, 0.2 \mathrm{mmol}}$ & $\begin{array}{l}3 \mathrm{~W} \text { blue LEDs } \\
\text { solvent, rt, } 12 \mathrm{~h}\end{array}$ & $\left.\right|_{\mathbf{2 a}} ^{\text {OTMS }}$ \\
\hline Entry & Base & $3 \mathbf{a}(\%)^{a}$ \\
\hline 1 & $\mathrm{DMF}$ & 30 \\
\hline 2 & $\mathrm{CH}_{3} \mathrm{CN}$ & 75 \\
\hline 3 & THF & 80 \\
\hline 4 & $n$-Hexane & quant. \\
\hline 5 & $\mathrm{CH}_{2} \mathrm{Cl}_{2}$ & quant. \\
\hline 6 & Toluene & quant. \\
\hline 7 & $n$-Hexane $(1 \mathrm{~mL})$ & quant. \\
\hline 8 & $n$-Hexane (3 mL) & quant. \\
\hline 9 & $n$-Hexane, darkness & N.D. \\
\hline 10 & $n$-Hexane, darkness, $100^{\circ} \mathrm{C}$ & N.D. \\
\hline
\end{tabular}

${ }^{a}$ Determined by crude ${ }^{1} \mathrm{H}-\mathrm{NMR}$ with $\mathrm{CH}_{2} \mathrm{Br}_{2}$ as internal standard. Quantitative yields were obtained by concentration of the reaction mixture and evaporation of volatiles.

\subsection{Gram-scale Reaction}

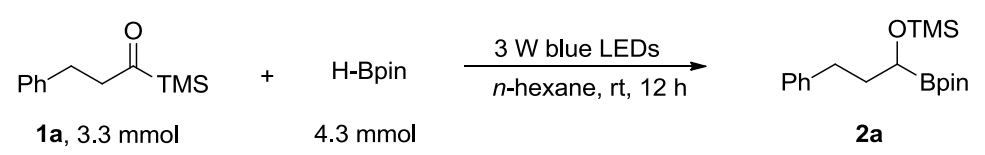

To an oven-dried $100 \mathrm{~mL}$ reaction flask was evacuated and back-filled with argon for 3 times. Subsequently, $n$-hexane $(30 \mathrm{~mL})$ was added followed by $\mathbf{1 a}(3.3 \mathrm{mmol}, 1.0$ equiv.) and HBpin (4.3 mmol, 1.3 equiv.) under argon. Once added, the reaction mixture was stirred and irradiated in a photobox with blue LEDs $(\lambda \max =420 \mathrm{~nm})$ for $20 \mathrm{~h}$. The mixture was concentrated to directly afford the product (1.100 g, quantitative yield) as colorless oil without column chromatography (excess of HBpin was removed in vacuo). 


\subsection{Sensitivity Assessment}
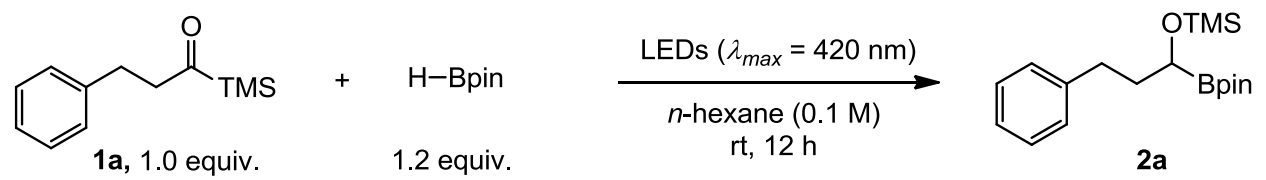

To an oven-dried $10 \mathrm{~mL}$ Schlenk tube equipped with a stir bar was added starting material 1a $(20.5 \mathrm{mg}$, $0.1 \mathrm{mmol}, 1.0$ equiv.), HBPin ( $15.5 \mathrm{mg}, 0.12 \mathrm{mmol}, 1.2$ equiv.) and $n$-hexane (1.0 mL, $0.1 \mathrm{M})$. The reaction mixture was stirred and irradiated with $5 \mathrm{~W}$ blue LEDs $\left(\lambda_{\max }=420 \mathrm{~nm}\right)$ for $12 \mathrm{~h}$. The resulting mixture was analyzed by GC-FID with mesitylene as the internal standard.

Standard conditions: $n=0.1 \mathrm{mmol}, c=0.1 \mathrm{M}, V=1 \mathrm{~mL}$, inert atmosphere, $T=25^{\circ} \mathrm{C}, I=0.07 \mathrm{~W} / \mathrm{cm}^{2}$, $d=2.2 \mathrm{~cm}$.

Stock solution: $n(\mathbf{1 a})=1.2 \mathrm{mmol}, n(\mathrm{HBPin})=1.44 \mathrm{mmol}, c=0.111 \mathrm{M}, V=10.8 \mathrm{~mL}: 0.246 \mathrm{~g} \mathbf{1 a}$, $0.186 \mathrm{~g}$ HBPin, $n$-hexane: $10.8 \mathrm{~mL}$.

Stock solution 'big scale': $n(\mathbf{1 a})=2.1 \mathrm{mmol}, n($ HBPin $)=2.52 \mathrm{mmol}, V=21 \mathrm{~mL}: 0.430 \mathrm{~g} \mathrm{1a}, 0.326 \mathrm{~g}$ HBPin, $n$-hexane: $21 \mathrm{~mL}$.

\begin{tabular}{l|l|l|c|c} 
Number & Experiment & Preparation & Yield (\%) & Deviation (\%) \\
\hline 1 & High $c$ & $0.9 \mathrm{~mL}$ stock sol. & quant. & 0 \\
2 & Low $c$ & $0.9 \mathrm{~mL}$ stock sol. $+0.2 \mathrm{~mL} n$-hexane & quant. & 0 \\
3 & High $\mathrm{H}_{2} \mathrm{O}$ & Std. cond. $+10 \mu \mathrm{L} \mathrm{H}_{2} \mathrm{O}$ & 6 & 94 \\
5 & Low $\mathrm{O}_{2}$ & Std. cond. + degassed & quant. & 0 \\
6 & High $\mathrm{O}_{2}$ & Std. cond. $+10 \mathrm{~mL}$ air & 11 & 89 \\
7 & Low $T$ & Std. cond., $T=15^{\circ} \mathrm{C}$ & 98 & 2 \\
8 & High $T$ & Std. cond., $T=33^{\circ} \mathrm{C}$ & quant. & 0 \\
9 & Low $I$ & Std. cond., $I=0.006 \mathrm{~W} / \mathrm{cm}^{2}, d=8.8 \mathrm{~cm}$ & 83 & 17 \\
10 & Control $I$ & Std. cond., $I=0.550 \mathrm{~W} / \mathrm{cm}^{2}, d=0.5 \mathrm{~cm}$ & quant. & 0 \\
11 & Control 2 & Std. cond., water jacketed flask & quant. & 0 \\
12 & Big scale & $20 \mathrm{~mL}$ stock sol. Big scale & quant. & 0
\end{tabular}




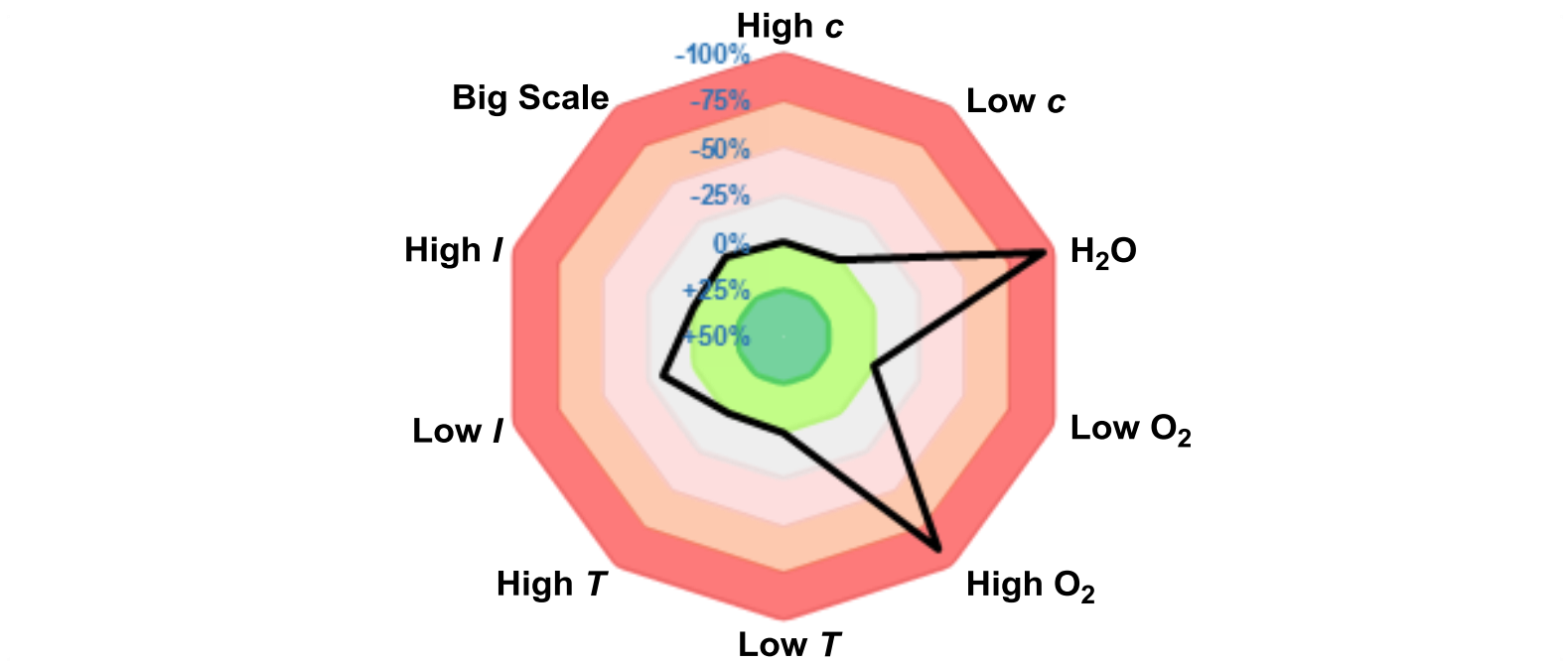

\section{Characterization Studies with UV/Vis Spectroscopy}

In order to investigate possible adduct formation between 1a and HBpin, UV/vis absorption studies were performed. All absorption spectra were recorded on a JASCO V-730 two-beam photospectrometer using the following parameter set: data Interval $0.5 \mathrm{~nm}$, bandwidth $1.0 \mathrm{~nm}$, response time $0.06 \mathrm{~s}$, scan speed $400 \mathrm{~nm} \mathrm{~min}^{-1}$, baseline correction.

Initially, individual absorption spectra of all reaction components ( $c=0.01 \mathrm{M}$ in $n$-Hexane, Figure S3) and absorption spectra of all binary mixtures $(c=0.01 \mathrm{M}$ in $n$-Hexane, Figure S4).

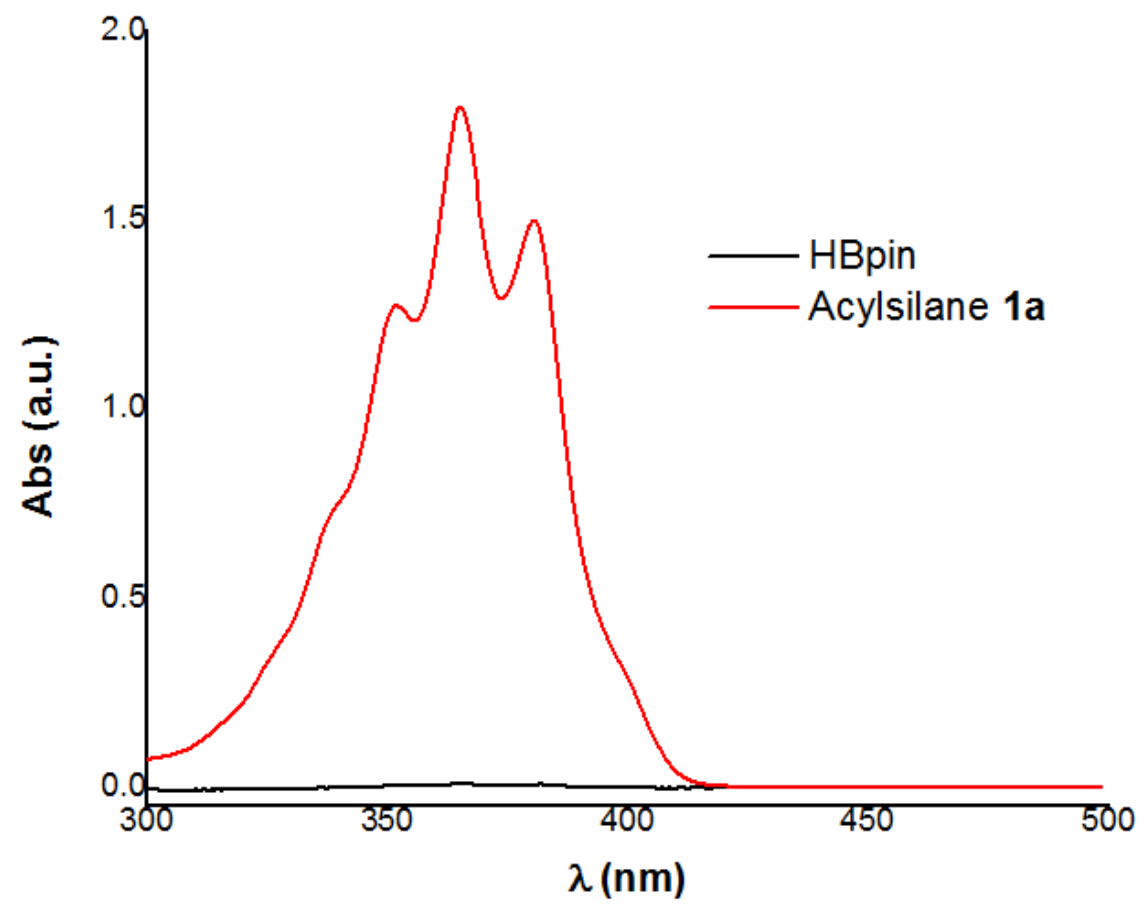

Figure S3: Individual absorption Spectra of acylsilane 1a, HBpin (0.01 M in $n$-Hexane). 


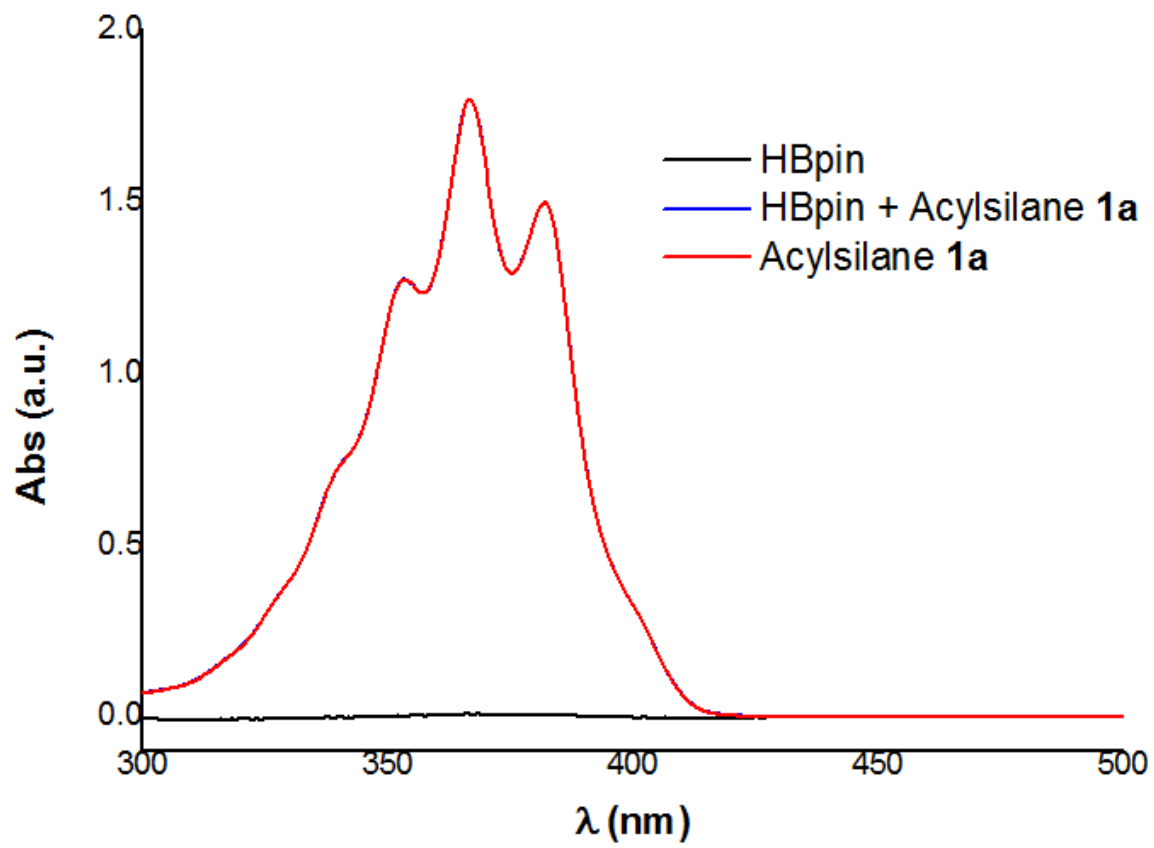

Figure S4: Absorption Spectra of the mixture of 1a and HBpin in 1:1 ratio ( $c=0.01 \mathrm{M}$ in $n$-Hexane).

\section{Control Experiments}

An oven-dried screw-cap $5 \mathrm{~mL}$ reaction tube was evacuated and back-filled with argon for 3 times. Subsequently, $n$-Hexane $(2 \mathrm{~mL})$ was added followed by $\mathbf{1 a}(0.2 \mathrm{mmol}, 1.0$ equiv. $)$ and HBpin $(0.24$ mmol, 1.2 equiv.) under argon. Once added, the tube was closed and the reaction mixture was stirred and irradiated in a photobox with $5 \mathrm{~W}$ blue LEDs $(\lambda \max =455 \mathrm{~nm})$ for $12 \mathrm{~h}$. The mixture was analyzed by ${ }^{1} \mathrm{H}-\mathrm{NMR}$ with $\mathrm{CH}_{2} \mathrm{Br}_{2}$ as internal standard, indicating the yield of $\mathbf{2 a}$ was less than $5 \%$ yield.

(1)

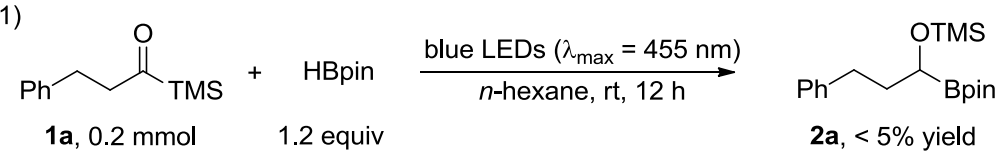

To an oven-dried screw-cap $5 \mathrm{~mL}$ reaction tube was charged the $\left[\operatorname{Ir}\left(\mathrm{dF}\left(\mathrm{CF}_{3}\right) \text { ppy }\right)_{2}(\mathrm{dtbbpy})\right] \mathrm{PF}_{6}(\mathrm{Ir}-\mathbf{F}$, 0.004 mmol, 0.02 equiv.). The tube was then evacuated and back-filled with argon for 3 times. Subsequently, $\mathrm{CH}_{2} \mathrm{Cl}_{2}(2 \mathrm{~mL})$ was added followed by $\mathbf{1 a}(0.2 \mathrm{mmol}, 1.0$ equiv.) and HBpin $(0.24$ mmol, 1.2 equiv.) under argon. Once added, the tube was closed and the reaction mixture was stirred and irradiated in a photobox with $5 \mathrm{~W}$ blue LEDs $(\lambda \max =455 \mathrm{~nm})$ for $12 \mathrm{~h}$. The mixture was analyzed by ${ }^{1} \mathrm{H}-\mathrm{NMR}$ with $\mathrm{CH}_{2} \mathrm{Br}_{2}$ as internal standard, indicating quantitative yield of $\mathbf{2 a}$ was obtained.

(2)

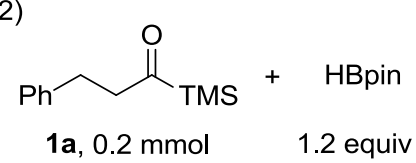

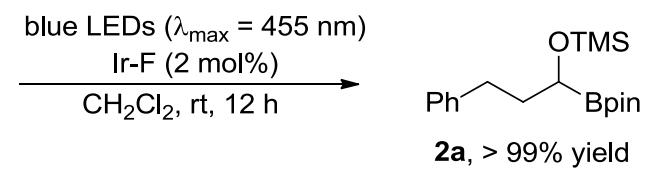

To an oven-dried screw-cap $5 \mathrm{~mL}$ reaction tube was charged the trans-stilbene ( $0.2 \mathrm{mmol}, 1.0$ equiv.). The tube was then evacuated and back-filled with argon for 3 times. Subsequently, $n$-Hexane $(2 \mathrm{~mL})$ was added followed by $1 \mathbf{a}(0.2 \mathrm{mmol}, 1.0$ equiv.) and HBpin ( $0.24 \mathrm{mmol}, 1.2$ equiv.) under argon. Once added, the tube was closed and the reaction mixture was stirred and irradiated in a photobox with $3 \mathrm{~W}$ blue LEDs $(\lambda \max =420 \mathrm{~nm})$ for $12 \mathrm{~h}$. The mixture analyzed by ${ }^{1} \mathrm{H}-\mathrm{NMR}$ with $\mathrm{CH}_{2} \mathrm{Br}_{2}$ as internal 
standard. As shown below, only $10 \%$ yield of $2 \mathbf{a}$ was obtained while trans-stilbene was converted in $83 \%$ to cis-stilbene.

(3)

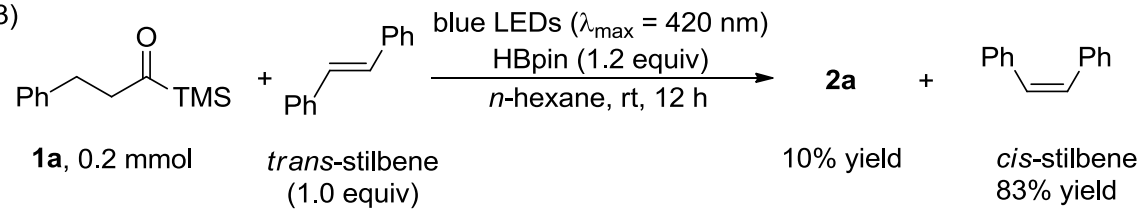

To an oven-dried screw-cap $5 \mathrm{~mL}$ reaction tube was charged the trans-stilbene ( $0.2 \mathrm{mmol}, 1.0$ equiv.). The tube was then evacuated and back-filled with argon for 3 times. Subsequently, $n$-Hexane $(2 \mathrm{~mL})$ was added followed by $\mathbf{1 a}(0.2 \mathrm{mmol}, 1.0$ equiv.) under argon. Once added, the tube was closed and the reaction mixture was stirred and irradiated in a photobox with $3 \mathrm{~W}$ blue LEDs $(\lambda \max =420 \mathrm{~nm})$ for $12 \mathrm{~h}$. The mixture analyzed by ${ }^{1} \mathrm{H}-\mathrm{NMR}$ with $\mathrm{CH}_{2} \mathrm{Br}_{2}$ as internal standard, indicating $95 \%$ of $\mathbf{1 a}$ recovered while trans-stilbene was converted in $85 \%$ to cis-stilbene.

(4)

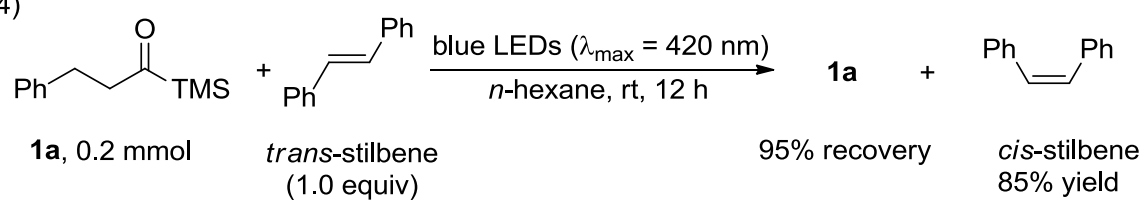

Kinetic isotope effect experiment: An oven-dried screw-cap $5 \mathrm{~mL}$ reaction tube was evacuated and back-filled with argon for 3 times. Subsequently, $n$-hexane $(2 \mathrm{~mL})$ was added followed by acylsilane substrate 1a ( $0.2 \mathrm{mmol}, 1.0$ equiv.), mixture of HBpin and DBPin in 1:1 ratio (0.24 mmol, 1.2 equiv.) under argon. Once added, the tube was closed and the reaction mixture was stirred and irradiated in a photobox with blue LEDs $(\lambda \max =420 \mathrm{~nm})$ for $0.5 \mathrm{~h}$. The mixture was concentrated and analyzed by NMR. The reaction showed only a minor $K_{H} / K_{D}(1.8: 1)$, which is in good agreement with the proposed rate-determining step since cleavage of $\mathrm{H}-\mathrm{B} / \mathrm{D}-\mathrm{B}$ bonds is not involved in RDS (According to the reaction profile shown in Figure S5, TS 3-4 has the highest energy on the reaction path from photoexcited substrate $3 \mathbf{T}^{*}$ to product 2a. Consequently, this rearrangement should be the ratedetermining step after photoexcitation.).

(5)
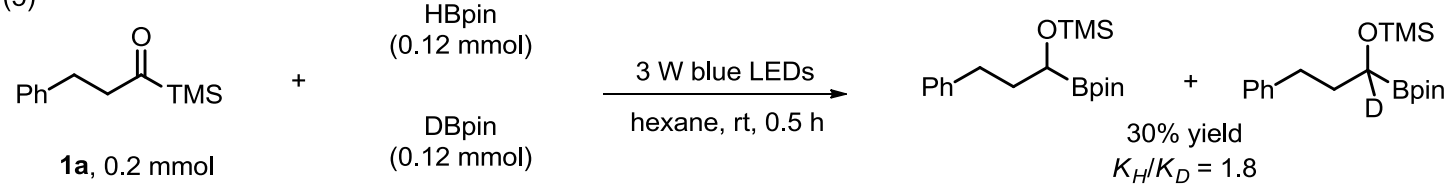

\section{Computational Analysis}

All computational studies were carried out using the ORCA 4.1.1 software package. ${ }^{[2,3]}$ Structures were visualized with Jmol. ${ }^{[4]}$

\subsection{Reaction Profile}

The geometries of all relevant stationary points were optimized using the B3LYP hybrid functional ${ }^{[5]}$ and Ahlrichs' def2-SVP double-zeta basis set ${ }^{6}$. All structures were confirmed to be local minima on the potential energy surface by the absence of negative eigenvalues of the Hessian after harmonic frequency analysis at the same level. No symmetrical or internal coordinate constraints were applied. Higher energy conformers were taken into account, but not included in the final calculation. Additional single point computations on the previously optimized geometries were performed using the def2-TZVPP triple-zeta basis set ${ }^{[7]}$. The reported free energy values (in $\mathrm{kcal} / \mathrm{mol}$ ) were obtained as 
the sum of the single point electronic energies and the respective free energy corrections (ZPVE, thermal corrections, enthalpy, entropy), as obtained from the harmonic frequency calculation.

Acylsilane 1a and its exited states were analyzed via time-dependent density functional theory (TDDFT) calculations (first ten exited states). With this, an absorption band at $357.8 \mathrm{~nm}$, which corresponds to an excitation from the HOMO to the LUMO, could be computed. This matches the experimental spectrum, which shows a similar maximum and tails into the visible light region. Further absorption bands where detected at $245.5 \mathrm{~nm}, 240.2 \mathrm{~nm}$ and $234.9 \mathrm{~nm}$. To estimate the free energy value of the singlet state $3 S^{*}$, a TDDFT geometry optimization and frequency calculation was conducted (using the ORCA Iroot keyword for state 2).

Following geometric relaxation to excited singlet state $3 \mathrm{~S}^{*}$, ISC to the triplet state $3 \mathbf{T}^{*}$ is computed to be favorable by $\Delta \mathrm{G}_{\mathrm{ISC}}=-17.1 \mathrm{kcal} / \mathrm{mol}$. The acylsilane from its triplet state $3 \mathrm{~T}^{*}$, can then undergo a 1,2-silyl shift, which shows an activation barrier of $11.4 \mathrm{kcal} / \mathrm{mol}$ and leads to triplet siloxycarbene 4T. This step is also exergonic by $\Delta \mathrm{G}_{3 \mathrm{~T}-4 \mathrm{~T}}=-9.2 \mathrm{kcal} / \mathrm{mol}$. Following, the triplet intermediate $4 \mathrm{~T}$ can undergo ISC to form the singlet carbene $\mathbf{4 S}$, which shows to be energetically favored (for a more detailed investigation of the carbene singlet/triplet gap see next chapter).

The singlet intermediate $\mathbf{4 S}$ could then interact with HBpin in three different pathways to insert into the B-H bond. Firstly, a nucleophilic attack to the boron atom could take place, subsequently after forming intermediate $\mathbf{X}$, a 1,2-hydride shift could lead to product $\mathbf{2 a}$. Since geometry optimization of this intermediate $\mathbf{X}$, starting from different singlet and triplet geometries, directly converged to the final product 2a, this pathway does not seem favorable (for a more detailed investigation, see next chapter). Second, a nucleophilic abstraction of the hydrogen atom can be considered. Here, a barrier of more than $148.2 \mathrm{kcal} / \mathrm{mol}$ would have to be overcome to form intermediate $\mathbf{Y}$ and therefore does not seem reasonable. Eventually, a concerted addition of HBpin (TS 4s-2a) was considered. Here, an activation barrier of $20.5 \mathrm{kcal} / \mathrm{mol}$ was calculated, which appears to be the most viable pathway. 


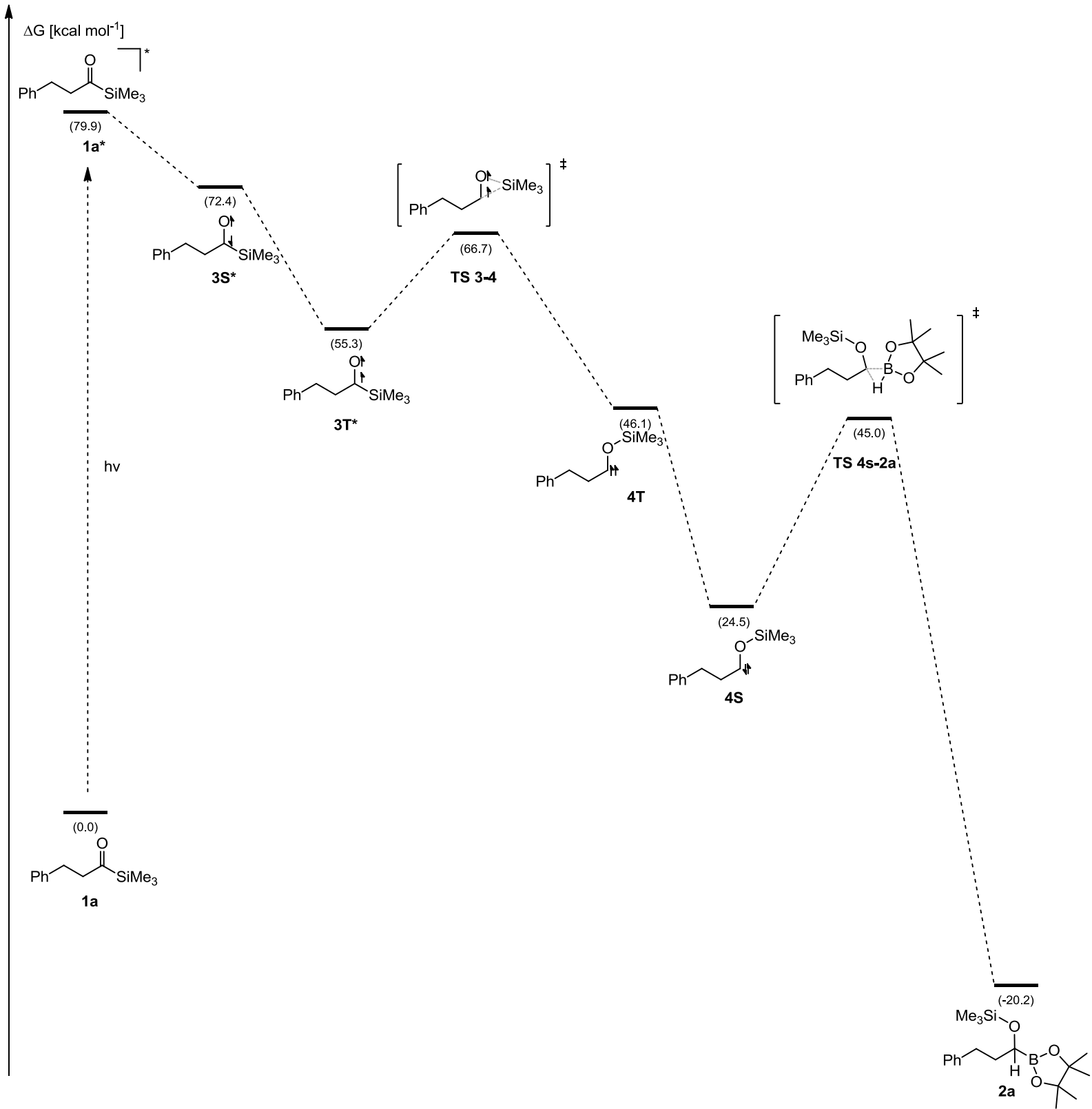

Figure S5: Reaction profile of the photo exitation of acylsilane, leading to a siloxycarbene and its reaction with HBpin. 


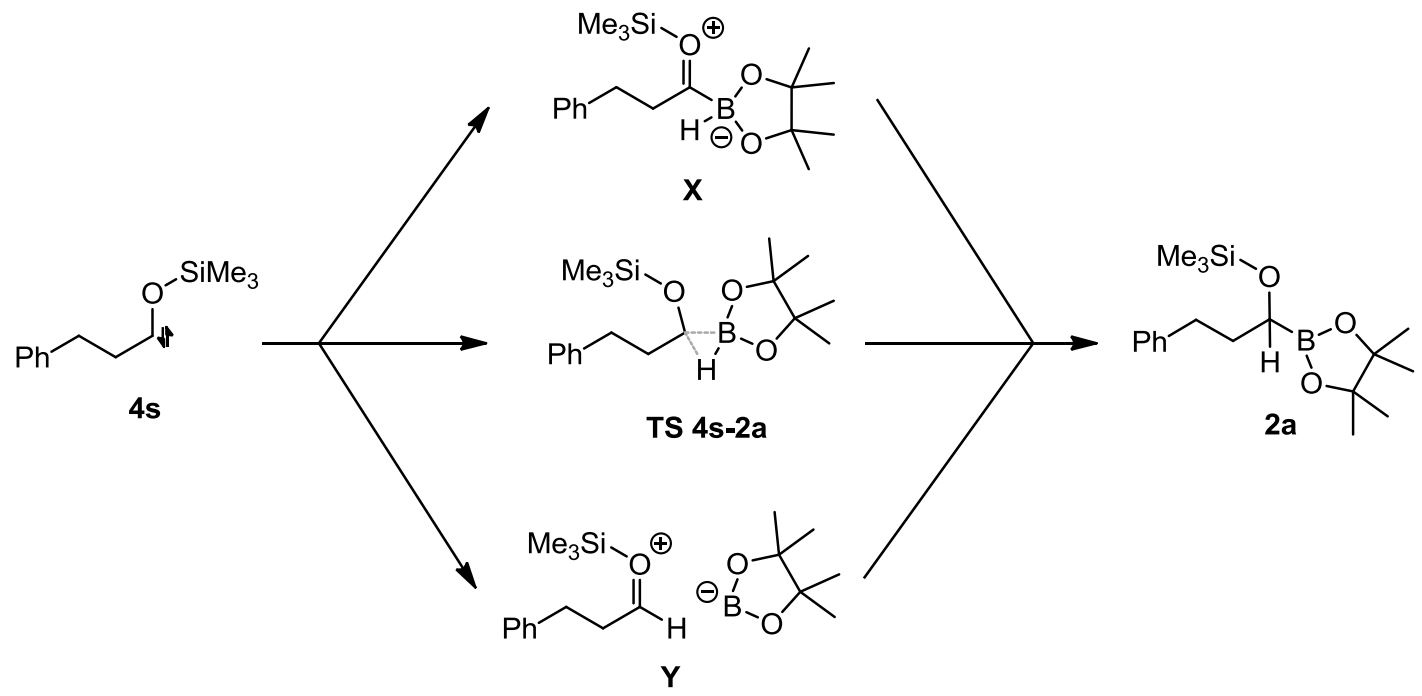

Figure S6: Reasonable reaction pathways for the addition of the siloxycarbene to HBpin.

Table S1: Stationary point energies of relevant structures. Energy values are given in $E_{h}$.

\begin{tabular}{|c|c|c|c|c|c|c|}
\hline & Free Enthalpy & SP Energy & ZPVE & $\begin{array}{c}\text { Thermal } \\
\text { Correction }\end{array}$ & $\begin{array}{c}\text { Enthalpy } \\
\text { Correction }\end{array}$ & $\begin{array}{c}\text { Entropy } \\
\text { Correction }\end{array}$ \\
\hline 1a & -832.47933014 & -832.70202053 & 0.26626104 & 0.01710942 & 0.00094421 & 0.06162428 \\
\hline 1a* & -832.35202097 & -832.57466143 & 0.26625461 & 0.01711354 & 0.00094421 & 0.06167190 \\
\hline 3S & -832.36399642 & -832.58668681 & 0.26626104 & 0.01710942 & 0.00094421 & 0.06162428 \\
\hline 3T & -832.39114047 & -832.61079314 & 0.26442900 & 0.01742038 & 0.000944421 & 0.06314092 \\
\hline TS 3-4 & -832.37458366 & -832.59423633 & 0.26442900 & 0.01742038 & 0.00094421 & 0.06314092 \\
\hline 4T & -832.40584015 & -832.62560440 & 0.26492237 & 0.01732324 & 0.00094421 & 0.06342557 \\
\hline 4S & -832.44020999 & -832.66117943 & 0.26467742 & 0.01728171 & 0.000944421 & 0.06193390 \\
\hline HBpin & -411.62209871 & -411.77855173 & 0.18935997 & 0.00951862 & 0.00094421 & 0.04336978 \\
\hline TS 4s-2a & -1244.02668800 & -1244.42666713 & 0.45486551 & 0.02796914 & 0.00094421 & 0.08379973 \\
\hline 2a & -1244.13064923 & -1244.53757835 & 0.46069731 & 0.02737567 & 0.00094421 & 0.08208807 \\
\hline Ya & -410.96733118 & -411.10965118 & 0.17526280 & 0.00958991 & 0.00094421 & 0.04347692 \\
\hline Yb & -832.85590246 & -833.09069667 & 0.27867372 & 0.01741711 & 0.00094421 & 0.06224083 \\
\hline
\end{tabular}

\subsection{Analysis of Siloxycarbene}

To further analyze whether a triplet or a singlet carbene inserts into $\mathrm{B}-\mathrm{H}$ bond further computational methods where applied. Here, the geometries of all relevant stationary points were optimized using the M062X hybrid functional ${ }^{[7]}$ and the def2-TZVPP triple-zeta basis set ${ }^{[6]}$. Additional single point computations on the previously optimized geometries were performed using a) the M062X functional ${ }^{[7]}$ with the def2-QZVPP quadruple-zeta basis set $^{[6]}$ b) the B2PLYP double hybrid functional ${ }^{[8]}$ with the def2-TZVPP triple-zeta basis set ${ }^{[6]}$. For all used methods, the singlet state $\mathbf{4 S}$ is energetically highly favored for the siloxycarbene $\left(\Delta \mathrm{G}_{\mathrm{ISC}}{ }^{\text {M062X/def2-QZVPP }}=-23.2 \mathrm{kcal} / \mathrm{mol}, \Delta \mathrm{G}_{\mathrm{ISC}}{ }^{\text {B2PLYP/def2-TZVPP }}=-27.4\right.$ $\mathrm{kcal} / \mathrm{mol})$.

Table S2: Stationary point energies of relevant structures. Energy values are given in Eh.

\begin{tabular}{|c|c|c|c|c|c|c|c|c|}
\hline & $\begin{array}{c}\text { Free Enthalpy } \\
\text { def2-QZVPP }\end{array}$ & $\begin{array}{c}\text { Free Enthalpy } \\
\text { B2PLYP }\end{array}$ & $\begin{array}{c}\text { Single point } \\
\text { def2-QZVPP }\end{array}$ & $\begin{array}{c}\text { Single point } \\
\text { B2PLYP }\end{array}$ & ZPVE & $\begin{array}{c}\text { Thermal } \\
\text { Correction }\end{array}$ & $\begin{array}{c}\text { Enthalpy } \\
\text { Correction }\end{array}$ & $\begin{array}{c}\text { Entropy } \\
\text { Correction }\end{array}$ \\
\hline 4S & -832.60580020 & -834.18491621 & -832.82969357 & -834.40880958 & 0.26764921 & 0.01722549 & 0.00094421 & 0.06192554 \\
\hline 4T & -832.56878028 & -834.14128184 & -832.79242936 & -834.36493092 & 0.26811545 & 0.01701449 & 0.00094421 & 0.06242507 \\
\hline
\end{tabular}

1a 


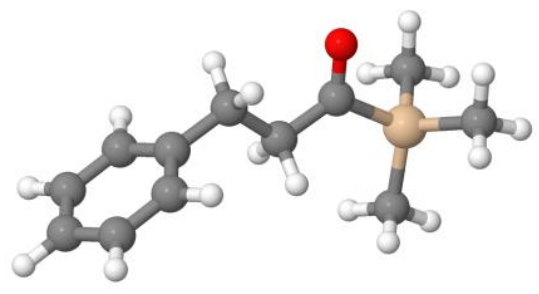

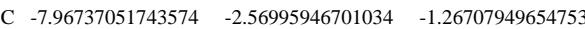

$\begin{array}{llll}\text { C } & -7.30963848251899 & -3.23758268738387 & -0.22914816297729\end{array}$

$\begin{array}{llll}\text { C } & -6.23084292501565 & -2.63268038127726 & 0.42258555610610\end{array}$

$\begin{array}{llll}\text { C } & -5.78610430042121 & -1.35213724491404 & 0.05313831822948\end{array}$

C $\quad-6.45925288380717 \quad-0.69305173647834 \quad-0.98924947108927$

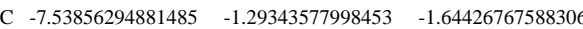

$\begin{array}{llll}\text { C } & -4.59454357462307 & -0.71604365186412 & 0.73396006926882\end{array}$

$\begin{array}{llll}\text { C } & -3.26167101726586 & -1.05402019789175 & 0.05123117583118\end{array}$

H $\quad-4.54081285348946 \quad-1.03520326864762 \quad 1.78711630373311$

H $\quad-4.70860899916467 \quad 0.37950726821336 \quad 0.75890216032011$

$\begin{array}{llll}\text { C } & -2.04052063384339 & -0.42714753061397 & 0.71051731943479\end{array}$

H $\quad-3.26782535065857 \quad-0.74330691287001 \quad-1.01165465138006$

$\begin{array}{llll}\text { H } & -3.09965807998571 & -2.14878209248371 & 0.01213296170472\end{array}$

$\begin{array}{llll}\text { O } & -2.15087163514780 & 0.27839017359465 & 1.69993830854840\end{array}$

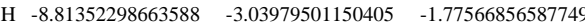

H $\quad-7.64151296573965 \quad-4.23314201043520 \quad 0.07881947797489$

H $\quad-5.72771326707259 \quad-3.16031109502790 \quad 1.23918501987123$

H $\quad-6.13637949153366 \quad 0.30978647414762 \quad-1.28665044008902$

H $\quad-8.05058289075728 \quad-0.75935505633541 \quad-2.44969621590285$

Si $-0.22476503697094 \quad-0.70131355561223 \quad 0.04283724590552$

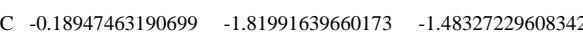

$\begin{array}{llll}\text { H } & -0.60013677202064 & -2.81998954609432 & -1.26528392399447\end{array}$

H $\quad 0.84557615600865 \quad-1.95869918962999 \quad-1.83985513525505$

H $\quad-0.77079592027388 \quad-1.39124412837950 \quad-2.31669930902495$

$\begin{array}{llll}\text { C } & 0.44113642281685 & 1.01947630650464 & -0.35776088507744\end{array}$

$\begin{array}{llll}\text { H } & 0.29271598460348 & 1.68852116896810 & 0.50526484039701\end{array}$

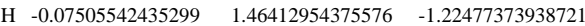

H $\quad 1.51945894258174 \quad 0.98674267247419 \quad-0.58770471322859$

C $0.73906594730514 \quad-1.46934433288332 \quad 1.47324074888343$

H $\quad 0.39935025189113 \quad-2.49614898222320 \quad 1.68804095369432$

H $\quad 0.59805255638430 \quad-0.86748812109711 \quad 2.38557152730097$

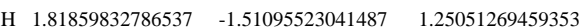

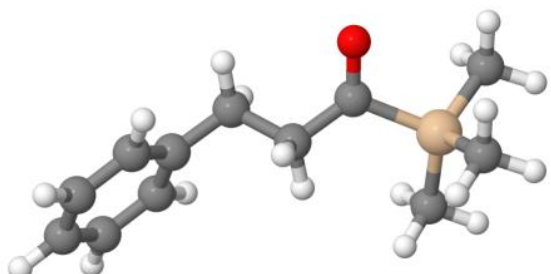

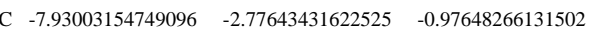
$\begin{array}{llll}\text { C } & -7.55199304351236 & -2.75037811941268 & 0.36987772352759\end{array}$
$\begin{array}{llll}\text { C } & -6.47122578116707 & -1.96502282708445 & 0.78043214692637\end{array}$
C $\quad-5.74770908847232 \quad-1.18981385616799 \quad-0.14228456756654$
$\begin{array}{llll}\text { C } & -6.13951559741459 & -1.22684225174811 & -1.49121516069989\end{array}$
$\begin{array}{llll}\text { C } & -7.22003414714142 & -2.01041551501593 & -1.90650792568537\end{array}$
$\begin{array}{llll}\text { C } & -4.55212940575318 & -0.37707776253327 & 0.29803300623386\end{array}$
$\begin{array}{llll}\text { C } & -3.24692832688083 & -1.21108021835273 & 0.30398266787283\end{array}$
$\begin{array}{llll}\text { H } & -4.71381464923778 & 0.02204599057484 & 1.31255134148914\end{array}$
$\begin{array}{llll}\text { H } & -4.41361739946829 & 0.49044661385840 & -0.36714896070229\end{array}$
$\begin{array}{llll}\text { C } & -2.03897621941938 & -0.39797482313993 & 0.75934715870569\end{array}$
$\begin{array}{llll}\text { H } & -3.05541461617720 & -1.61112939999629 & -0.70231767264648\end{array}$
H $\quad-3.38512141516683 \quad-2.08208525777281 \quad 0.97648631830775$
$\begin{array}{llll}\text { O } & -2.02395524324059 & 0.12756586365492 & 1.93008371064202\end{array}$

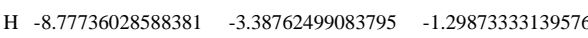
$\begin{array}{llll}\text { H } & -8.10484025705096 & -3.34130863081977 & 1.10547576887108\end{array}$
$\begin{array}{llll}\text { H } & -6.18723119888862 & -1.94487726127903 & 1.83726216268674\end{array}$
H $\quad-5.59323460622507 \quad-0.62573074541040 \quad-2.2249336840744$

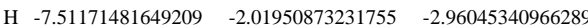
Si $-0.26985773960275 \quad-0.67382296338590 \quad 0.05231107915506$
C $\quad-0.46071212597893 \quad-1.19125384513437 \quad-1.75526024137399$
H $\quad-0.93490319177158 \quad-2.18034958512374 \quad-1.85983025260526$

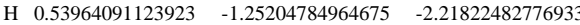
$\begin{array}{llll}\text { H } & -1.05253374345465 & -0.45936987035948 & -2.32795446485042\end{array}$
$\begin{array}{llll}\text { C } & 0.64791174746043 & 0.96621597308012 & 0.19967037840964\end{array}$
$\begin{array}{llll}\text { H } & 0.69045138745916 & 1.29231682416590 & 1.25148454688331\end{array}$
$\begin{array}{llll}\text { H } & 0.14334989831447 & 1.75773961249893 & -0.37739696346752\end{array}$
H $\quad 1.68257681775219 \quad 0.87410130371840 \quad-0.17278655302760$
$\begin{array}{llll}\text { C } & 0.64242198884261 & -2.00838109190637 & 1.03634987528108\end{array}$
$\begin{array}{llll}\text { Н } & 0.14361457307619 & -2.98748293601595 & 0.94925405767367\end{array}$
H $0.68000729683379 \quad-1.74191699168811 \quad 2.10522757115983$
$\begin{array}{llll}\text { H } & 1.68061082491324 & -2.12300234017668 & 0.67793116301709\end{array}$

TS 3-4 


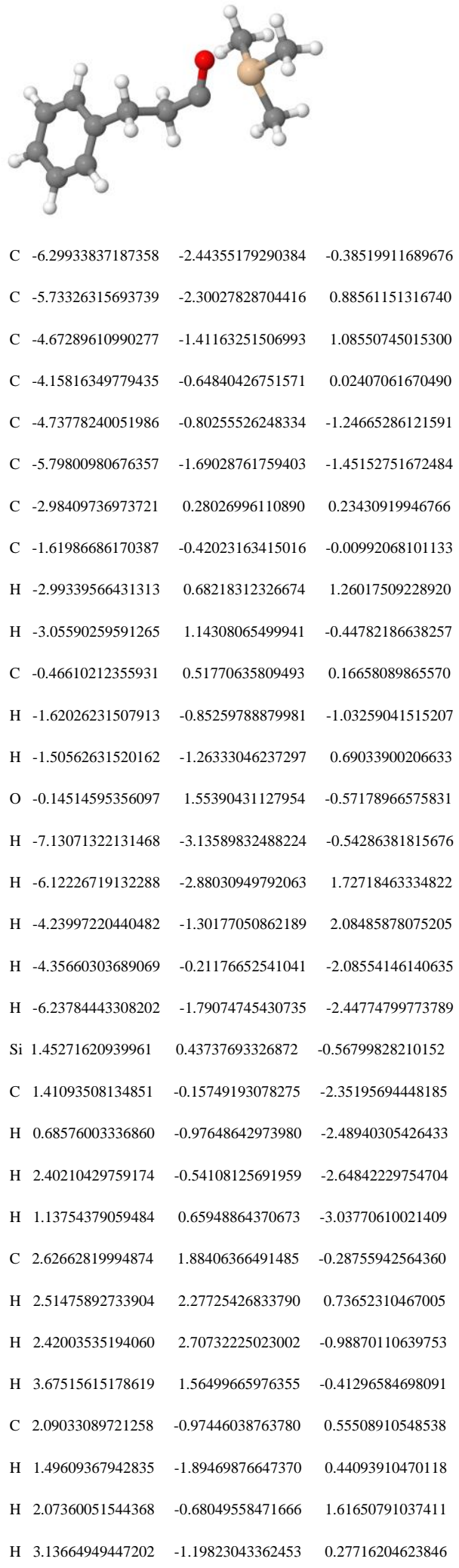

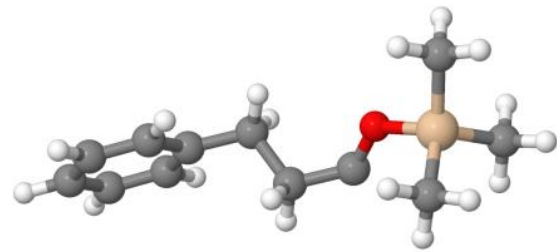

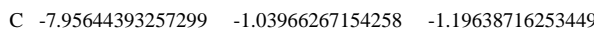

$\begin{array}{llll}\text { C } & -7.65018548308217 & -2.37879387464069 & -0.93413961793814\end{array}$

C $\quad-6.56032832820245 \quad-2.70369555323736 \quad-0.12120543434679$

$\begin{array}{llll}\text { C } & -5.75486942596809 & -1.70167441436937 & 0.44660833272615\end{array}$

$\begin{array}{llll}\text { C } & -6.07562405816167 & -0.36104517921275 & 0.17342734380648\end{array}$

$\begin{array}{llll}\text { C } & -7.16480718165317 & -0.03081607712657 & -0.63817078029087\end{array}$

$\begin{array}{llll}\text { C } & -4.54984443046720 & -2.05653372536693 & 1.28600355278973\end{array}$

$\begin{array}{llll}\text { C } & -3.25770664312705 & -2.24614974883338 & 0.43710535456720\end{array}$

$\begin{array}{llll}\text { H } & -4.73536367048839 & -2.99090348793651 & 1.84037923635249\end{array}$

H $\quad-4.36230099557572 \quad-1.27136408321766 \quad 2.03617804965290$

$\begin{array}{llll}\text { C } & -2.06897412044691 & -2.63694269000425 & 1.23718956729913\end{array}$

H $\quad-3.03053556566610 \quad-1.30822442580596 \quad-0.09620919731634$

H $\quad-3.46246115583113 \quad-3.00556674148082 \quad-0.34927723002608$

$\begin{array}{llll}\text { O } & -1.98133205286122 & -3.74937626589196 & 1.96706482382961\end{array}$

Si $-0.65286534356469 \quad-4.83381673328447 \quad 2.06152394438020$

$\begin{array}{llll}\text { C } & 0.79742171197136 & -3.91180933780412 & 2.82012497859610\end{array}$

H $\quad 0.53435652449574 \quad-3.51449721845535 \quad 3.81418401479919$

H $\quad 1.09773006274933 \quad-3.06218046842660 \quad 2.18510025611977$

H $1.67229470061758 \quad-4.57346128752519 \quad 2.93827938727894$

$\begin{array}{llll}\text { C } & -0.25130734581511 & -5.44547553706997 & 0.32962834698102\end{array}$

H $0.00297806869572 \quad-4.60206868328822 \quad-0.33311921159945$

H $\quad-1.10872389671057 \quad-5.97846337172718 \quad-0.11326894743538$

H $\quad 0.60808318528841 \quad-6.13715933297359 \quad 0.34239579573067$

C $-1.26844146571213 \quad-6.20957723564725 \quad 3.17635758020929$

$\begin{array}{llll}\text { H } & -0.48413692651044 & -6.96957123057987 & 3.33136181282597\end{array}$

H $\quad-2.14651360127822 \quad-6.71323470703924 \quad 2.74012977560567$

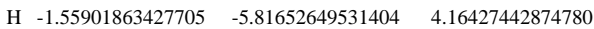

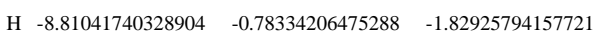

H $\quad-8.26612379483137 \quad-3.17525129985955 \quad-1.36135705174415$

$\begin{array}{llll}\text { H } & -6.33244242637582 & -3.75448039493248 & 0.08394546580492\end{array}$

$\begin{array}{llll}\text { H } & -5.46486130055027 & 0.43565714519022 & 0.60985622739392\end{array}$

H $\quad-7.39898407079914 \quad 1.01971419215655 \quad-0.83246370068820$

\section{T (B3LYP)}


4T (M062X)

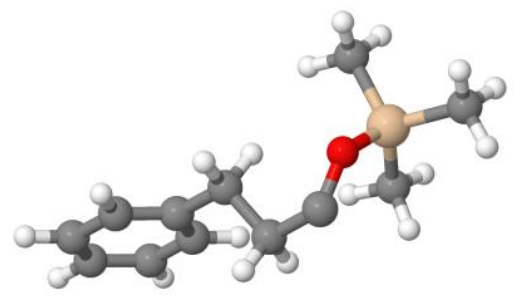

$\begin{array}{llll}\text { C } & -7.715432 & -0.642571 & 0.563201\end{array}$

$\begin{array}{llll}\text { C } & -6.715847 & -1.079234 & 1.424240\end{array}$

$\begin{array}{llll}\text { C } & -5.599978 & -1.730346 & 0.920539\end{array}$

$\begin{array}{llll}\text { C } & -5.456709 & -1.955088 & -0.448708\end{array}$

$\begin{array}{llll}\text { C } & -6.464494 & -1.511149 & -1.300340\end{array}$

$\begin{array}{llll}\text { C } & -7.587043 & -0.862102 & -0.800598\end{array}$

$\begin{array}{llll}\text { C } & -4.241859 & -2.665298 & -0.978407\end{array}$

$\begin{array}{llll}\text { C } & -2.949568 & -1.843755 & -0.819174\end{array}$

$\begin{array}{llll}\text { H } & -4.380732 & -2.918518 & -2.030223\end{array}$

$\begin{array}{llll}\text { H } & -4.117016 & -3.610617 & -0.444318\end{array}$

$\begin{array}{llll}\text { C } & -1.757814 & -2.599166 & -1.289654\end{array}$

$\begin{array}{llll}\mathrm{H} & -2.846630 & -1.554660 & 0.236275\end{array}$

$\begin{array}{llll}\text { H } & -3.034585 & -0.911539 & -1.380759\end{array}$

$\begin{array}{llll}\text { O } & -1.638880 & -3.918787 & -1.261510\end{array}$

$\begin{array}{llll}\text { Si } & -1.369849 & -4.951167 & 0.031607\end{array}$

$\begin{array}{llll}\text { C } & 0.329673 & -5.677568 & -0.178032\end{array}$

H $\quad 1.094485 \quad-4.900311 \quad-0.173773$

H $\quad 0.393468 \quad-6.207822 \quad-1.129780$

$\begin{array}{llll}\mathrm{H} & 0.556507 & -6.387335 & 0.619095\end{array}$

$\begin{array}{llll}\text { C } & -2.656581 & -6.295784 & -0.066705\end{array}$

$\begin{array}{llll}\text { H } & -2.633088 & -6.781761 & -1.043263\end{array}$

H $\quad-3.663903 \quad-5.909700 \quad 0.093531$

$\begin{array}{llll}\text { H } & -2.466171 & -7.058464 & 0.691236\end{array}$

$\begin{array}{llll}\text { C } & -1.539333 & -3.953930 & 1.597032\end{array}$

H $\quad-1.297567 \quad-4.553934 \quad 2.475498$

$\begin{array}{llll}\text { H } & -2.561844 & -3.587529 & 1.711732\end{array}$

$\begin{array}{llll}\mathrm{H} & -0.871828 & -3.090467 & 1.576787\end{array}$

$\begin{array}{llll}\text { H } & -8.589239 & -0.139615 & 0.954705\end{array}$

$\begin{array}{llll}\text { H } & -6.809992 & -0.917341 & 2.489776\end{array}$

$\begin{array}{llll}\text { H } & -4.830119 & -2.078773 & 1.599743\end{array}$

$\begin{array}{llll}\text { H } & -6.373610 & -1.685196 & -2.365583\end{array}$

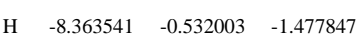

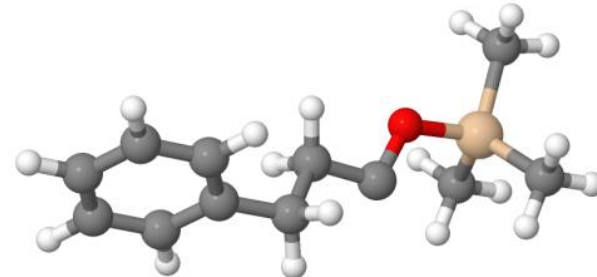

$\begin{array}{llll}\text { C } & -7.86852684186733 & -0.48223850098466 & 0.46303220322835\end{array}$

$\begin{array}{llll}\text { C } & -6.52196957399280 & -0.81486581170913 & 0.63709739122468\end{array}$

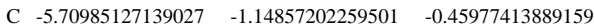

$\begin{array}{llll}\text { C } & -6.28890392520844 & -1.13685783108697 & -1.73936507946692\end{array}$

$\begin{array}{llll}\text { C } & -7.63520953617927 & -0.80519949490225 & -1.91928609089353\end{array}$

C $-4.26226988632366 \quad-1.54304616893346 \quad-0.26077735345856$

$\begin{array}{llll}\text { C } & -4.06696184838742 & -3.04730068916731 & 0.00234710438643\end{array}$

$\begin{array}{llll}\text { H } & -3.82726781771826 & -0.98611124012978 & 0.58495332669737\end{array}$

$\begin{array}{llll}\text { H } & -3.67637501261923 & -1.25978987764899 & -1.15144144849837\end{array}$

$\begin{array}{llll}\text { C } & -2.65939079406251 & -3.41297866159157 & 0.38132619675980\end{array}$

H $\quad-4.45642268224160 \quad-3.67432333851455 \quad-0.82450797627215$

$\begin{array}{llll}\text { H } & -4.64950874529691 & -3.33386147550770 & 0.90478831801437\end{array}$

$\begin{array}{llll}\text { O } & -2.41970418118832 & -4.64909103772298 & 0.01228473165164\end{array}$

Si $-0.84908085350558 \quad-5.31898430425623 \quad 0.41758905712678$

$\begin{array}{llll}\text { C } & -0.93345854434734 & -7.05346324580152 & -0.29341938216035\end{array}$

H $-1.10021500367516 \quad-7.02905645071964 \quad-1.38286457009079$

$\begin{array}{llll}\text { H } & -1.75545544088234 & -7.63000293661875 & 0.16178994503363\end{array}$

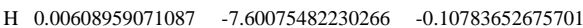

$\begin{array}{llll}\text { C } & -0.69890165727753 & -5.32493917724084 & 2.28570619260840\end{array}$

$\begin{array}{llll}\text { H } & -1.49562353069393 & -5.93305591208449 & 2.74515432423276\end{array}$

$\begin{array}{llll}\text { H } & -0.78365442521377 & -4.29787239579242 & 2.67399090182638\end{array}$

H $\quad 0.27178232985768 \quad-5.74134899017086 \quad 2.60388050346128$

$\begin{array}{llll}\text { C } & 0.45226446465629 & -4.26704736548439 & -0.42592495989269\end{array}$

H $\quad 1.46689132187634 \quad-4.63767982845602 \quad-0.20238968889523$

$\begin{array}{llll}\text { H } & 0.37515628951048 & -3.22447871305905 & -0.07984505911242\end{array}$

H $\quad 0.32094034023072 \quad-4.27658690069824 \quad-1.52057431531656$

H $\quad-9.48336799032541 \quad-0.21448486354295 \quad-0.95607721415878$

$\begin{array}{llll}\text { H } & -8.48031216913939 & -0.22088009985503 & 1.33115576826731\end{array}$

$\begin{array}{llll}\text { H } & -6.08946229867003 & -0.80968453414548 & 1.64275197191739\end{array}$

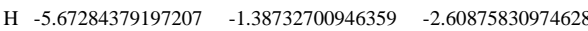

$\begin{array}{llll}\text { H } & -8.06364836054756 & -0.79937344187733 & -2.92557304436466\end{array}$

\section{S (M062X)}




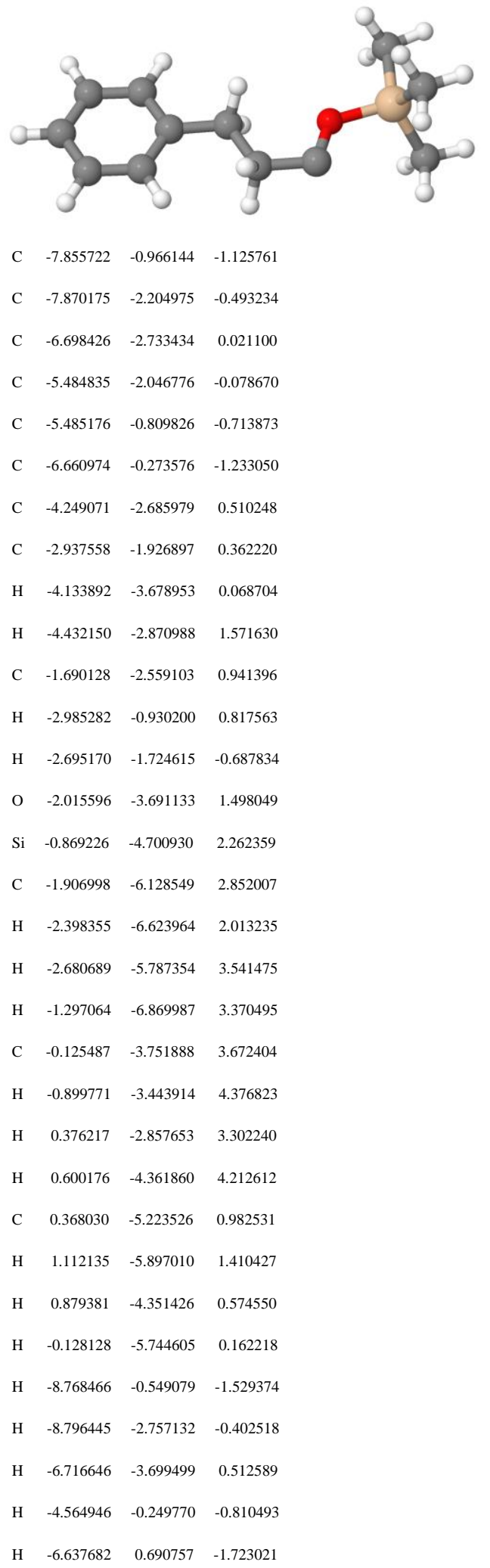

\section{HBpin}

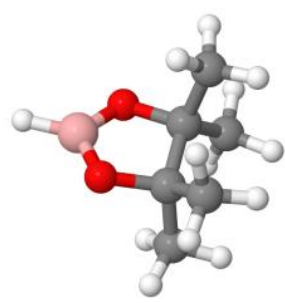

\begin{tabular}{cllll} 
B & -3.69755214673472 & 1.52961617790983 & -0.20558394851320 \\
O & -2.94852461504975 & 2.45120691220805 & -0.87435702512359 \\
O & -2.96312553883362 & 0.70713387243003 & 0.59553923944003 \\
C & -1.59138163928500 & 2.38692246730221 & -0.35968032826208 \\
\hline C & -1.55706016674122 & 0.95653258832325 & 0.32791636470043 \\
C & -1.44233230855613 & 3.54655269526850 & 0.63248076382664 \\
H & -2.13412492423189 & 3.44288784054027 & 1.48165328857140 \\
\hline H & -1.68288772330737 & 4.48809413659086 & 0.11624473516331 \\
\hline H & -0.41668457132016 & 3.61897513149307 & 1.02511602082409 \\
C & -0.62114105813951 & 2.56867009521722 & -1.52443049664392 \\
\hline H & -0.81148781982642 & 1.84753574898000 & -2.33019326696200 \\
\hline H & 0.42238877634948 & 2.45582392257230 & -1.18990649566076 \\
\hline H & -0.73606547097093 & 3.57928140803177 & -1.94517138593348 \\
\hline C & -1.08292148139514 & -0.16137488715867 & -0.60843425888858 \\
\hline H & -1.28379923990664 & -1.13246284318888 & -0.13145595594633 \\
H & 0.26952343769742 & 1.14505752953537 & 1.50150174171030 \\
H & -0.00368697864795 & -0.09256732944200 & -0.81305895960779 \\
H & -1.62162780422932 & -0.14051892291829 & -1.56748531212716 \\
C & -0.79240839538504 & 0.89110936696089 & 1.64783062095000 \\
\hline & -0.84501584795422 & -0.130688868328106 & 2.05369572234379 \\
\hline & -1.21583194378665 & 1.57120924101376 & 2.39864142185461 \\
\hline
\end{tabular}

TS 4s-2a

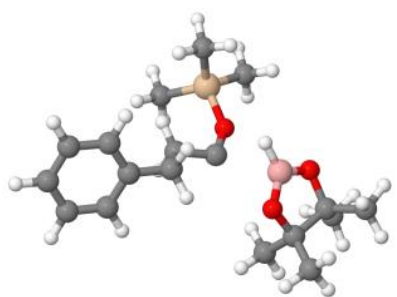

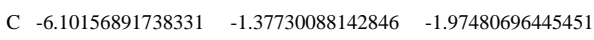

$\begin{array}{llll}\text { C } & -6.38957170793766 & -1.98730747545887 & -0.75027714172435\end{array}$

$\begin{array}{llll}\text { C } & -5.51145551874729 & -1.84489351892318 & 0.32887724280823\end{array}$

$\begin{array}{llll}\text { C } & -4.33148768663760 & -1.09255809081303 & 0.20960760491181\end{array}$

$\begin{array}{llll}\text { C } & -4.05454009348387 & -0.48851354722957 & -1.02878753619685\end{array}$

$\begin{array}{llll}\text { C } & -4.92838569576791 & -0.62758414926171 & -2.11068647757296\end{array}$ 


\begin{tabular}{|c|c|c|c|}
\hline $\mathrm{C}$ & -3.39872606384281 & -0.90754573487527 & 1.38692700526941 \\
\hline $\mathrm{C}$ & -3.68967468704331 & 0.37274530927163 & 2.19406070956820 \\
\hline $\mathrm{H}$ & -2.35049186768640 & -0.87437639784655 & 1.05213698588247 \\
\hline $\mathrm{H}$ & -3.47450877062637 & -1.77606518376504 & 2.06072718140780 \\
\hline $\mathrm{C}$ & -2.61153683341313 & 0.72575156444273 & 3.18787715118430 \\
\hline $\mathrm{H}$ & -4.71795542229289 & 0.35372029191877 & 2.61088025647180 \\
\hline $\mathrm{H}$ & -3.65668042535598 & 1.24103255150710 & 1.50007936453880 \\
\hline $\mathrm{H}$ & -6.78451763921940 & -1.49024697598679 & -2.82116210531635 \\
\hline $\mathrm{H}$ & -7.30023073894692 & -2.58201175453043 & -0.63517948536592 \\
\hline $\mathrm{H}$ & -5.74105664115196 & -2.33376187838935 & 1.28101735539719 \\
\hline $\mathrm{H}$ & -3.13466610423641 & 0.09296031158361 & -1.14754092295139 \\
\hline $\mathrm{H}$ & -4.68929691421480 & -0.15347092916076 & -3.06686975308362 \\
\hline $\mathrm{H}$ & -3.22298499992567 & 2.15668516680389 & 7.12934897311054 \\
\hline $\mathrm{C}$ & -3.82926279279669 & 2.83218652861219 & 6.50440806610096 \\
\hline $\mathrm{H}$ & -4.64511618725005 & 3.23725999665849 & 7.12641062948876 \\
\hline $\mathrm{H}$ & -3.18564059564086 & 3.67201578925297 & 6.19581567721778 \\
\hline $\mathrm{H}$ & -4.89997506833681 & -0.28971739164527 & 6.08161483177264 \\
\hline $\mathrm{Si}$ & -4.50570772562519 & 1.92454362016028 & 5.01252863899597 \\
\hline $\mathrm{C}$ & -5.52374977124895 & 0.43058206995461 & 5.52720948028274 \\
\hline $\mathrm{H}$ & -5.97132275845260 & -0.09425286000230 & 4.66883921971741 \\
\hline $\mathrm{H}$ & -6.34483576833265 & 0.74596265353797 & 6.19392196520403 \\
\hline $\mathrm{C}$ & 12064826 & 3.09568105188762 & $3.8696 \mathrm{C}$ \\
\hline $\mathrm{H}$ & -6.24503673024022 & 3.59734287850595 & 4.42482108960774 \\
\hline $\mathrm{H}$ & -4.76155943106178 & 3.88054926082903 & 3.48435954257580 \\
\hline $\mathrm{H}$ & -5.88416809454679 & 2.57826125198070 & 3.00866483701338 \\
\hline $\mathrm{O}$ & -3.04060608726763 & 1.36830449852866 & 4.23593092154155 \\
\hline B & -0.68248733029423 & -1.26226452170451 & 3.87037702548682 \\
\hline $\mathrm{O}$ & 0.04334728701437 & -0.58482787752505 & 4.80925225851730 \\
\hline $\mathrm{O}$ & 0.00061260439484 & -1.47781630653274 & 2.70409784945657 \\
\hline $\mathrm{C}$ & 1.20512871651985 & -0.67292834906297 & 2.73425954713300 \\
\hline $\mathrm{C}$ & 1.37359688662475 & -0.36106955908732 & 4.28802390156053 \\
\hline $\mathrm{C}$ & 2.30175721648653 & -1.34349937197085 & 5.01460169665342 \\
\hline $\mathrm{H}$ & 2.01756567962271 & -2.38718684132242 & 4.81431940780716 \\
\hline $\mathrm{H}$ & 2.21611325187769 & -1.17539365813565 & 6.09902424610660 \\
\hline $\mathrm{H}$ & 3.35494058391344 & -1.20585781815294 & 4.72491610551285 \\
\hline $\mathrm{C}$ & 1.78223906194693 & 1.07526606714399 & 4.61361816184150 \\
\hline $\mathrm{H}$ & 1.04745349783182 & 1.79732196091873 & 4.23440750831990 \\
\hline $\mathrm{H}$ & 2.76954696883323 & 1.31484522227930 & 4.18719054570903 \\
\hline $\mathrm{H}$ & 1.84364976134791 & 1.19940298916309 & 5.70595076539021 \\
\hline $\mathrm{C}$ & 0.93488896538881 & 0.57487208326254 & 1.88476417851316 \\
\hline $\mathrm{H}$ & 0.70075134413817 & 0.25762370791945 & 0.85644685921483 \\
\hline $\mathrm{H}$ & 0.06664138174672 & 1.12875816643405 & 2.26990571675117 \\
\hline $\mathrm{H}$ & 1.81097372207374 & 1.24037107772885 & 1.84588323592112 \\
\hline $\mathrm{C}$ & 2.34070557776554 & -1.48764988288110 & 2.11549823263495 \\
\hline & & -2.45 & 00603 \\
\hline
\end{tabular}

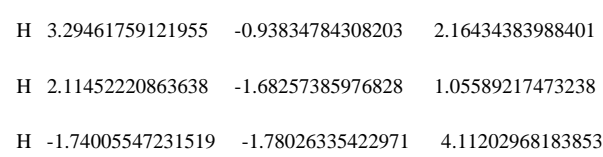

2a

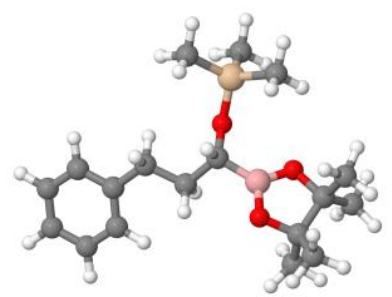

C $\quad-6.31578350979150 \quad-1.34407785893175 \quad-2.33142200178556$

$\begin{array}{llll}\text { C } & -4.97703898921518 & -0.97485229450627 & -2.18998889686091\end{array}$

$\begin{array}{llll}\text { C } & -4.39947081713045 & -0.87545886192104 & -0.91781821534463\end{array}$

$\begin{array}{llll}\text { C } & -5.14689412093054 & -1.14269401152269 & 0.23966198532792\end{array}$

$\begin{array}{llll}\text { C } & -6.49589940006918 & -1.51068358746165 & 0.07849890300861\end{array}$

C $-7.07561508623642 \quad-1.61266704113392 \quad-1.18672970795674$

$\begin{array}{llll}\text { C } & -4.56489615018671 & -1.06743791430847 & 1.64074335419629\end{array}$

$\begin{array}{llll}\text { C } & -3.17261261991171 & -0.45050643631436 & 1.77977735890881\end{array}$

$\begin{array}{llll}\text { H } & -4.54429163331457 & -2.09004932909831 & 2.06504656039409\end{array}$

$\begin{array}{llll}\text { H } & -5.26092473505838 & -0.49929936351497 & 2.27998870441045\end{array}$

C $\quad-2.69492731596643 \quad-0.34440437720030 \quad 3.24012056978965$

$\begin{array}{llll}\text { H } & -3.17160939533753 & 0.56252198685351 & 1.33900645766091\end{array}$

H $\quad-2.42766529615475 \quad-1.04192500010165 \quad 1.22200527116007$

$\begin{array}{llll}\text { H } & -6.76684666362988 & -1.42078343647888 & -3.32447540491648\end{array}$

$\begin{array}{llll}\text { H } & -4.37169199697378 & -0.76003965066615 & -3.07535226417700\end{array}$

$\begin{array}{llll}\text { H } & -3.35018733807499 & -0.58485406090049 & -0.83627353091413\end{array}$

H $\quad-7.10020643464353 \quad-1.71926883337169 \quad 0.96731590110741$

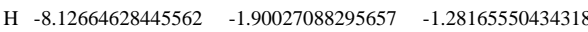

$\begin{array}{llll}\text { H } & -2.09509302711247 & -0.75188355222541 & 6.54811682337005\end{array}$

$\begin{array}{llll}\text { C } & -2.65315711843478 & 0.18053400895126 & 6.73834471158521\end{array}$

$\begin{array}{llll}\text { H } & -2.99796374818901 & 0.15085349098380 & 7.78617868750340\end{array}$

$\begin{array}{llll}\text { H } & -1.95039999166425 & 1.02087739497378 & 6.62532425985875\end{array}$

H $\quad-4.74744747125231 \quad-2.05685447552387 \quad 5.46048351067949$

Si $-4.10981349804671 \quad 0.37534623351690 \quad 5.55380823987507$

C $\quad-5.25955245719865 \quad-1.11557644432271 \quad 5.72302677631465$

$\begin{array}{llll}\text { H } & -6.13758311480258 & -1.01682063417380 & 5.06320025329142\end{array}$

$\begin{array}{llll}\text { H } & -5.62619207052728 & -1.21817494767345 & 6.75889231658478\end{array}$

$\begin{array}{llll}\text { C } & -5.04030937869054 & 1.97291447423854 & 5.88737883817212\end{array}$

$\begin{array}{llll}\mathrm{H} & -5.46881348373964 & 1.98315705055204 & 6.90376959093072\end{array}$

$\begin{array}{llll}\text { H } & -4.36938922779421 & 2.84204888238553 & 5.78992760969457\end{array}$

$\begin{array}{llll}\text { H } & -5.86614416197833 & 2.10343873498579 & 5.16887664535490\end{array}$

$\begin{array}{llll}\text { O } & -3.58105376678879 & 0.50578786251876 & 3.96332992384455\end{array}$ 

B $-1.18788100720049 \quad 0.15310131870900 \quad 3.30377495152369$
$\begin{array}{llll}\text { H } & -2.71687335537235 & -1.37535935867457 & 3.66109912085706\end{array}$
$\begin{array}{llll}\text { O } & -0.74173768387353 & 1.22467665008806 & 4.02333256610630\end{array}$
$\begin{array}{llll}\text { O } & -0.18761475064265 & -0.47603403547207 & 2.60928371308108\end{array}$
$\begin{array}{llll}\text { C } & 1.00651224333268 & 0.34393098635243 & 2.68840611810910\end{array}$
$\begin{array}{llll}\text { C } & 0.70844901148203 & 1.24881319023650 & 3.95850210940397\end{array}$
$\begin{array}{llll}\text { C } & 1.22597303934467 & 0.64517217209598 & 5.26971710613438\end{array}$

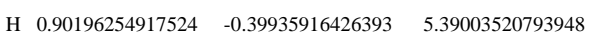
$\begin{array}{llll}\text { H } & 0.82104116383588 & 1.22218755192418 & 6.11456381375402\end{array}$
H $\quad 2.32461933178645 \quad 0.67752971768512 \quad 5.32612678519094$
C $\quad 1.16296940453747 \quad 2.70183743268940 \quad 3.83940957929292$
$\begin{array}{llll}\text { H } & 0.68382584832405 & 3.21085130369280 & 2.99278566995583\end{array}$
$\begin{array}{llll}\text { H } & 2.25596963716371 & 2.76440397369855 & 3.71641857457045\end{array}$
$\begin{array}{llll}\text { H } & 0.89185008316368 & 3.24827143390441 & 4.75561468071571\end{array}$
$\begin{array}{llll}\text { C } & 1.08654272843729 & 1.13869187397754 & 1.37897698338026\end{array}$
$\begin{array}{llll}\text { H } & 1.09073361593536 & 0.43301653243697 & 0.53451672408293\end{array}$
$\begin{array}{llll}\text { H } & 0.21924517285568 & 1.80472754895301 & 1.25718493708884\end{array}$

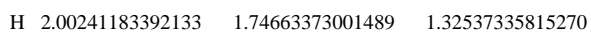
$\begin{array}{llll}\text { C } & 2.22034819032720 & -0.57374234020569 & 2.81518075502332\end{array}$
H $2.12044896384231 \quad-1.26886824105918 \quad 3.65926313701521$
$\begin{array}{llll}\text { H } & 3.14470378024102 & 0.01075711557513 & 2.94750866889560\end{array}$
$\begin{array}{llll}\text { H } & 2.32558050268368 & -1.17302651801002 & 1.89787771300094\end{array}$

\section{T - Spindensity}

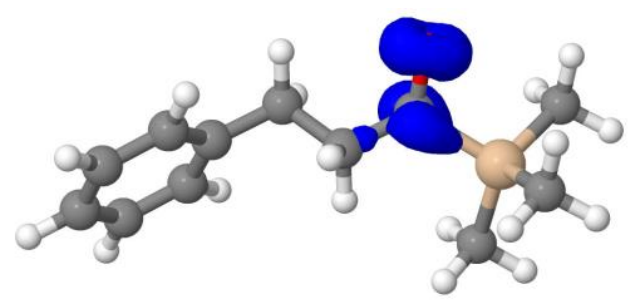

TS 3-4 - Spindensity

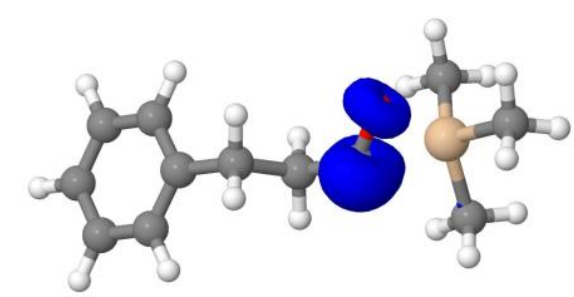

4S - Spindensity

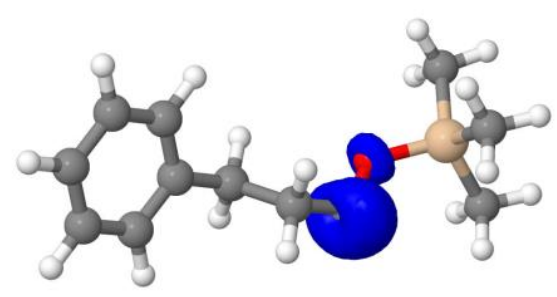

\section{S - Spindensity}

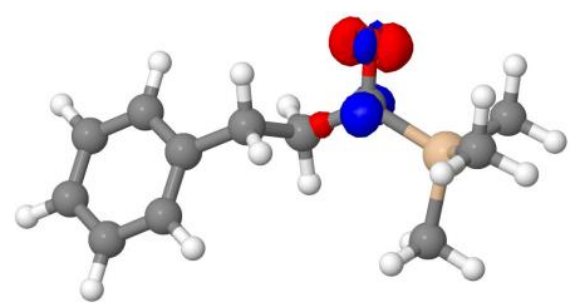

\subsection{Further Investigation of Pathway towards Intermediate X}

To further investigate the pathway towards Intermediate $\mathbf{X}$, a relaxed surface scan was conducted. The geometries were optimized using the B3LYP hybrid functional ${ }^{[5]}$ and Ahlrichs' def2-SVP double-zeta basis set ${ }^{[6]}$. Here, the $\mathrm{B}-\mathrm{H}$ bond distance is analyzed and scanned between 1.0 and $3.0 \AA$. The result is 
presented in Figure S7. The minimum at $2.2 \AA$ can be assigned to product $\mathbf{2 a}$. No further minimum in the scanned region could be found and renders $\mathbf{X}$ an unlikely intermediate.

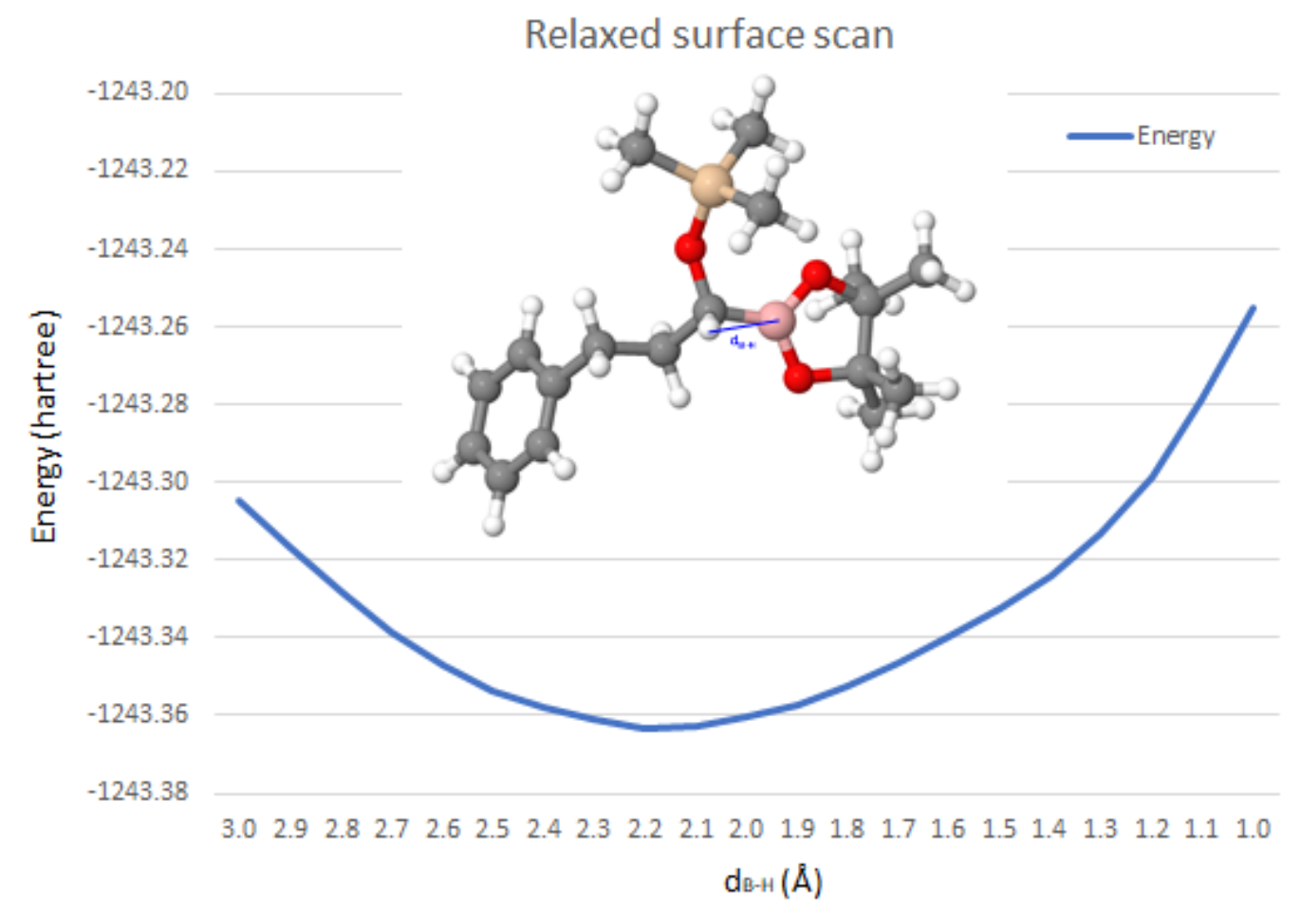

Figure S7: Energy as a functional of reaction coordinate. Scan ran from point $3.0 \AA$ to $1.0 \AA$. 


\subsection{Characterisation of Excited Acylsilane}

Acylsilane 1a and its vertically excited state $1 \mathbf{a}^{*}$ were analyzed via TDDFT calculations (first ten excited states). With this, an absorption band at $357.8 \mathrm{~nm}$, which corresponds to an excitation from the HOMO (MO55) to the LUMO (MO56), could be computed. Further absorption bands where detected at $245.5 \mathrm{~nm}, 240.2 \mathrm{~nm}$ and $234.9 \mathrm{~nm}$. The observed excitation can be interpreted as a carbonylcentered $\mathrm{n}_{\mathrm{C}-\mathrm{O}}->\pi_{\mathrm{C}-\mathrm{O}}^{*}$ transition. The HOMO shows significant contributions from the $\sigma_{\mathrm{C}-\mathrm{Si}}$ orbital, which accounts for its higher energy and the low HOMO-LUMO gap compared to classical carbonyl compounds. 
MO54

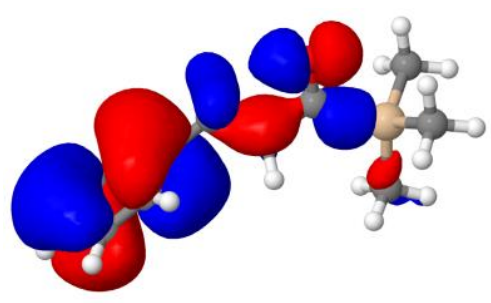

MO55

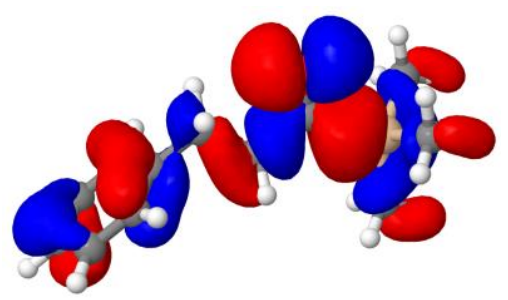

MO56

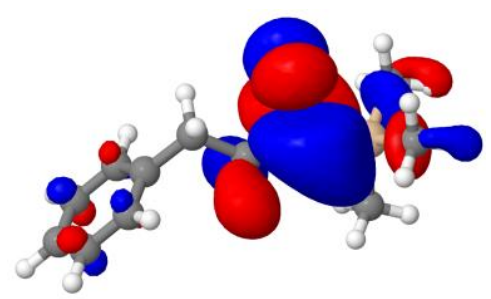

MO57

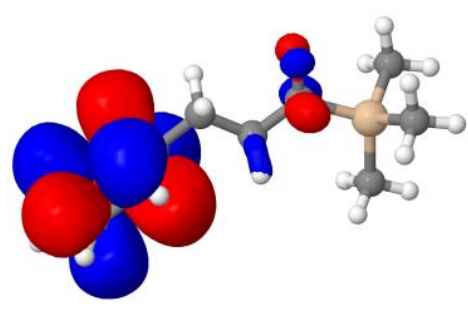

MO58

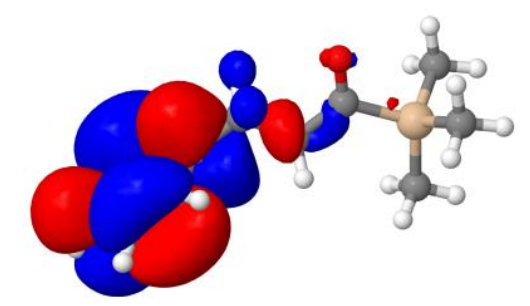

$$
\begin{array}{ll}
53 \text {-> } 57: & 0.167834 \\
54 \text {-> } 58: & 0.197156
\end{array}
$$$$
55 \text {-> } 58: 0.108510
$$

STATE 1: $2.801 \mathrm{eV}, 442.6 \mathrm{~nm}, \mathrm{f}=$ 0.000000000

$$
\begin{aligned}
& 54 \text {-> } 56: 0.041058 \\
& 55 \text {-> } 56: 0.443866
\end{aligned}
$$

STATE 2: $3.466 \mathrm{eV}, 357.8 \mathrm{~nm}, \mathrm{f}=$ 0.003652152

$$
\begin{aligned}
& 54 \text {-> } 56: 0.028843 \\
& 55 \text {-> } 56: 0.458567
\end{aligned}
$$

STATE 3: $4.043 \mathrm{eV}, 306.7 \mathrm{~nm}, \mathrm{f}=$ 0.000000000

53 -> $56: 0.012183$
STATE 4: $4.512 \mathrm{eV}, 274.8 \mathrm{~nm}, \mathrm{f}=$ 0.000000000

$$
\begin{array}{ll}
53 \text {-> } 58: & 0.053823 \\
54 \text {-> } 56: & 0.041701 \\
54 \text {-> } 57: & 0.197618 \\
55 \text {-> } 57: & 0.194841
\end{array}
$$

STATE 5: $4.603 \mathrm{eV}, 269.4 \mathrm{~nm}, \mathrm{f}=$ 0.00000000

$$
\begin{array}{ll}
53 \text {-> } 56: 0.034352 \\
53 \text {-> } 57: 0.275040
\end{array}
$$




$$
\begin{aligned}
& 54 \text {-> } 58: 0.092243 \\
& 53 \text {-> } 56: 0.075504 \\
& 55 \text {-> } 58: 0.095135 \\
& 54 \text {-> } 58: 0.010128 \\
& 55 \text {-> } 58: 0.409722
\end{aligned}
$$

STATE 6: $4.991 \mathrm{eV}, 248.4 \mathrm{~nm}, \mathrm{f}=$ 0.000000000

$$
\begin{array}{ll}
53 \text {-> } 58: & 0.418135 \\
54 \text {-> } 56: & 0.040296 \\
55 \text {-> } 57: & 0.028741
\end{array}
$$

STATE 7: $\quad 5.050 \mathrm{eV}, 245.5 \mathrm{~nm}, \mathrm{f}=$ 0.002929755

$$
\begin{array}{lll}
53 \text {-> } 58: & 0.060736 \\
54 \text {-> } 56: & 0.260370 \\
55 \text {-> } 56: & 0.027501 \\
55 \text {-> } 57: & 0.139652
\end{array}
$$

STATE 8: $5.114 \mathrm{eV}, 242.5 \mathrm{~nm}, \mathrm{f}=$ 0.000000000

$$
\begin{array}{lll}
53 \text {-> } 58: & 0.016871 \\
54 \text {-> } 56: & 0.368453 \\
54 \text {-> } 57: & 0.014995 \\
55 \text {-> } 56: & 0.026399 \\
55 \text {-> } 57: & 0.068502
\end{array}
$$

STATE 9: $5.162 \mathrm{eV}, 240.2 \mathrm{~nm}, \mathrm{f}=$ 0.000327718

$$
\begin{array}{lll}
53 \text {-> } 58: & 0.034092 \\
54 \text {-> } 56: & 0.201213 \\
54 \text {-> } 57: & 0.011763 \\
55 \text {-> } 57: & 0.246289
\end{array}
$$

STATE 10: $5.278 \mathrm{eV}, 234.9 \mathrm{~nm}, \mathrm{f}=$ $\mathbf{0 . 1 0 7 4 5 8 8 0 2}$ 


\section{References}

[1] (a) Gasking, D. I.; Whitham, G. H. Some Bifunctional Acylsilanes and Their Photochemical Reactions. J. Chem. Soc. Perkin Trans. 1, 1985, 409-413. (b) Ricci, A.; Degl'Innocenti, A.; Borselli, G.; Reginato, G. Synthesis of Polyfunctionalized Acylsilanes via Propenoyltrimethylsilane. Tetrahedron Lett. 1987, 28, 4093-4096. (c) Linderman, R. J.; Suhr, Y. Synthesis of ( $\alpha$ Hydroxyalky1)silanes from Formyltrimethylsilane. A New Route to Acetylenic Acylsilanes. J. Org. Chem. 1988, 53, 1569-1572. (d) Suda, K.; Watanabe, J.-i.; Takanami, T. Anodic Oxidation of 2alkyl-2-trialkylsilyl-1,3-dithianes a Facile Preparation of Acylsilanes. Tetrahedron Lett. 1992, 33, 1355-1356. (e) Yang, S.-Y.; Jiaang, W.-T.; Cherng, C.-D.; Tang, K.-H.; Huang, C.-H.; Tsai, Y-M. The Scope and Limitations of Intramolecular Radical Cyclizations of Acylsilanes with Alkyl, Aryl, and Vinyl Radicals. J. Org. Chem. 1997, 62, 9089-9098. (f) Tsai, Y.-M.; Cherng, C.-D.; Nieh, H.C.; Sieh, J.-A. The Formation of Oxygen-Containing Heterocycles via Intramolecular Cyclizations of Halo-substituted Acylsilanes and Unsaturated Acylsilanes. Tetrahedron 1999, 55, 14587-14598. (g) Linghu, X.; Nicewicz, D. A.; Johnson, J. S. Tandem Carbon-Carbon Bond Constructions via Catalyzed Cyanation/Brook Rearrangement/C-Acylation Reactions of Acylsilanes. Org. Lett. 2002, 4, 2957-2960. (h) A synthesis of multisubstituted vinylsilanes via ynolates: stereoselective formation of $\beta$-silyl- $\beta$-lactones followed by decarboxylation. Chem. Commun. 2005, 2477-2479. (i) Ito, K.; Tamashima, H.; Iwasawa, N.; Kusama, H. Photochemically Promoted Transition MetalFree Cross-Coupling of Acylsilanes with Organoboronic Esters. J. Am. Chem. Soc. 2011, 133, 3716-3719. (j) Yu, C.-J.; Gu, P. Intermolecular Schmidt Reaction of Alkyl Azides with Acyl Ailanes. Tetrahedron Lett. 2016, 57, 3568-3570. (k) Deng, Y.; Liu, Q.; Smith, A. B. Oxidative [1,2]-Brook Rearrangements Exploiting Single-Electron Transfer: Photoredox-Catalyzed Alkylations and Arylations. J. Am. Chem. Soc. 2017, 139, 9487-9490.

[2] Neese, F. The ORCA program system. WIREs Comput. Mol. Sci. 2012, 2, 73-78.

[3] Neese, F. Software update: the ORCA program system, version 4.0.WIREs Comput. Mol. Sci. 2017, $8, \mathrm{e} 1327$.

[4] Jmol: an open-source Java viewer for chemical structures in 3D. http://www.jmol.org/

[5] Stephens, P. J.; Devlin, F. J.; Chabalowski, C. F., Frisch, M. J. Ab Initio Calculation of Vibrational Absorption and Circular Dichroism Spectra Using Density Functional Force Fields. J. Phys. Chem. 1994, 98, 11623-11627.

[6] Weigend, F.; Ahlrichs, R. Balanced basis sets of split valence, triple zeta valence and quadruple zeta valence quality for $\mathrm{H}$ to Rn: Design and assessment of accuracy. Phys. Chem. Chem. Phys. 2005, 7, 3297-3305.

[7] Zhao, Y.; Truhlar, D. G. The M06 suite of density functionals for main group thermochemistry, thermochemical kinetics, noncovalent interactions, excited states, and transition elements: two new functionals and systematic testing of four M06-class functionals and 12 other functionals. Theor. Chem. Acc. 2008, 120, 215-241.

[8] Grimme, S. Semiempirical hybrid density functional with perturbative second-order Correlation. $J$. Chem. Phys. 2006, 124, 034108. 


\section{Spectral data}

舟

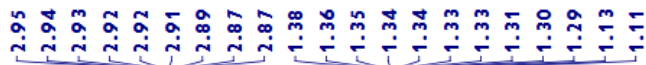<smiles>O=C(CCc1ccccc1)C(F)(F)F</smiles>

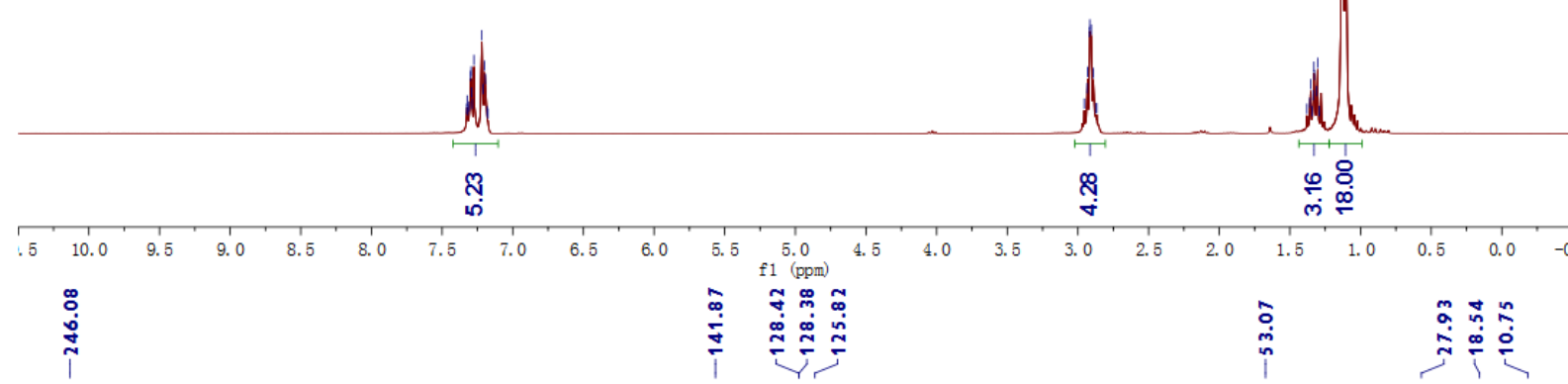<smiles>O=C(CCc1ccccc1)C(F)(F)C(F)(F)F</smiles>

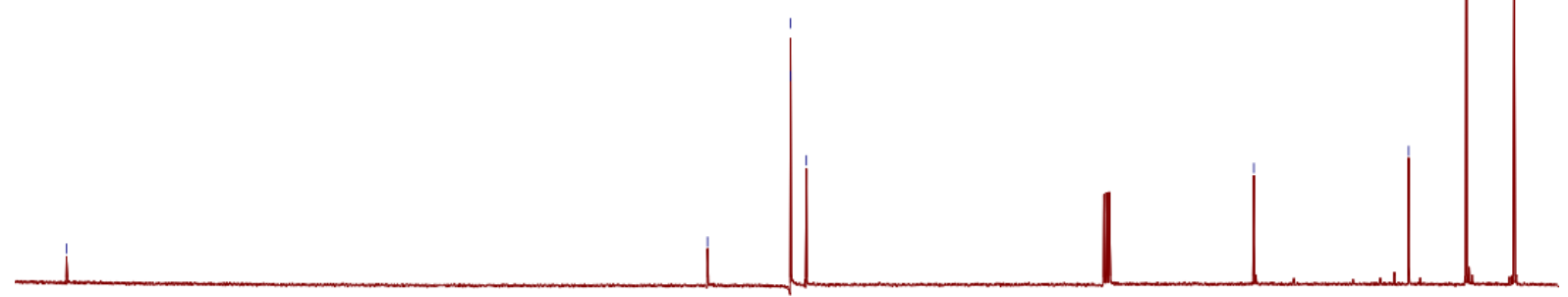

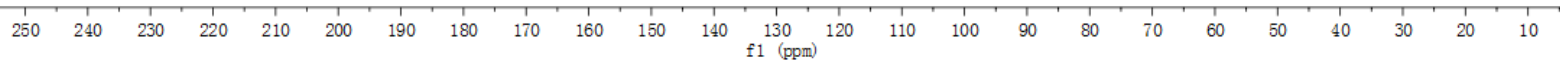




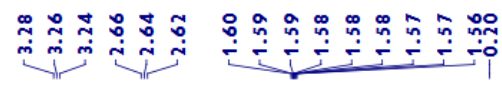

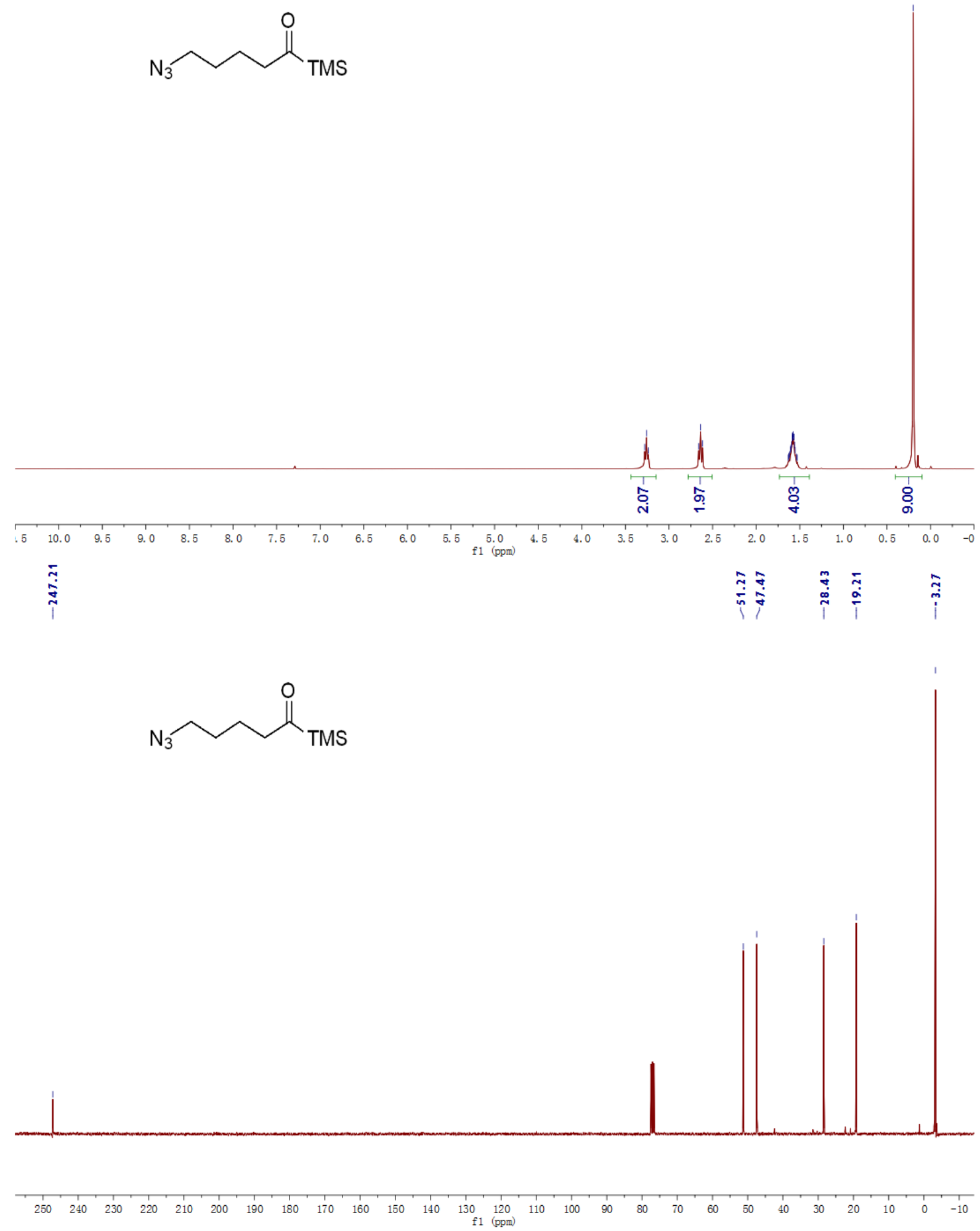



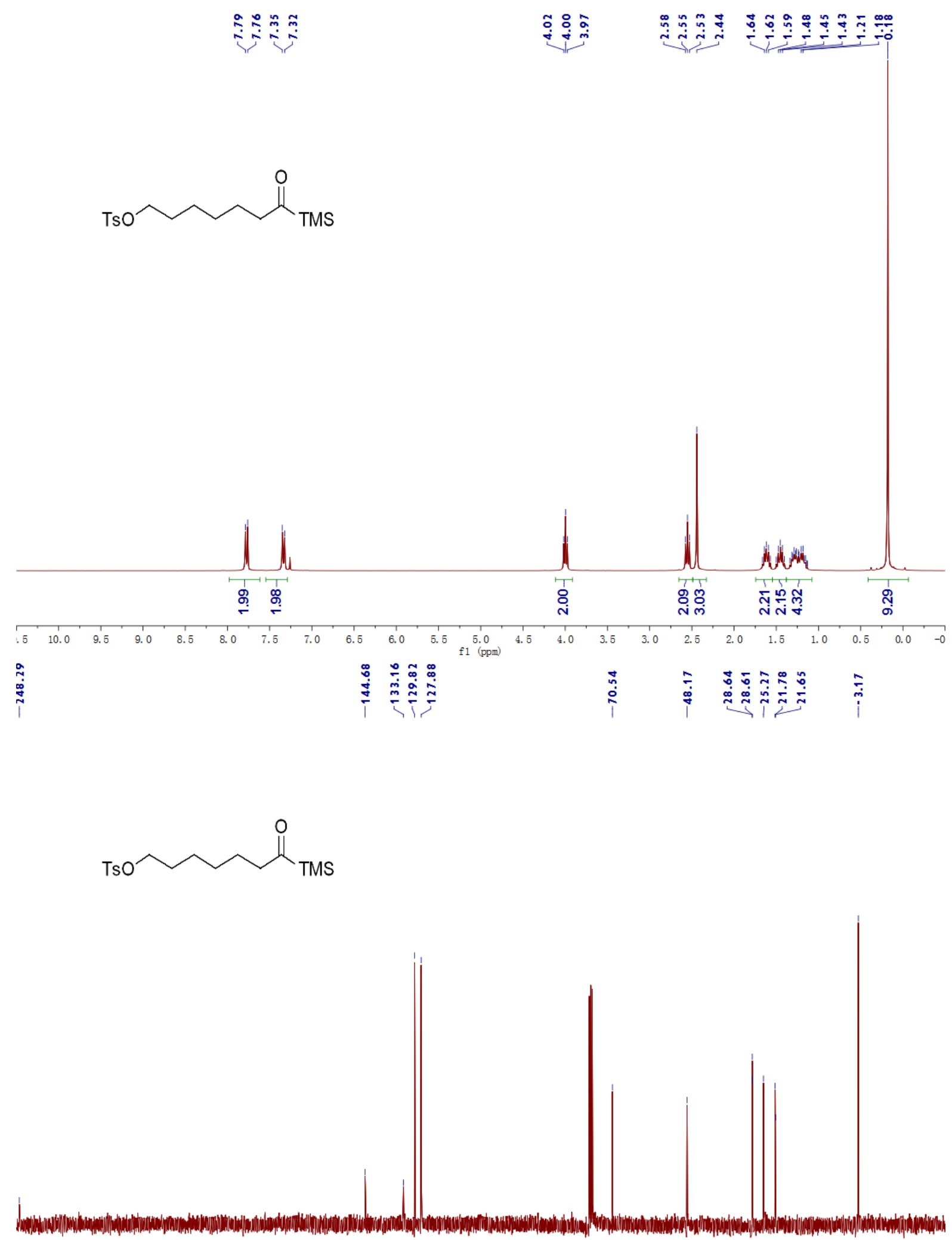

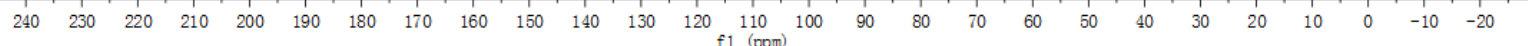


<smiles>CC(=O)CCCCN1C(=O)c2ccccc2C1=O</smiles>

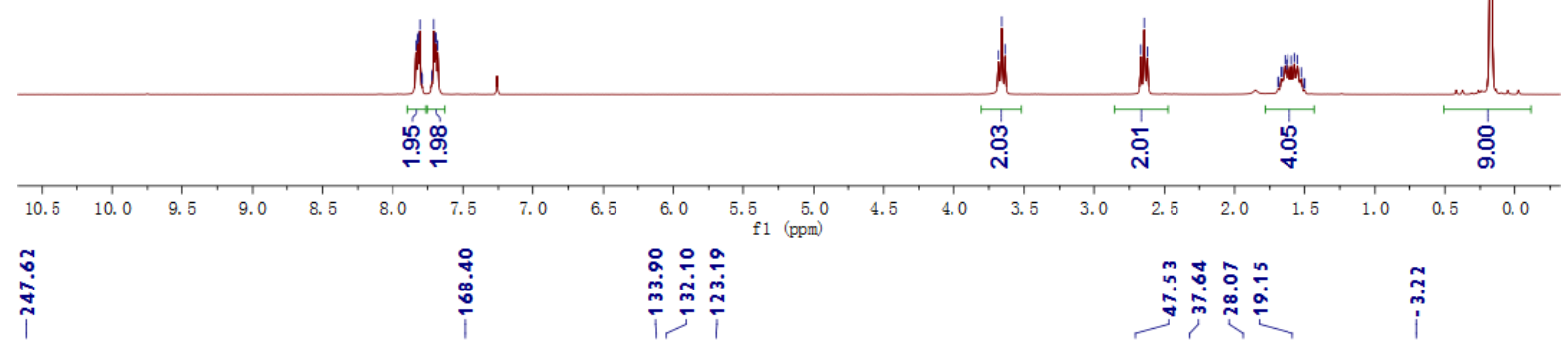<smiles>CS(=O)(=O)CCCCN1C(=O)c2ccccc2C1=O</smiles>

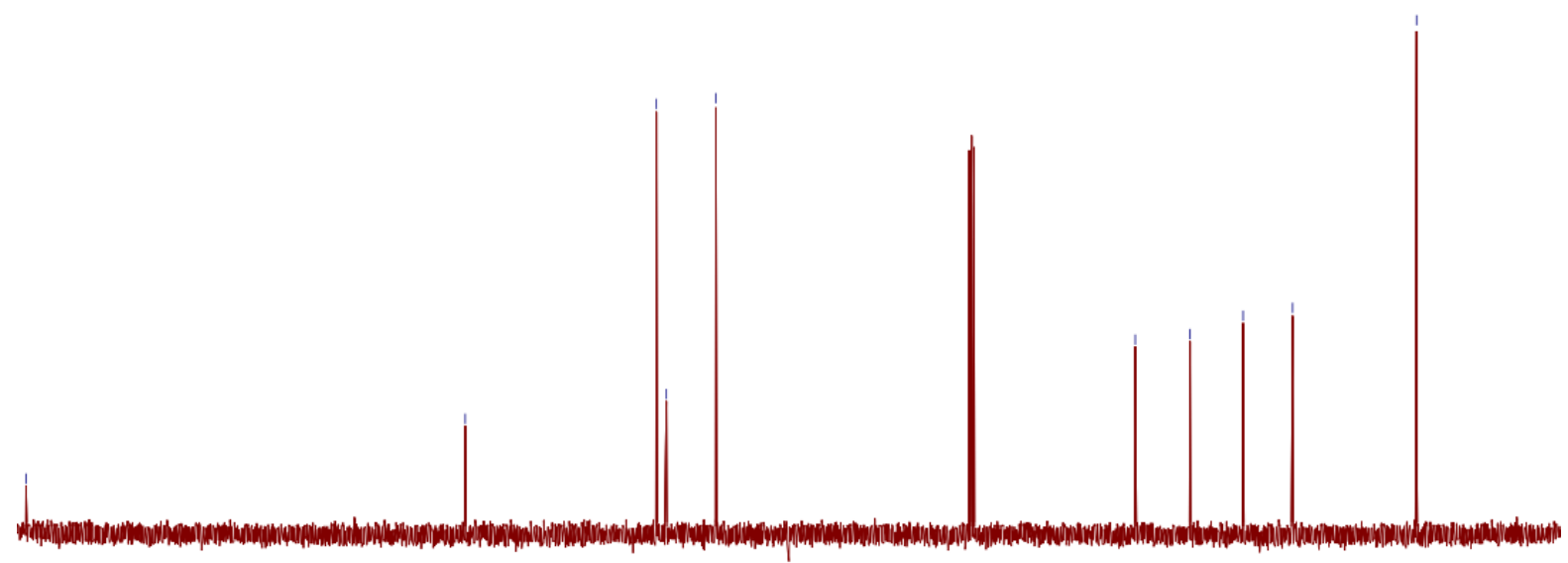

$\begin{array}{llllllllllllllllllllllllllllllllll}240 & 230 & 220 & 210 & 200 & 190 & 180 & 170 & 160 & 150 & 140 & 130 & 120 & 110 & 100 & 90 & 80 & 70 & 60 & 50 & 40 & 30 & 20 & 10 & 0 & -10 & -20\end{array}$ 
<smiles>CC(=O)CCCCN1C(=O)c2ccccc2S1(=O)=O</smiles>

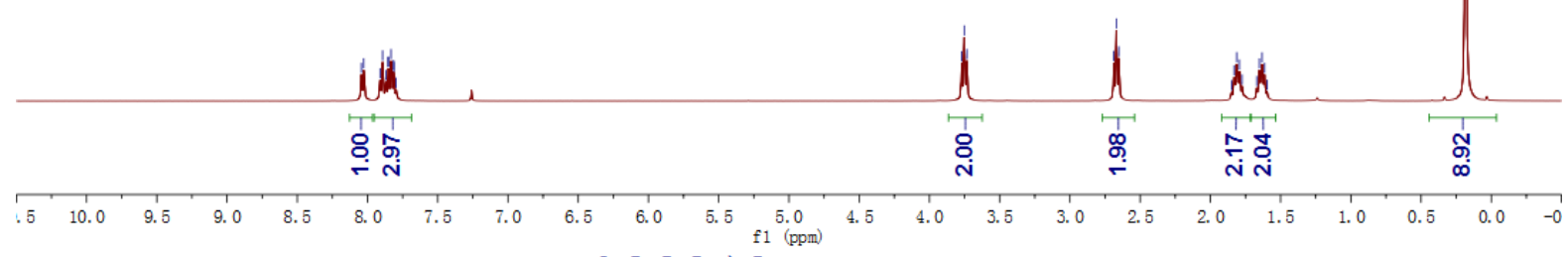

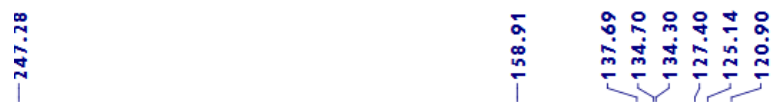

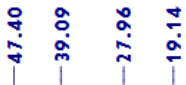<smiles>CS(=O)(=O)C(=O)CCCCN1C(=O)c2ccccc2S1(=O)=O</smiles>

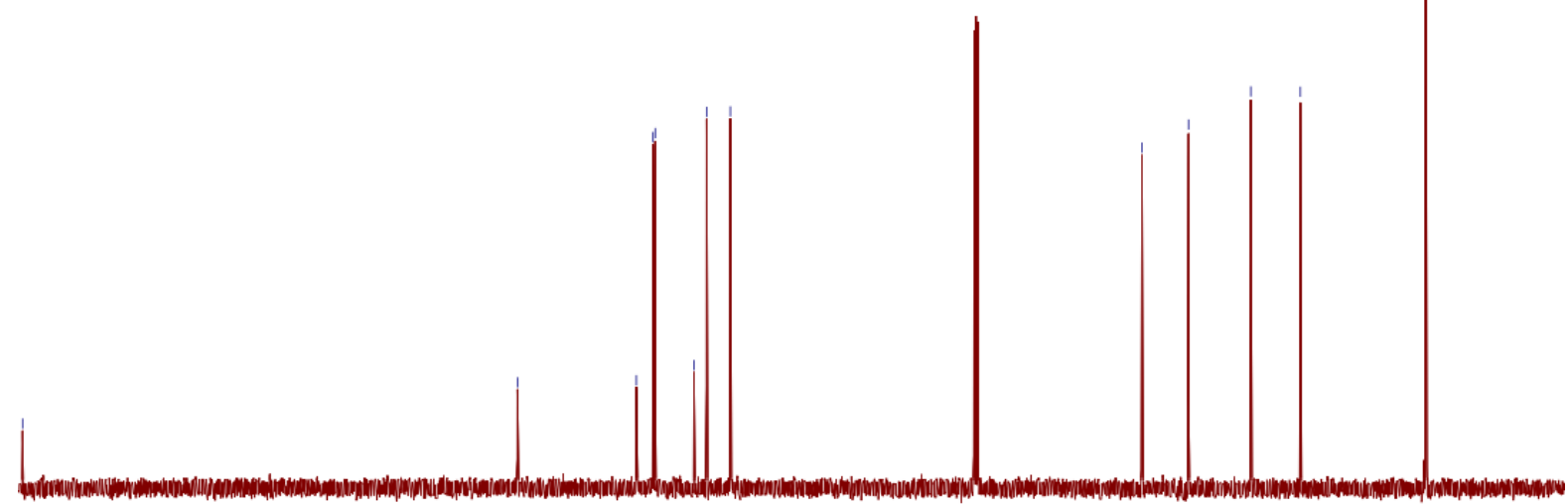

$\begin{array}{llllllllllllllllllllllllllllll}240 & 230 & 220 & 210 & 200 & 190 & 180 & 170 & 160 & 150 & 140 & 130 & 120 & 110 & 100 & 90 & 80 & 70 & 60 & 50 & 40 & 30 & 20 & 10 & 0 & -10 & -20\end{array}$ 

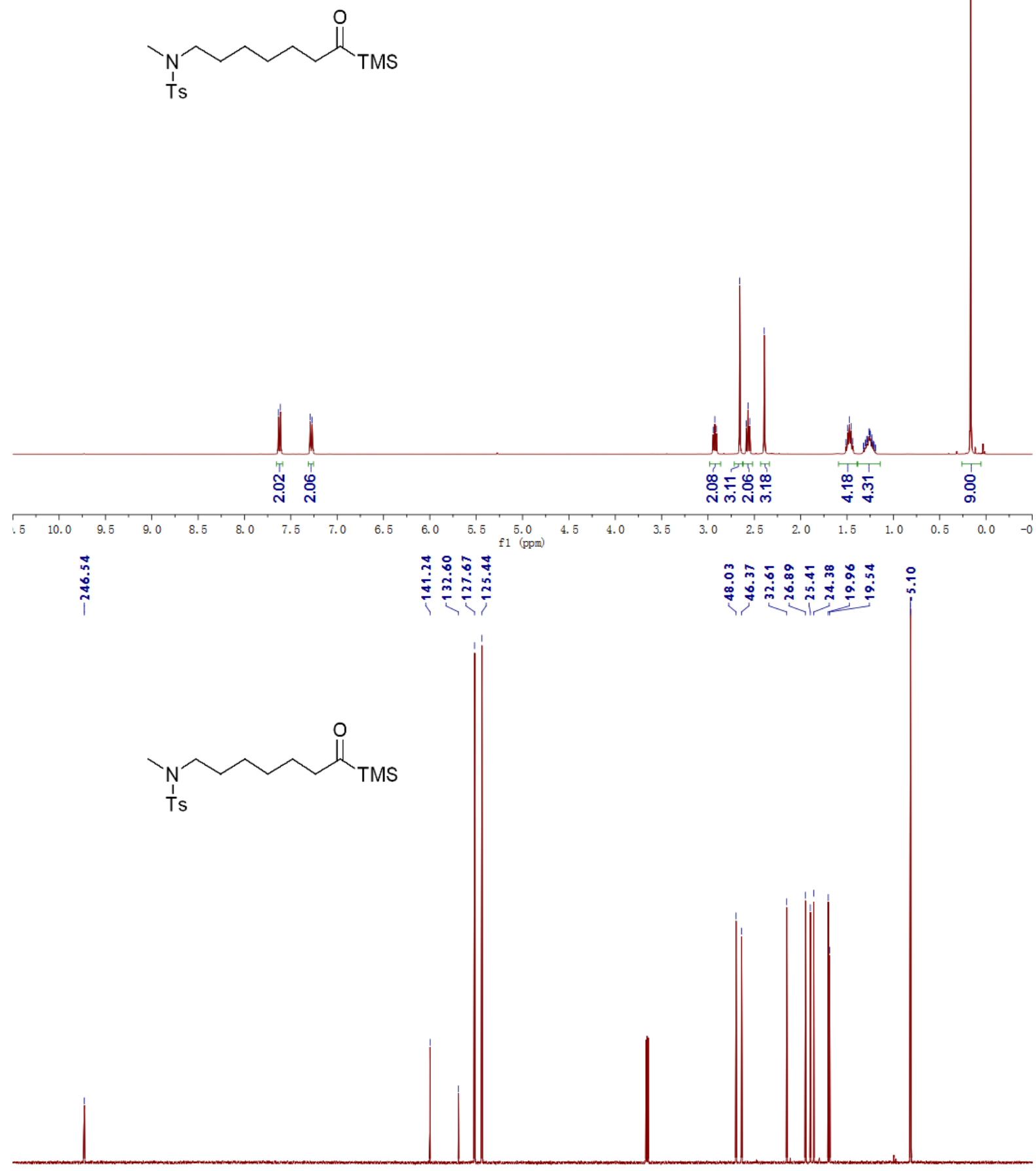

$\begin{array}{llllllllllllllllllllllllllllllllllllll}260 & 250 & 240 & 230 & 220 & 210 & 200 & 190 & 180 & 170 & 160 & 150 & 140 & 130 & 120 & 110 & 100 & 90 & 80 & 70 & 60 & 50 & 40 & 30 & 20 & 10 & 0 & -10 & -20 & -30 & -4\end{array}$ 


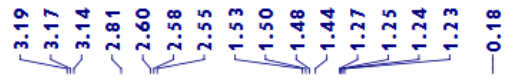

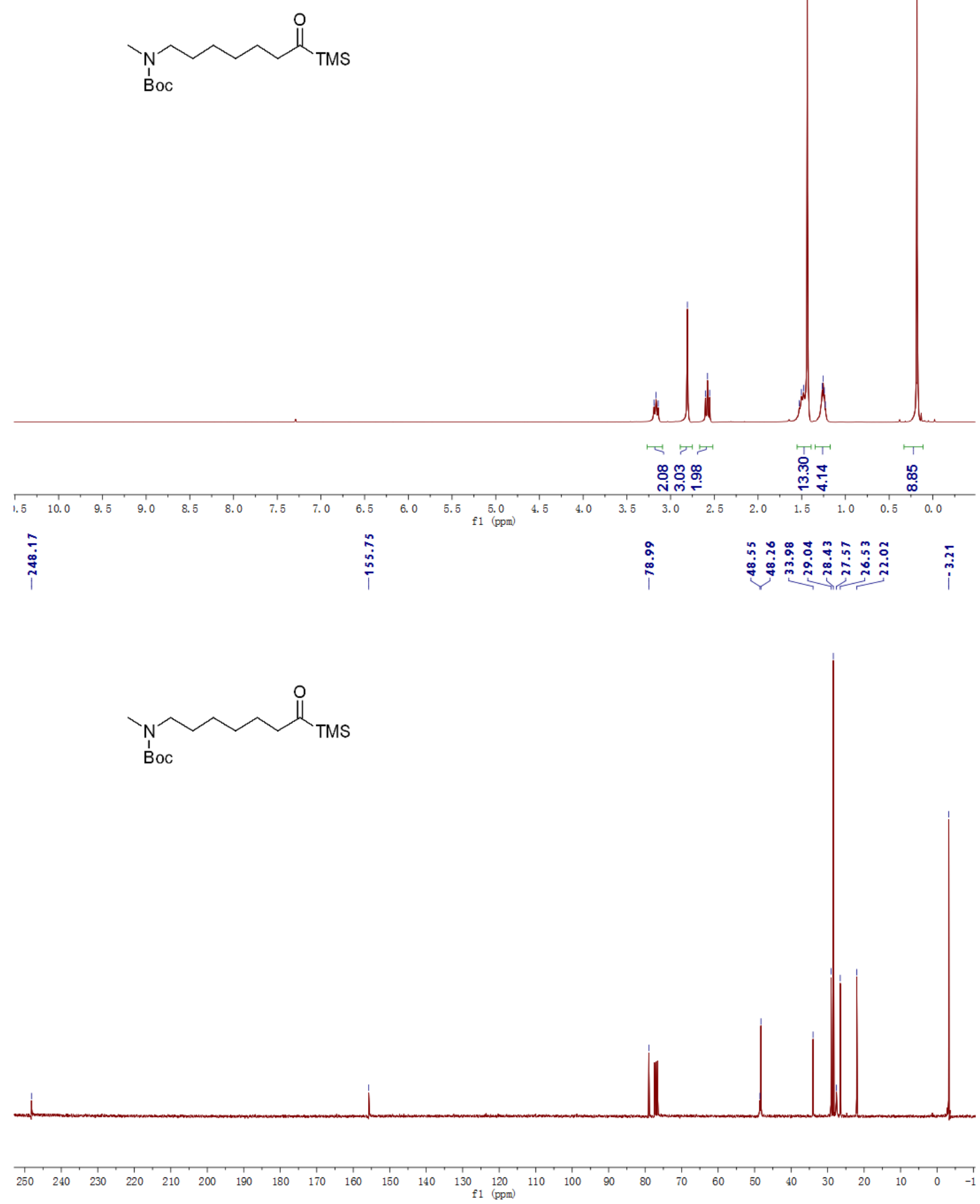




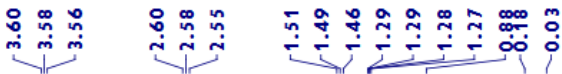

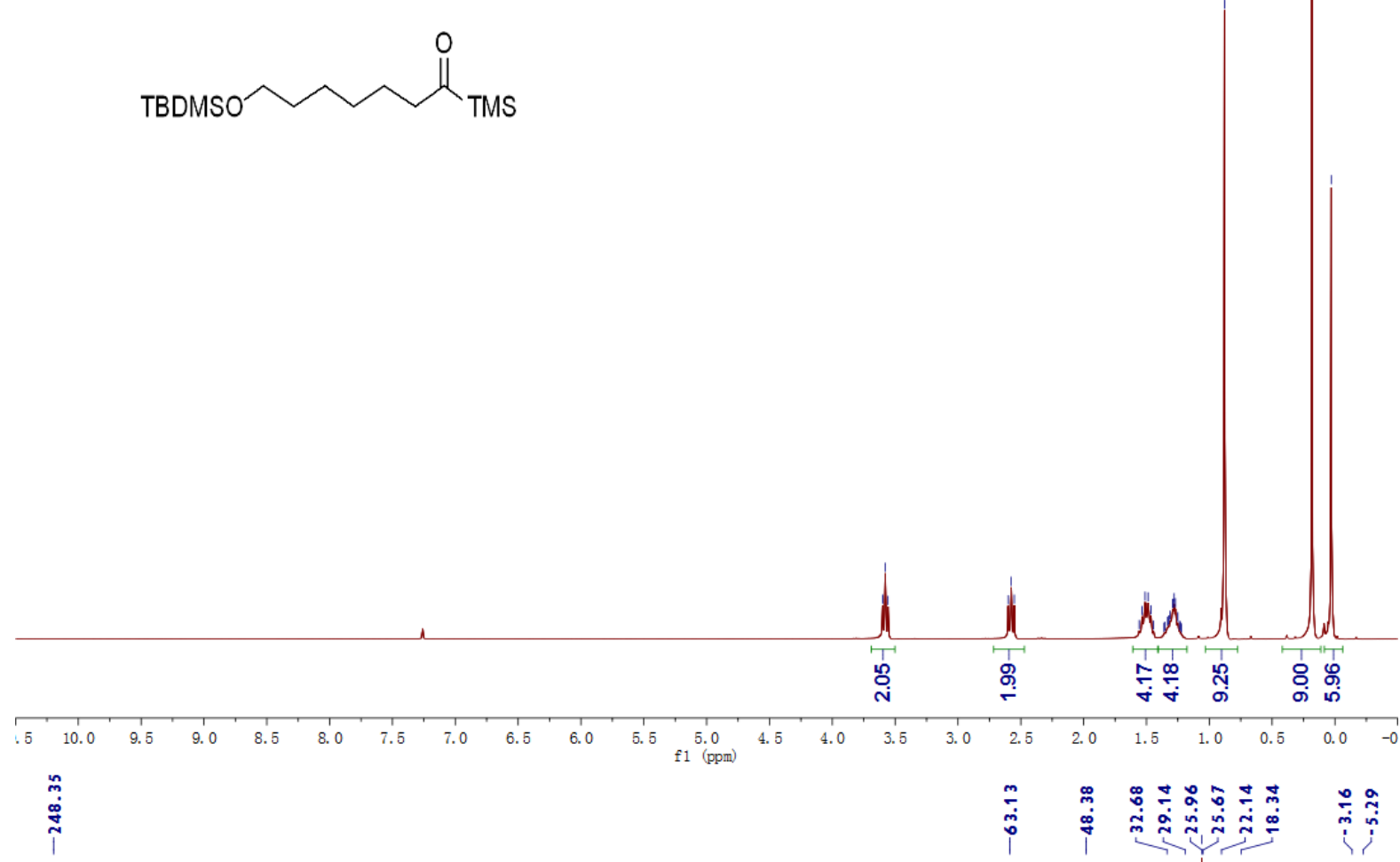

TBDMSO $\sim$ IIMS $_{T}^{O}$ 


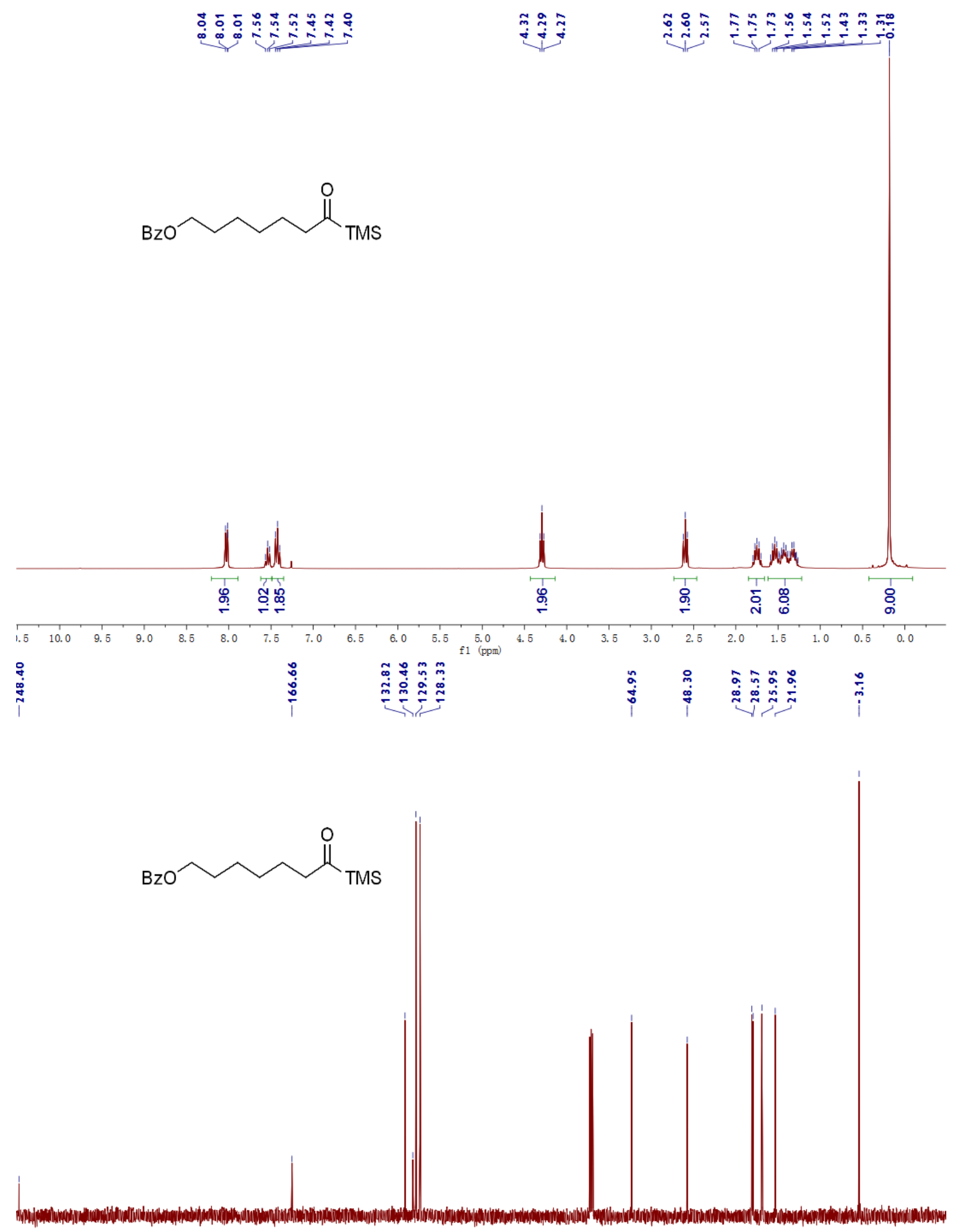

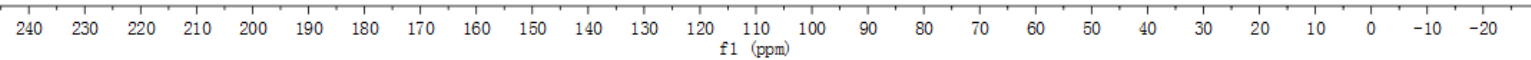




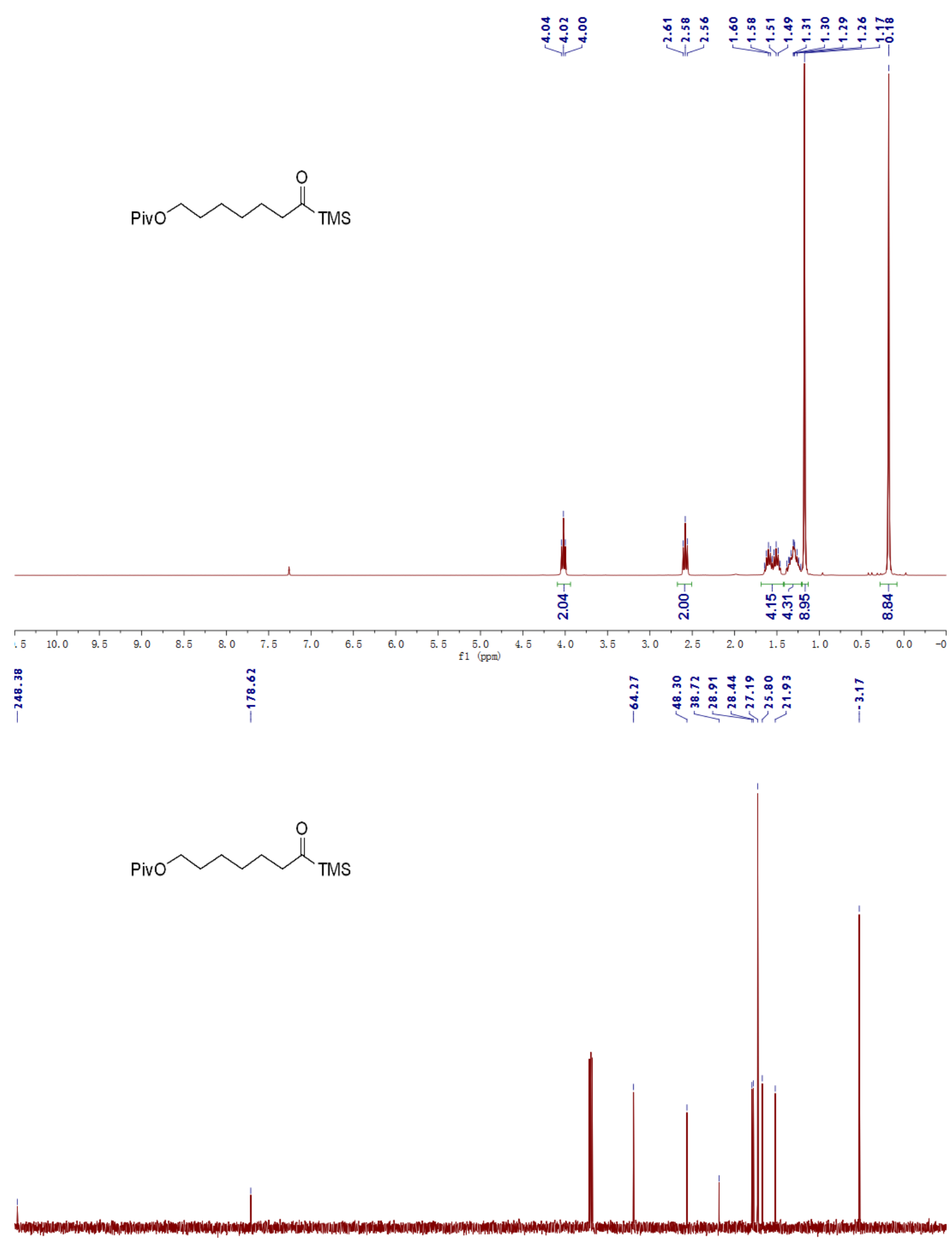

$\begin{array}{llllllllllllllllllllllllllll}240 & 230 & 220 & 210 & 200 & 190 & 180 & 170 & 160 & 150 & 140 & 130 & 120 & 110 & 100 & 90 & 80 & 70 & 60 & 50 & 40 & 30 & 20 & 10 & 0 & -10 & -20 & 1\end{array}$ 
<smiles>CS(=O)(=O)CCCCCCCOC(=O)c1ccco1</smiles>

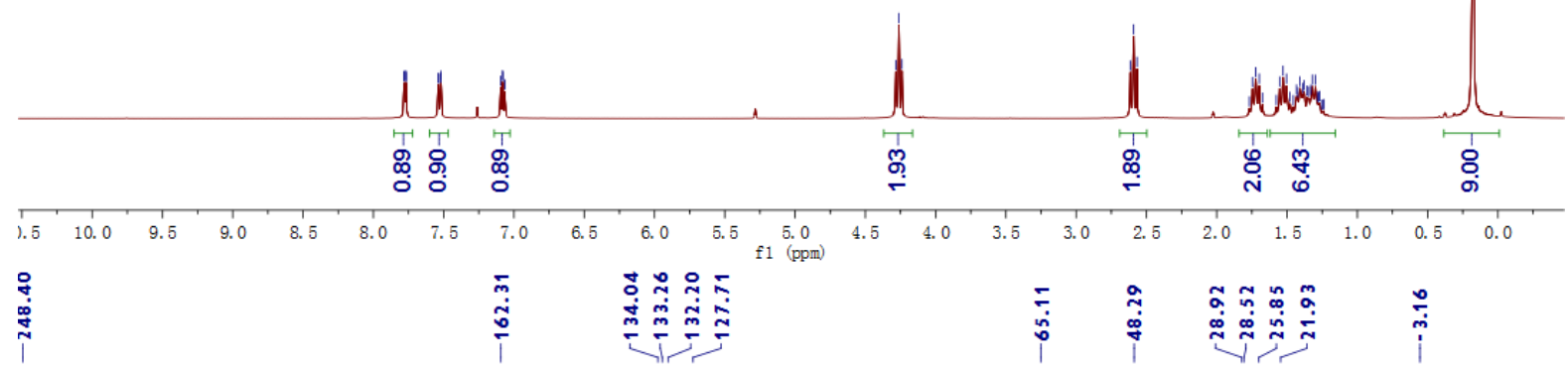<smiles>CS(=O)(=O)CCCCCCCOC(=O)c1ccco1</smiles>

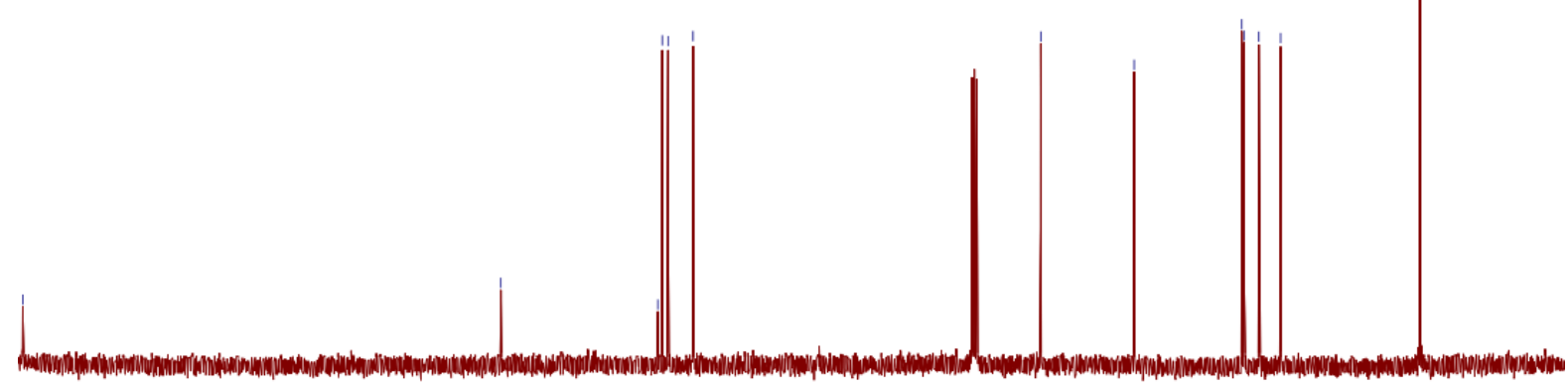

$\begin{array}{lllllllllllllllllllllllllll}240 & 230 & 220 & 210 & 200 & 190 & 180 & 170 & 160 & 150 & 140 & 130 & 120 & \begin{array}{c}110 \\ \mathrm{f} 1\end{array}(\mathrm{ppm}) & 100 & 90 & 80 & 70 & 60 & 50 & 40 & 30 & 20 & 10 & 0 & -10 & -20\end{array}$ 


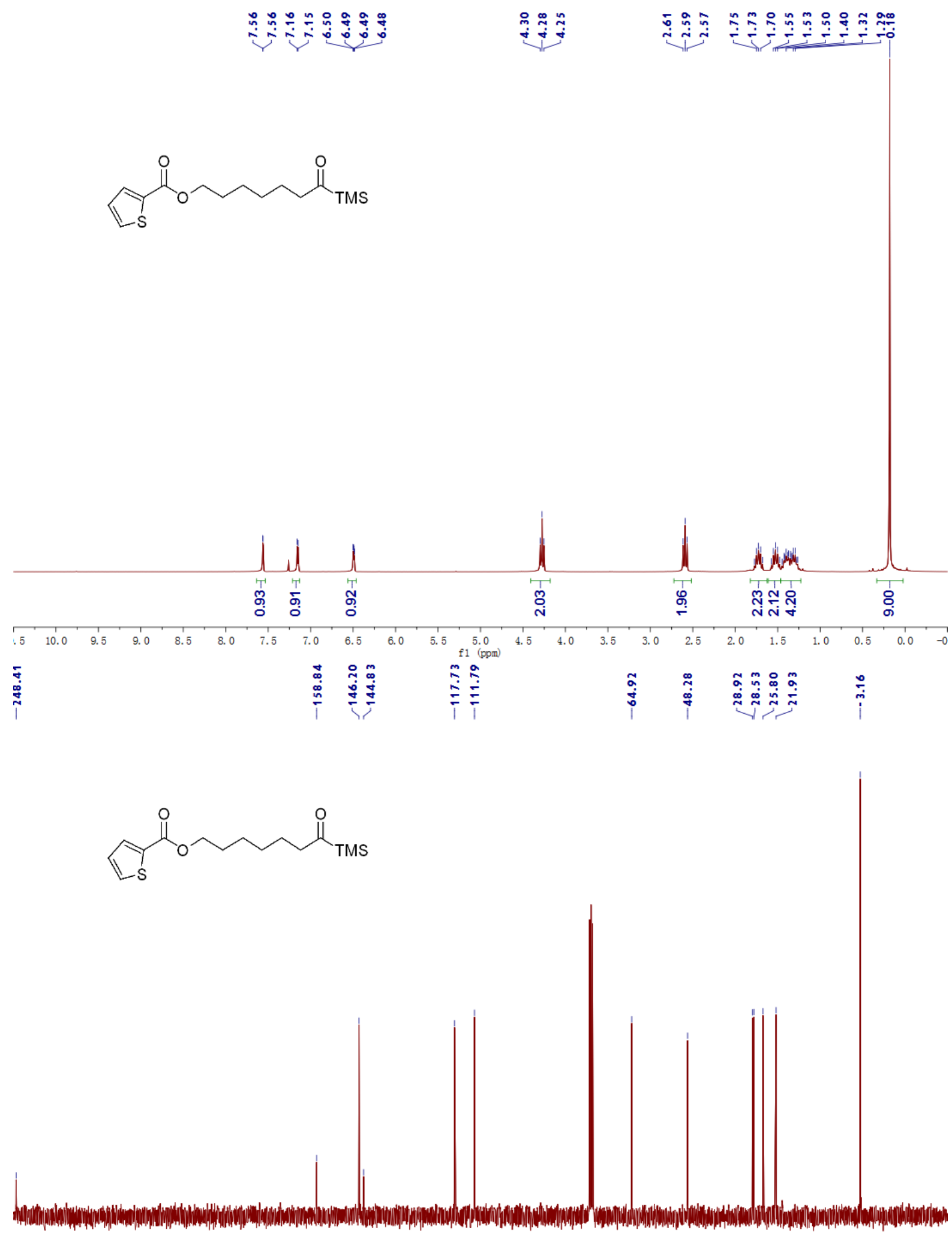

$\begin{array}{lllllllllllllllllllllllllll}240 & 230 & 220 & 210 & 200 & 190 & 180 & 170 & 160 & 150 & 140 & 130 & 120 & 110 & 100 & 90 & 80 & 70 & 60 & 50 & 40 & 30 & 20 & 10 & 0 & -10 & -20\end{array}$ 
<smiles>COC(CCc1ccccc1)C(Cc1ccccc1)O[Na]</smiles>
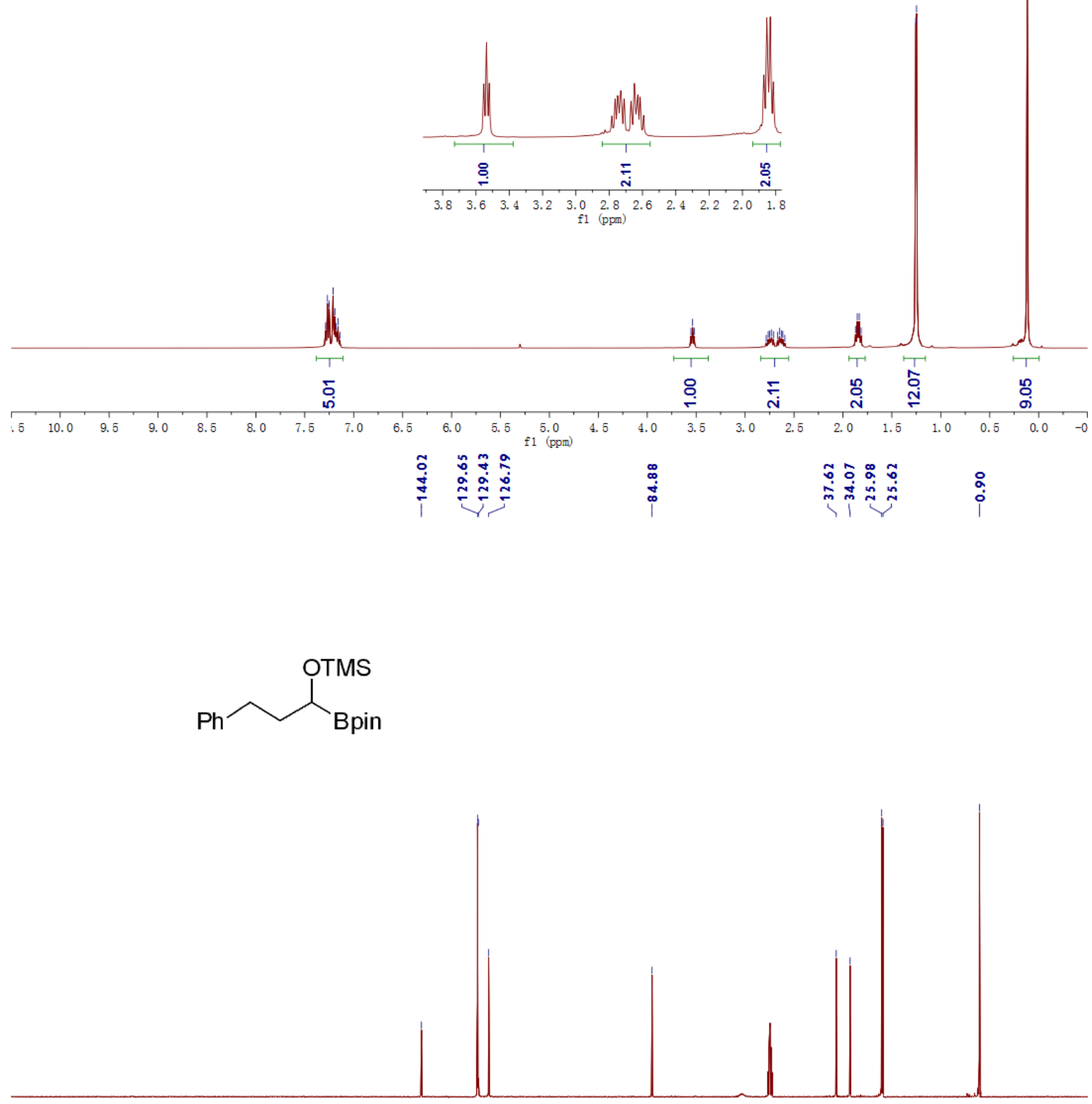

$\begin{array}{llllllllllllllllllllllllllllllllllll}240 & 230 & 220 & 210 & 200 & 190 & 180 & 170 & 160 & 150 & 140 & 130 & 120 & 110 & 100 & 90 & 80 & 70 & 60 & 50 & 40 & 30 & 20 & 10 & 0 & -10 & -20\end{array}$ 

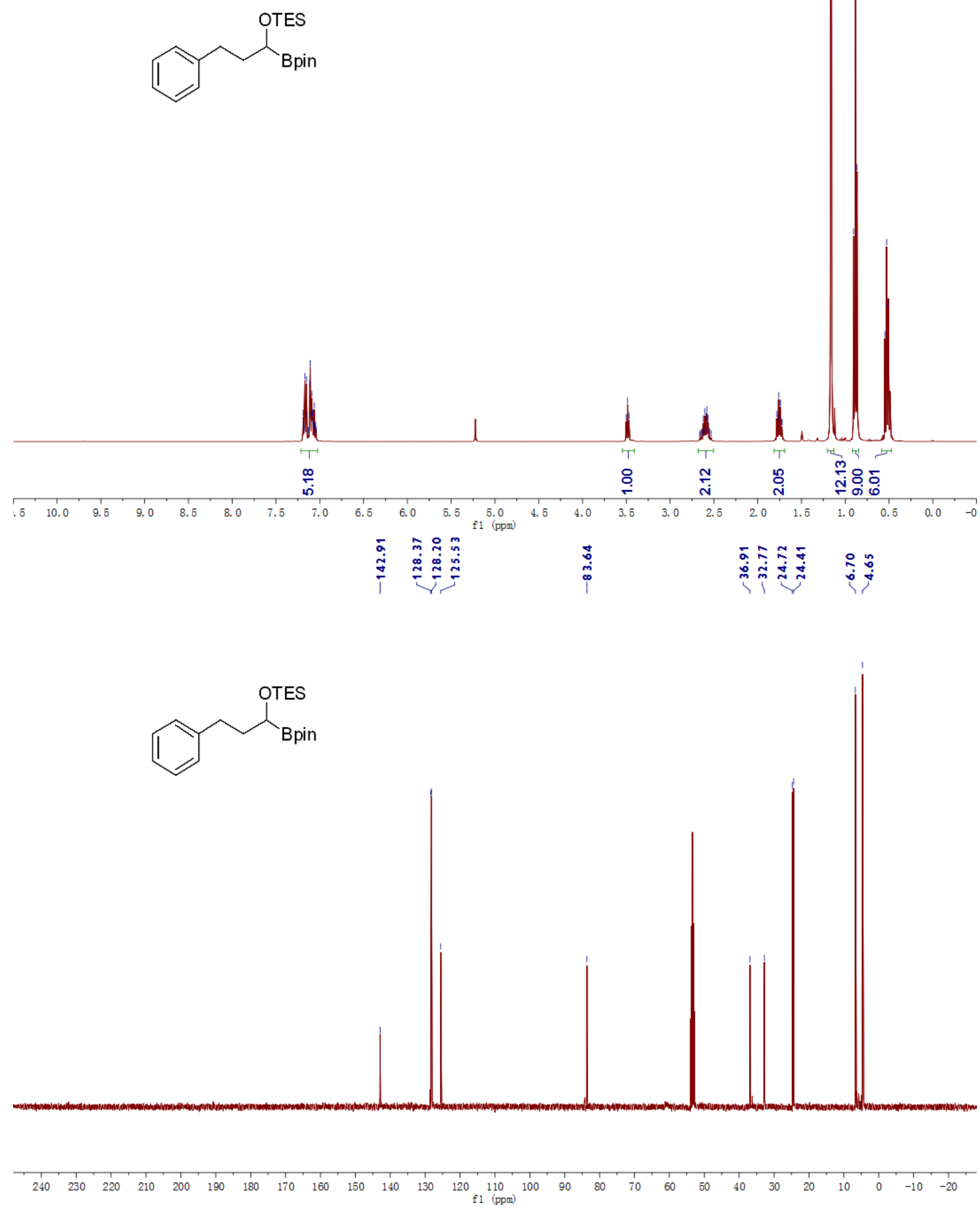


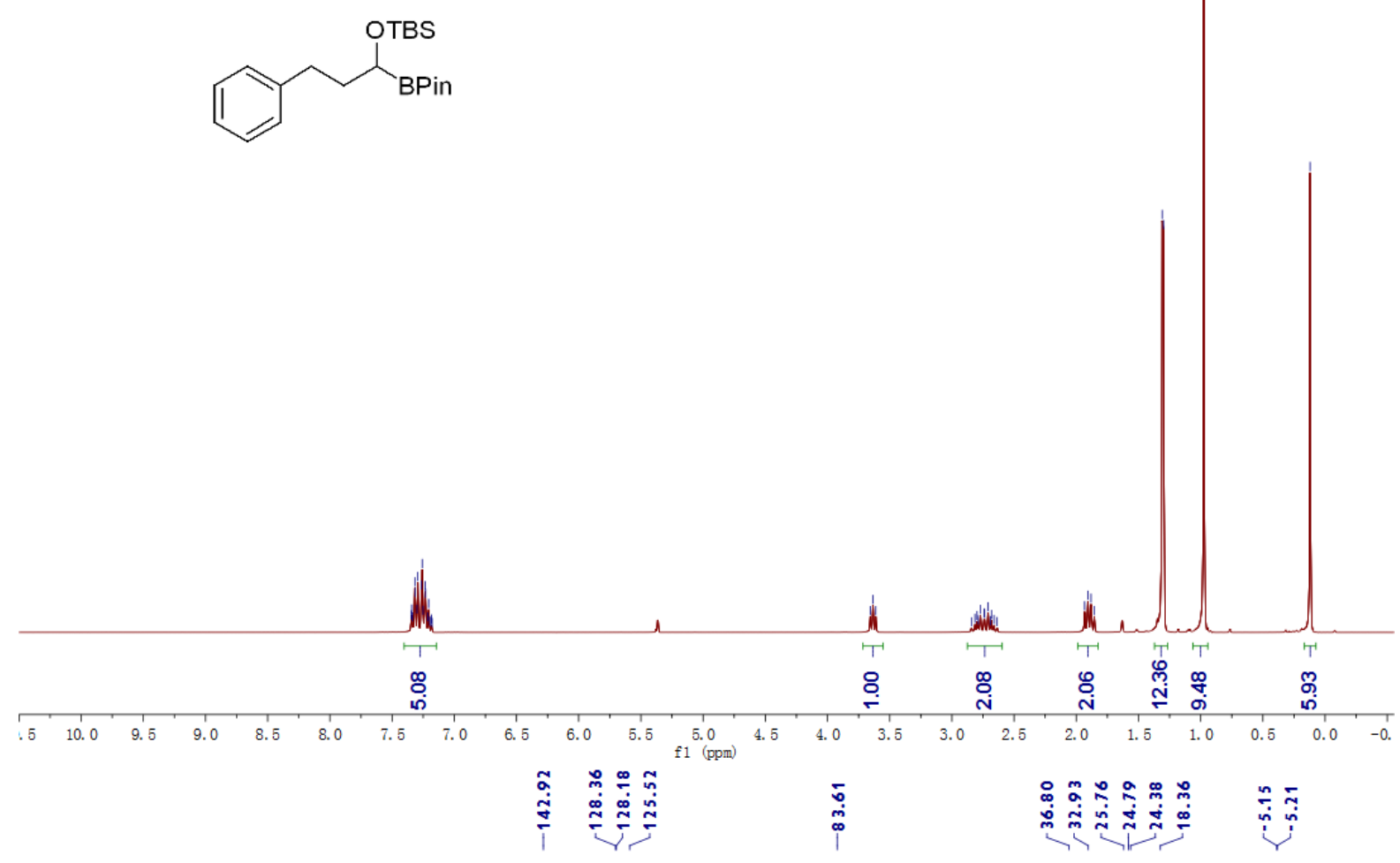

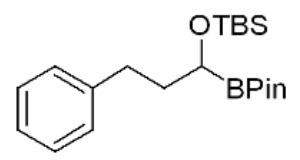

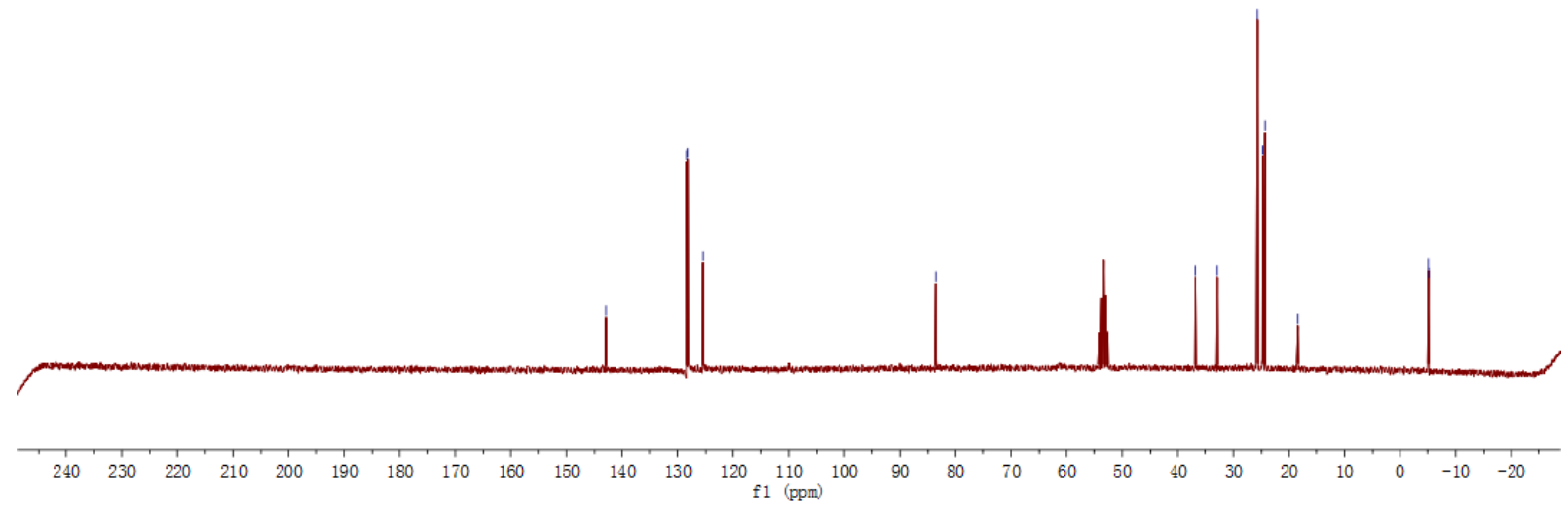


<smiles>CCCCC(Cc1ccccc1)OCc1ccccc1</smiles>
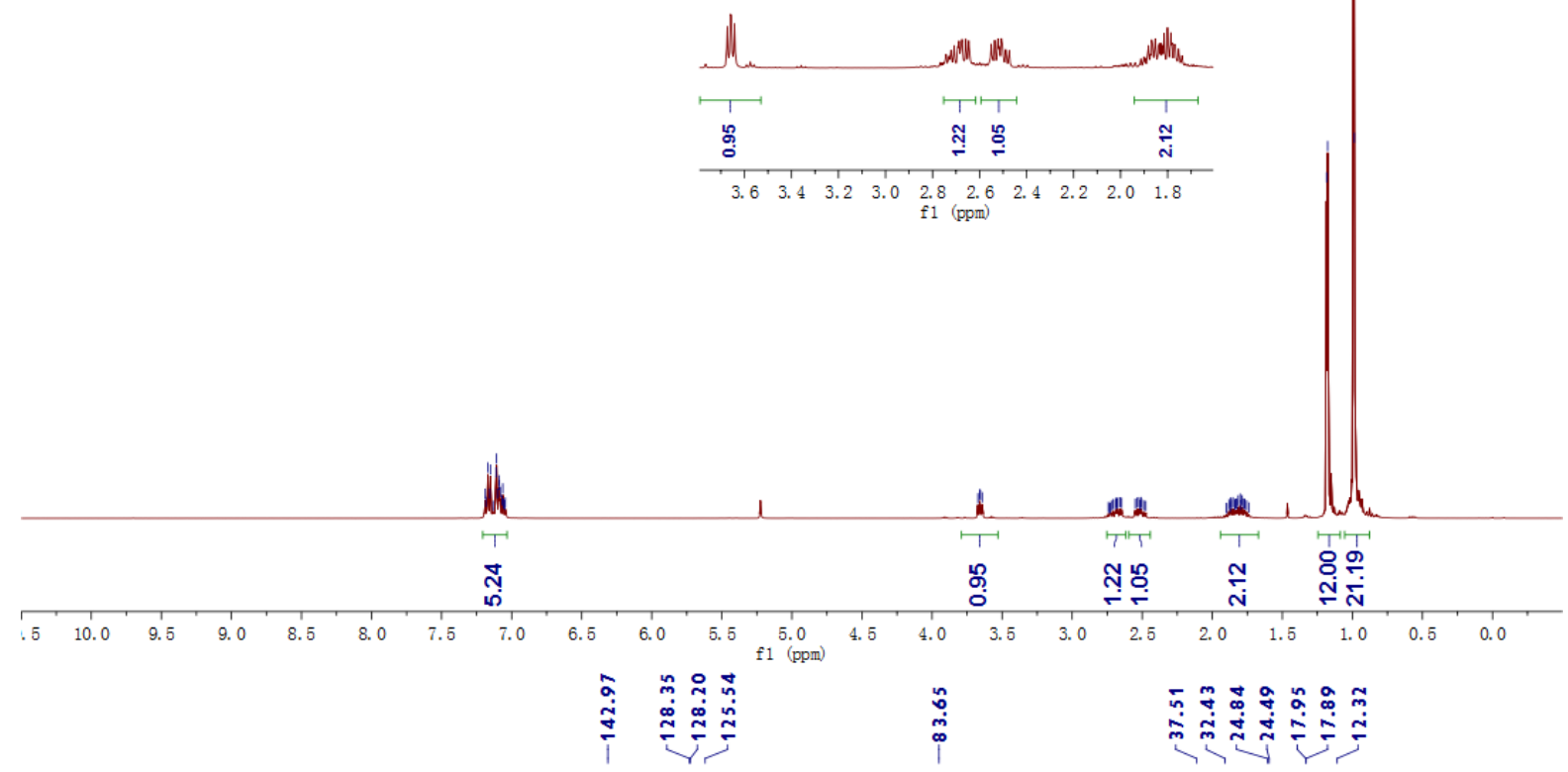<smiles>COC(CCc1ccccc1)Cc1ccccc1</smiles>

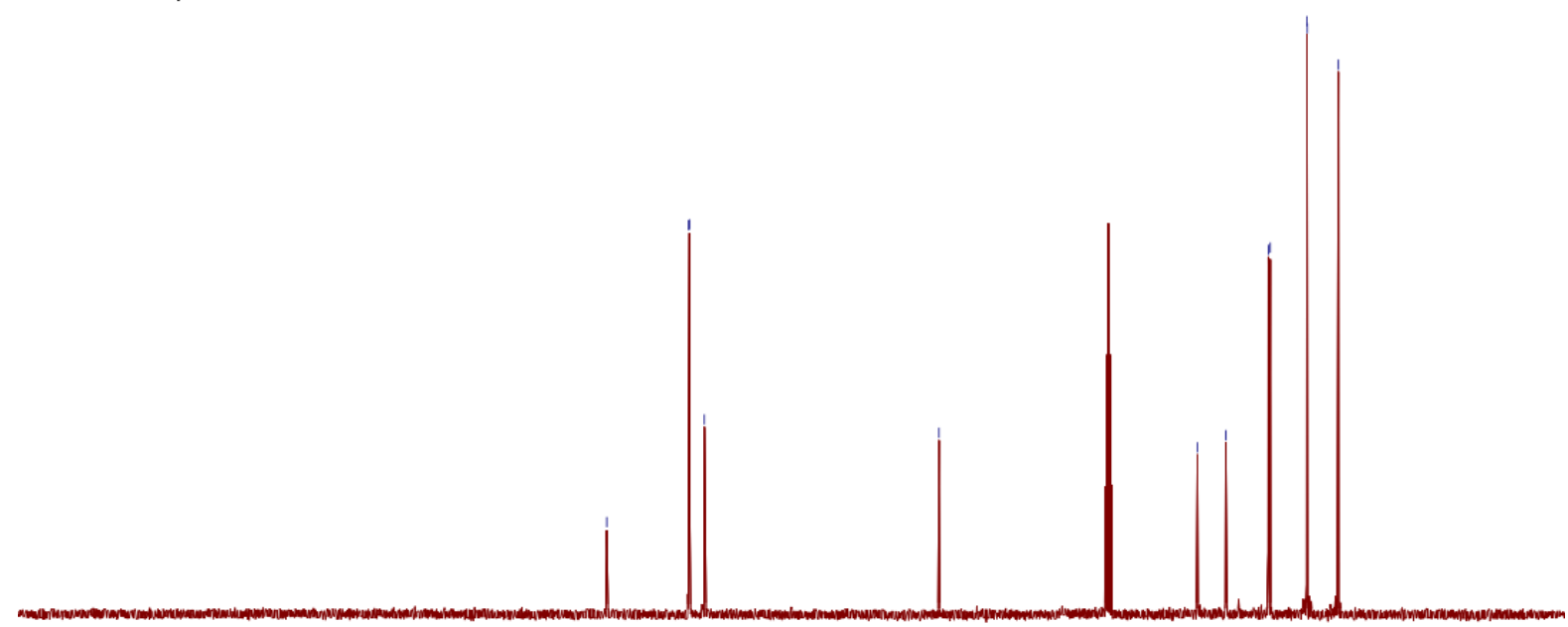

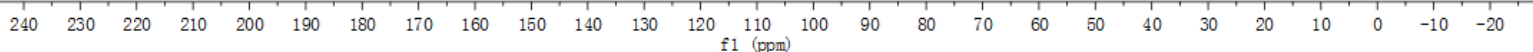



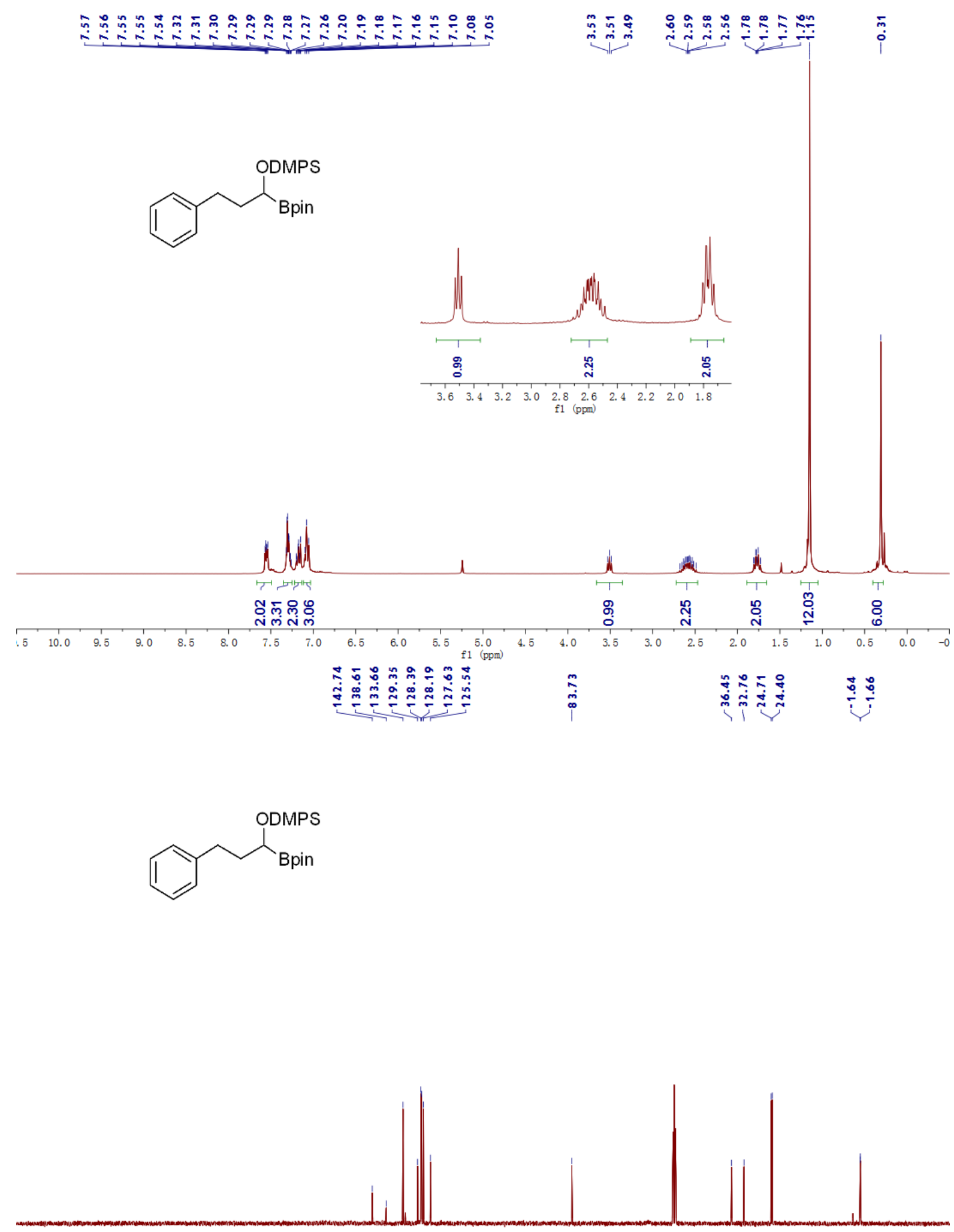

$\begin{array}{llllllllllllllllllllllllllllllllllll}240 & 230 & 220 & 210 & 200 & 190 & 180 & 170 & 160 & 150 & 140 & 130 & 120 & 110 & 100 & 90 & 80 & 70 & 60 & 50 & 40 & 30 & 20 & 10 & 0 & -10 & -20\end{array}$ 

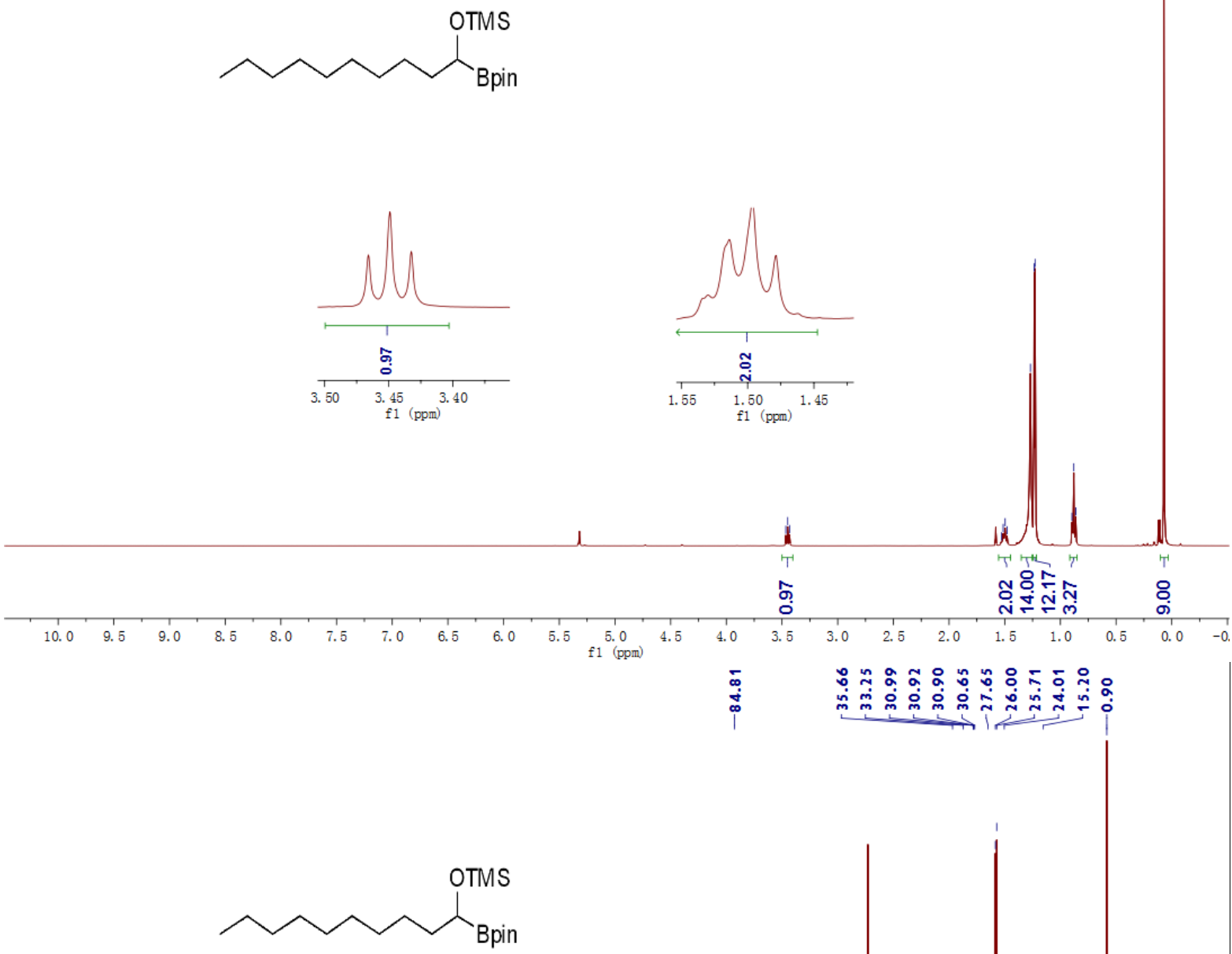

更 


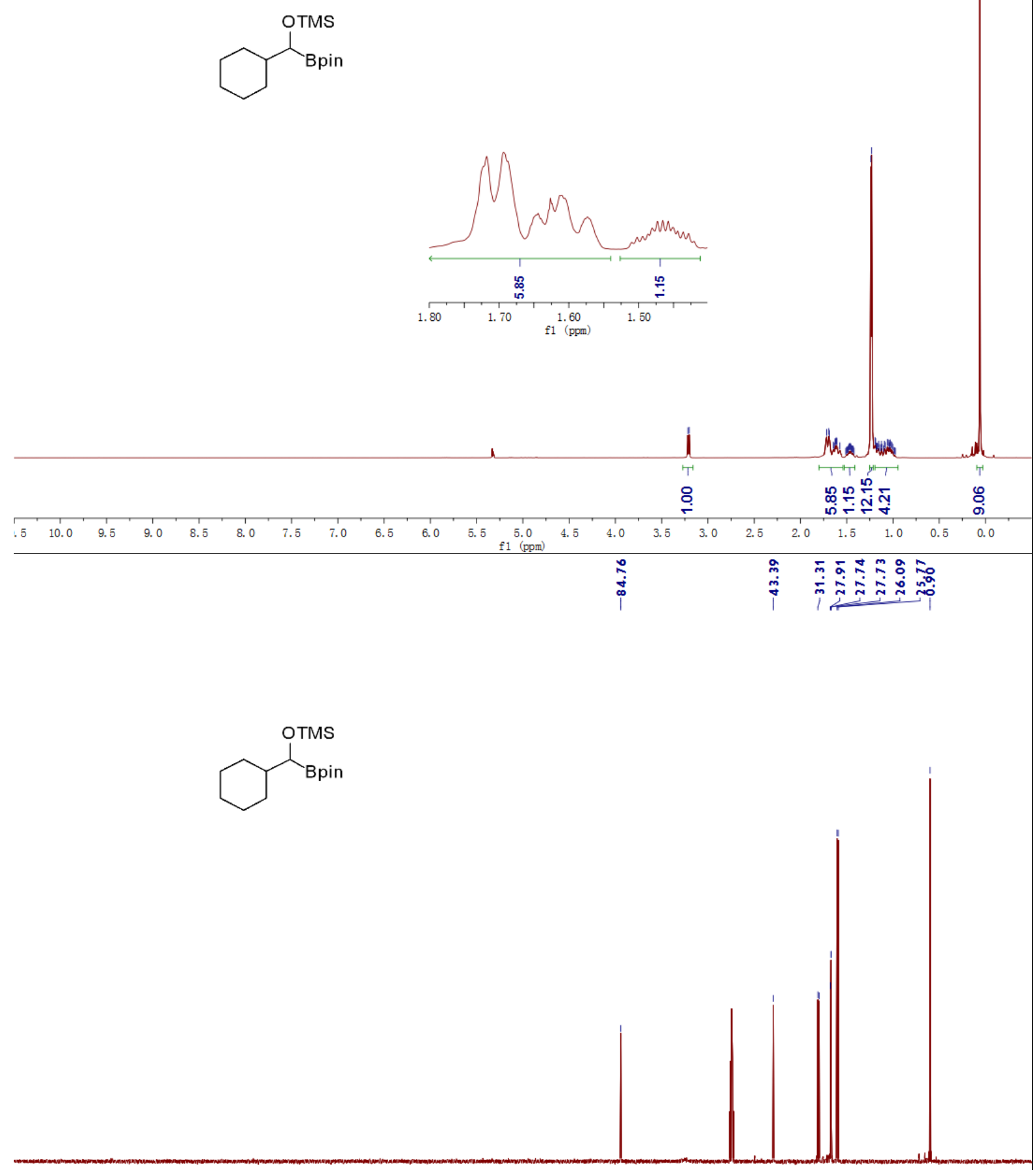

$\begin{array}{lllllllllllllllllllllllllll}240 & 230 & 220 & 210 & 200 & 190 & 180 & 170 & 160 & 150 & 140 & 130 & 120 & 110 & 100 & 90 & 80 & 70 & 60 & 50 & 40 & 30 & 20 & 10 & 0 & -10 & -20\end{array}$ 

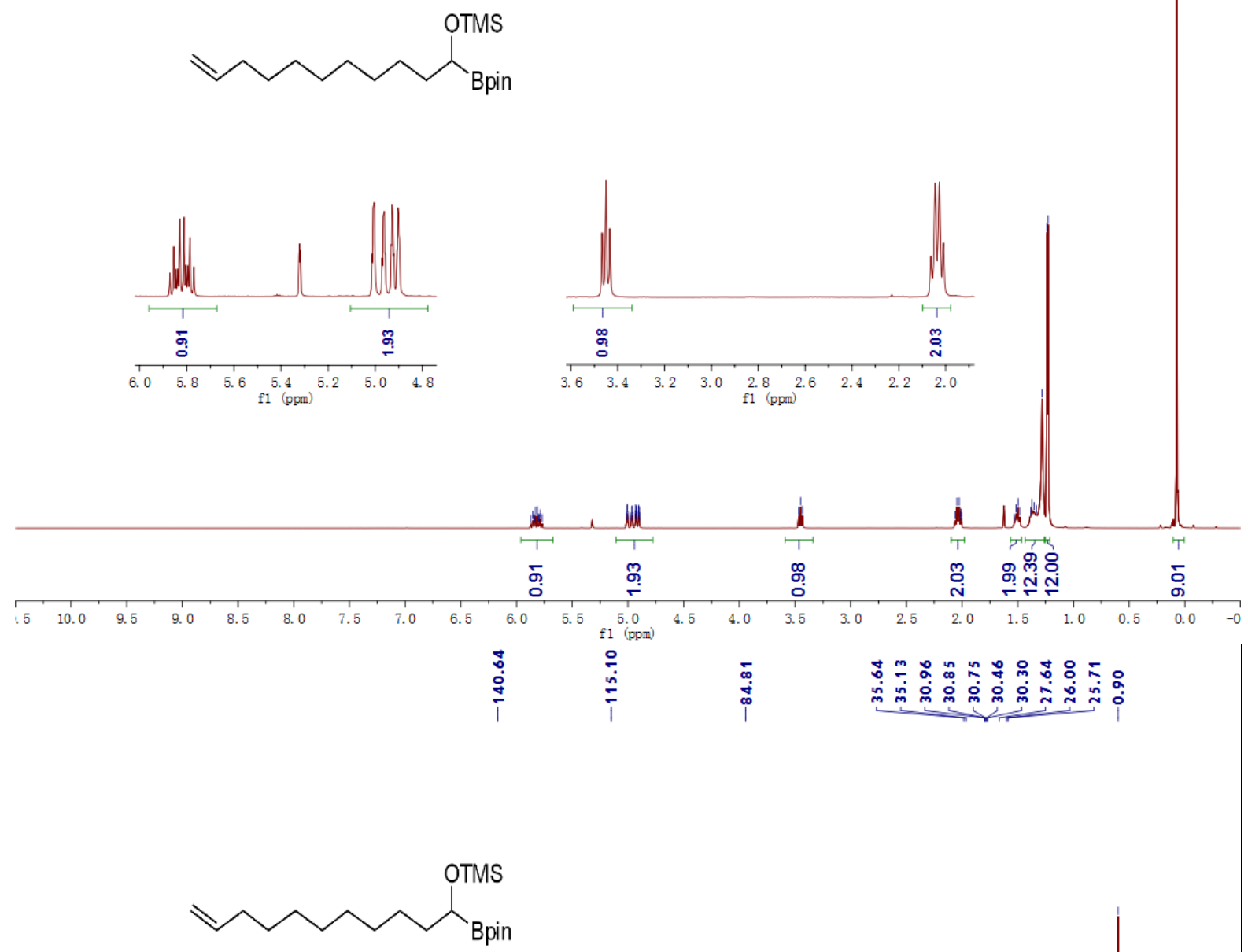

$\begin{array}{lllllllllllllllllllllllllllllll}240 & 230 & 220 & 210 & 200 & 190 & 180 & 170 & 160 & 150 & 140 & 130 & 120 & 110 & 100 & 90 & 80 & 70 & 60 & 50 & 40 & 30 & 20 & 10 & 0 & -10 & -20\end{array}$ 
<smiles>[B]C(CCCCCl)OC</smiles><smiles>[B]C(CCCCCl)OC</smiles>

$\begin{array}{llllllllllllllllllllllllllllllll}50 & 240 & 230 & 220 & 210 & 200 & 190 & 180 & 170 & 160 & 150 & 140 & 130 & 120 & 110 & 100 & 90 & 80 & 70 & 60 & 50 & 40 & 30 & 20 & 10 & 0 & -10 & -20\end{array}$ 


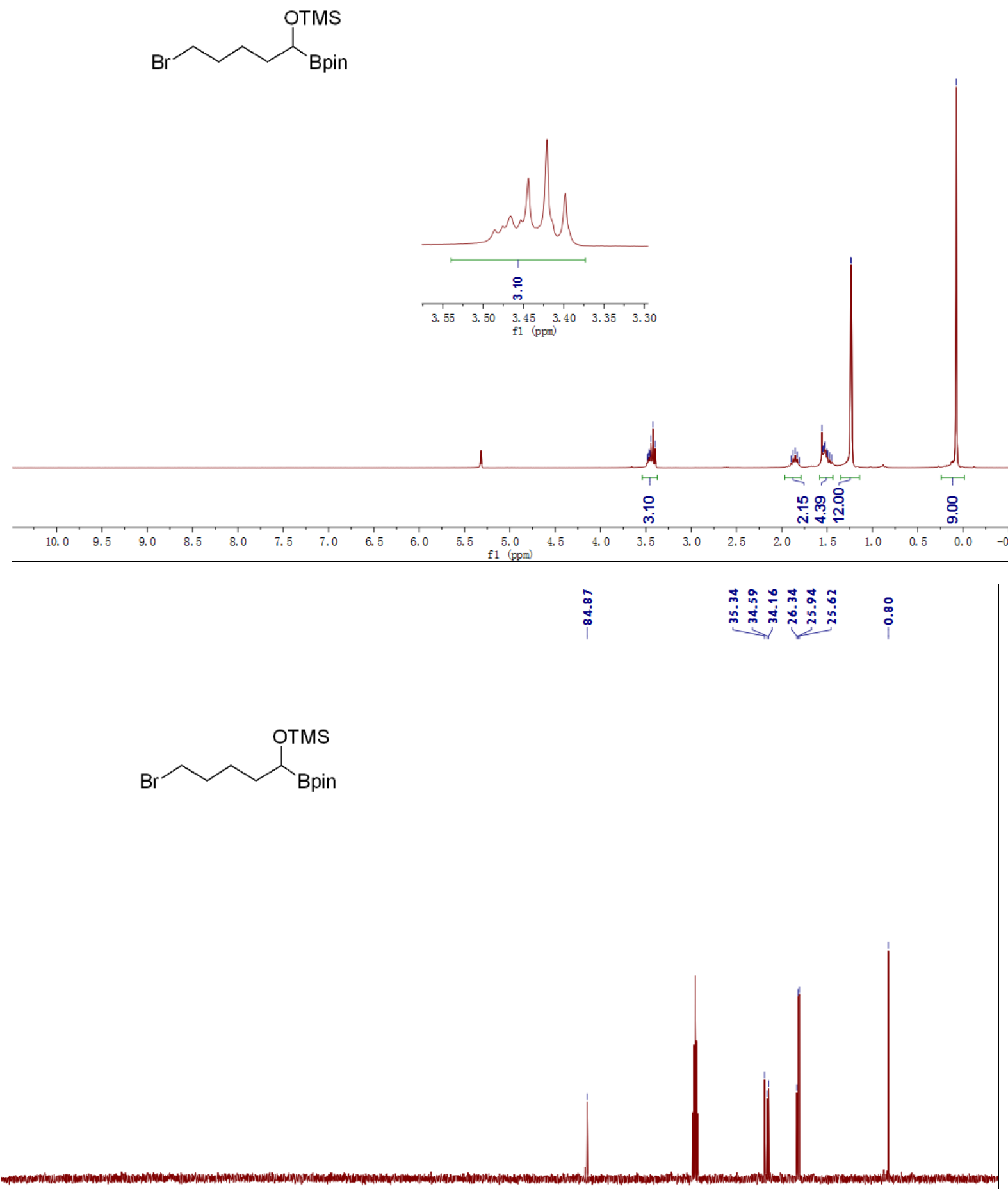



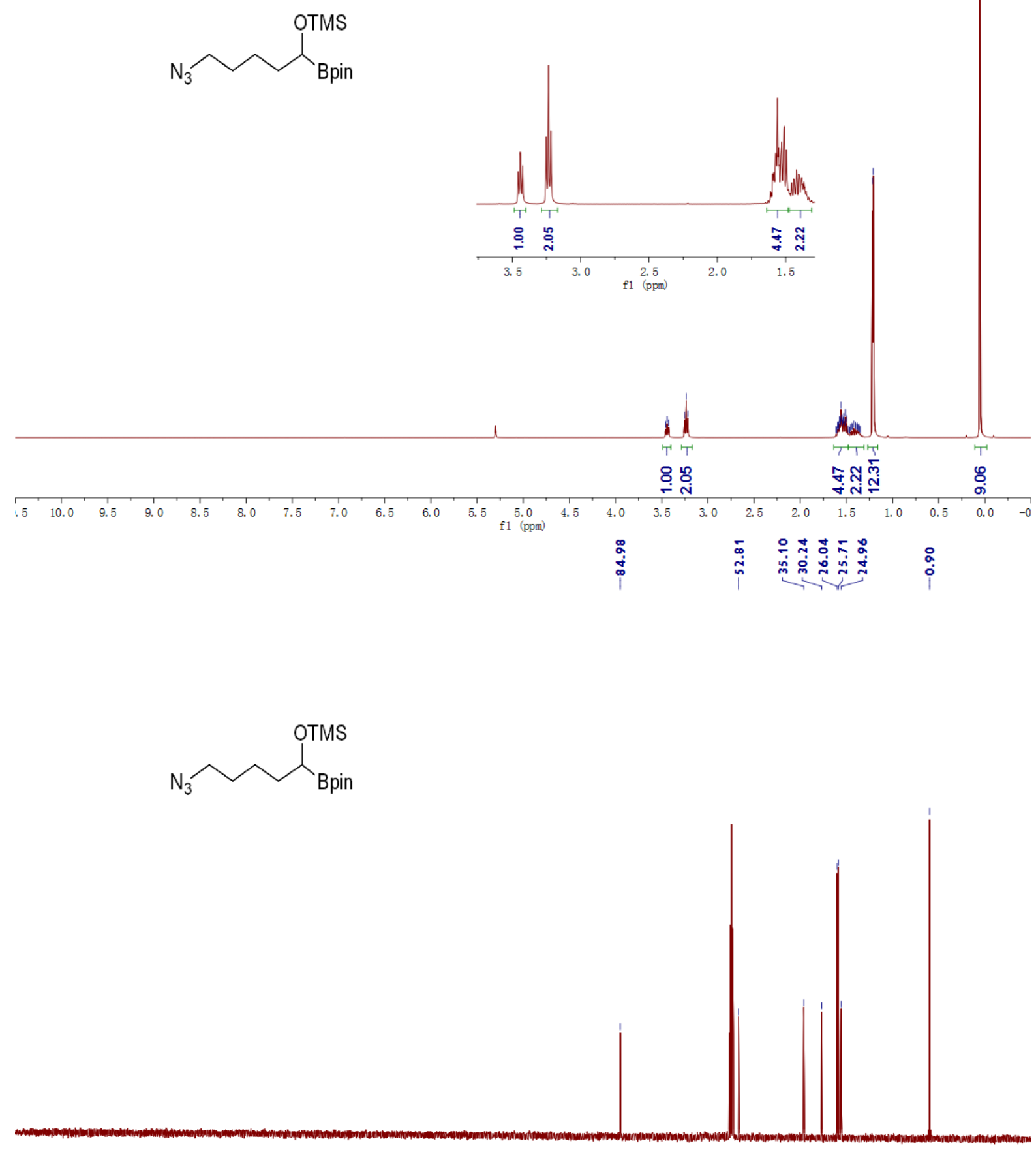

$\begin{array}{lllllllllllllllllllllllllllllll}240 & 230 & 220 & 210 & 200 & 190 & 180 & 170 & 160 & 150 & 140 & 130 & 120 & 110 & 100 & 90 & 80 & 70 & 60 & 50 & 40 & 30 & 20 & 10 & 0 & -10 & -20\end{array}$ 

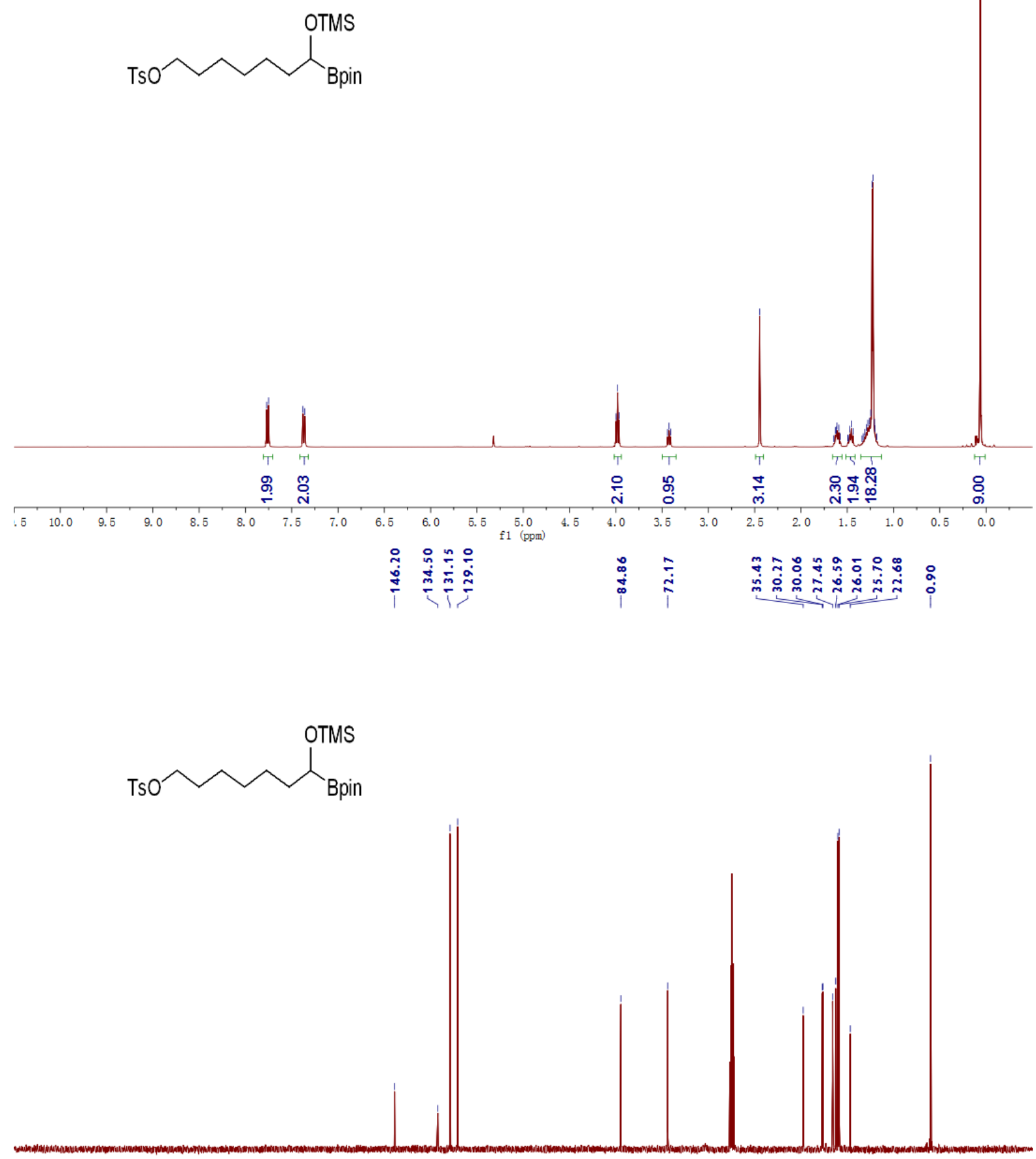

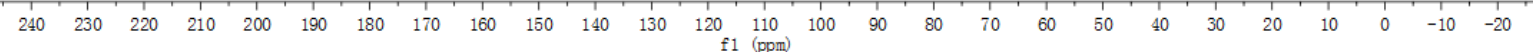


<smiles>COC(CCCCN1C(=O)c2ccccc2C1=O)Cc1ccccc1</smiles>
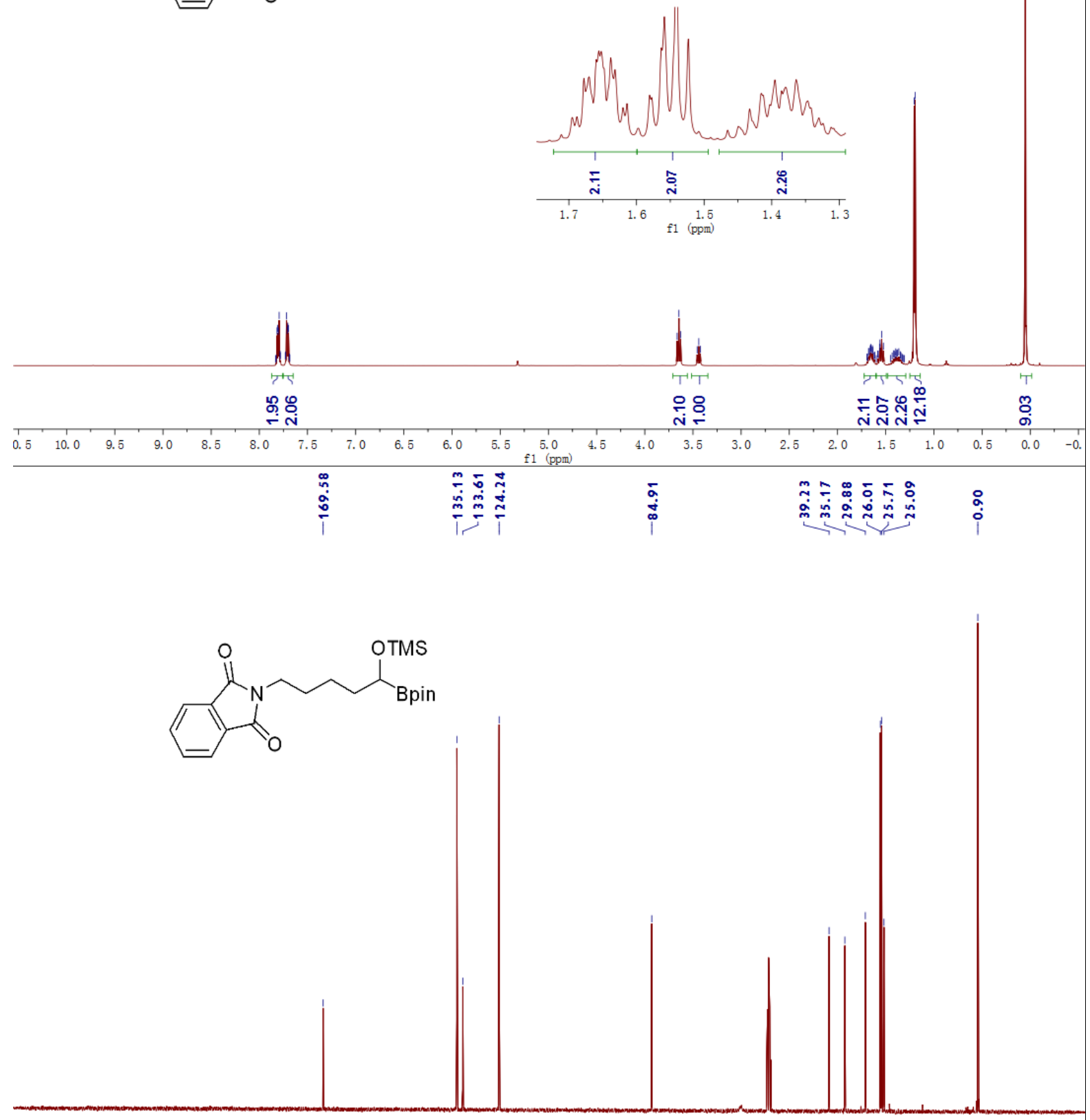

$\begin{array}{llllllllllllllllllllllllllllllll}240 & 230 & 220 & 210 & 200 & 190 & 180 & 170 & 160 & 150 & 140 & 130 & 120 & 110 & 100 & 90 & 80 & 70 & 60 & 50 & 40 & 30 & 20 & 10 & 0 & -10 & -20\end{array}$ 

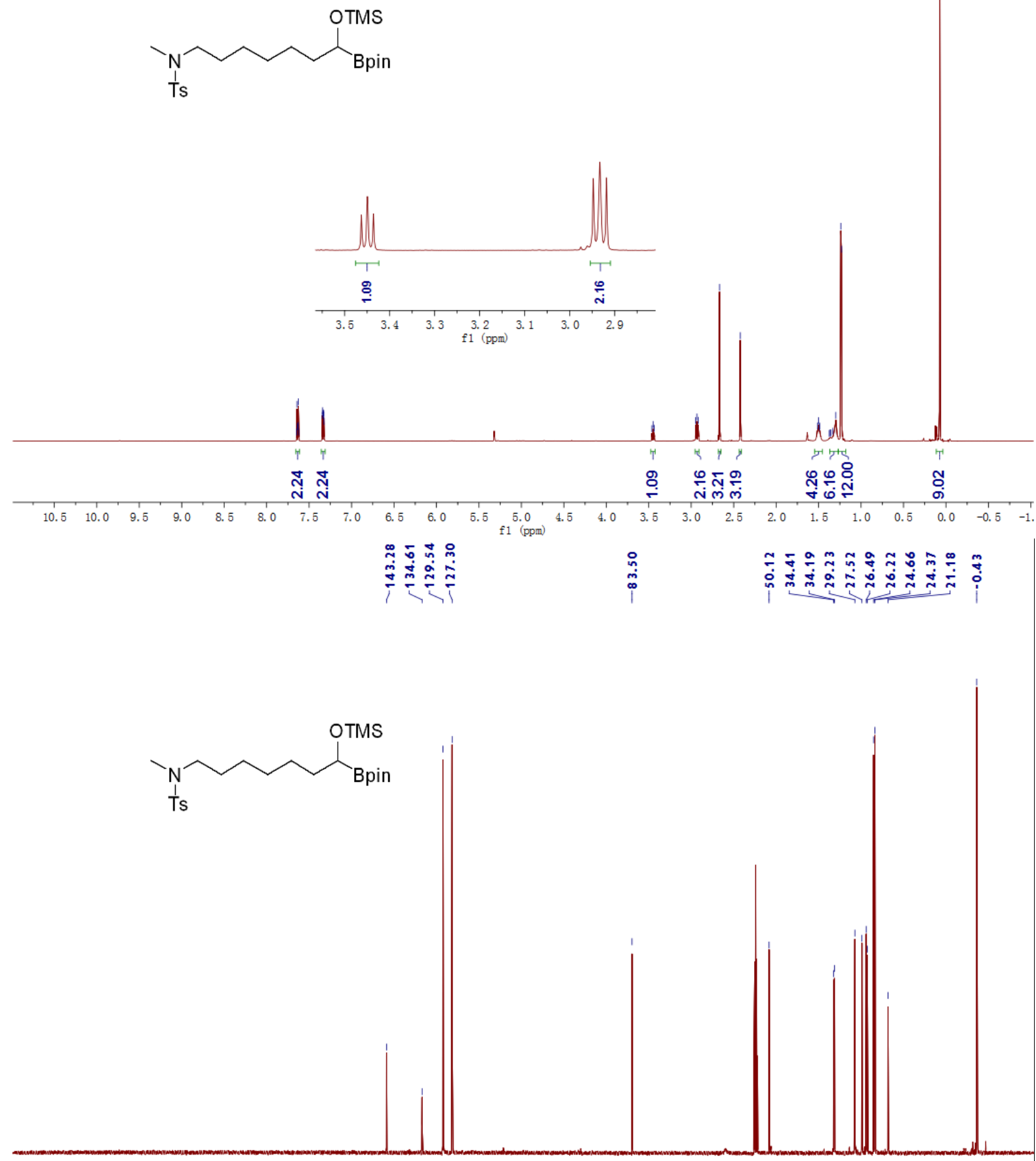

$\begin{array}{lllllllllllllllllllllllllllll}230 & 220 & 210 & 200 & 190 & 180 & 170 & 160 & 150 & 140 & 130 & 120 & 110 & 100 & 90 & 80 & 70 & 60 & 50 & 40 & 30 & 20 & 10 & 0 & -10\end{array}$ 

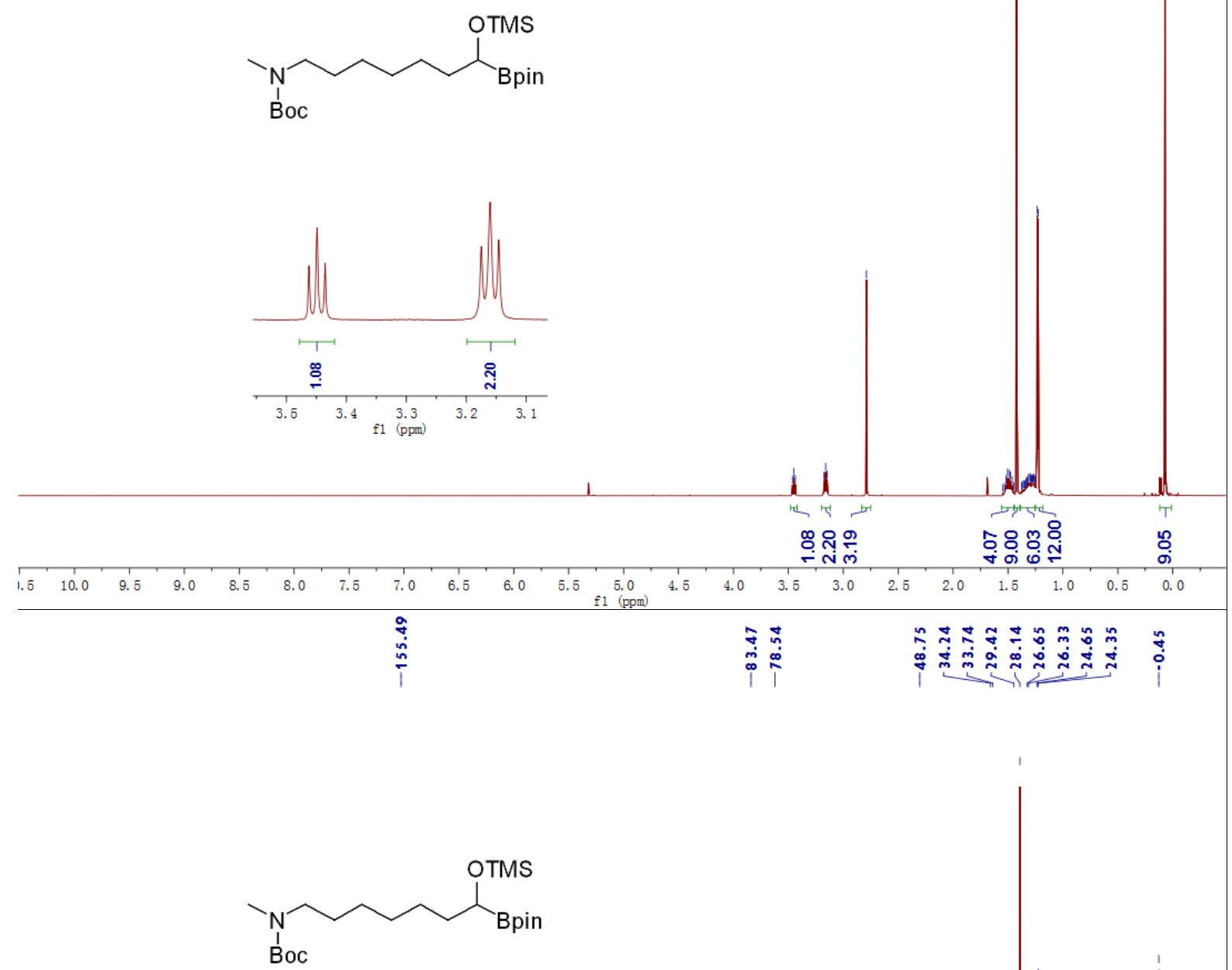

\begin{tabular}{llllllllllllllllllllllllllll}
\hline 230 & 220 & 210 & 200 & 190 & 180 & 170 & 160 & 150 & 140 & 130 & 120 & 110 & 100 & 90 & 80 & 70 & 60 & 50 & 40 & 30 & 20 & 10 & 0 & -10
\end{tabular} 
<smiles>COC([B]O)CC(C)(C)C(=O)O</smiles>

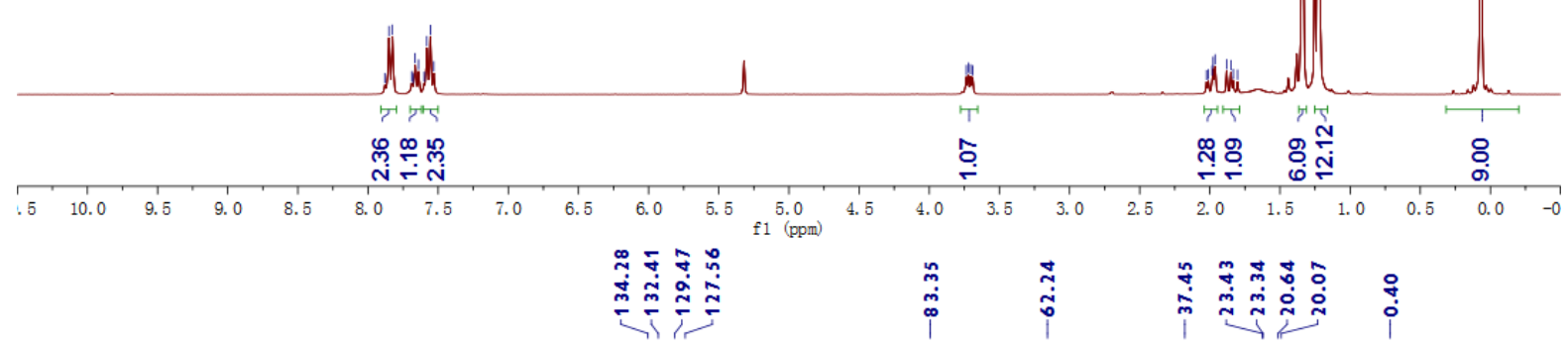

$\underbrace{\text { OTMS }}_{\text {Bhin }}$

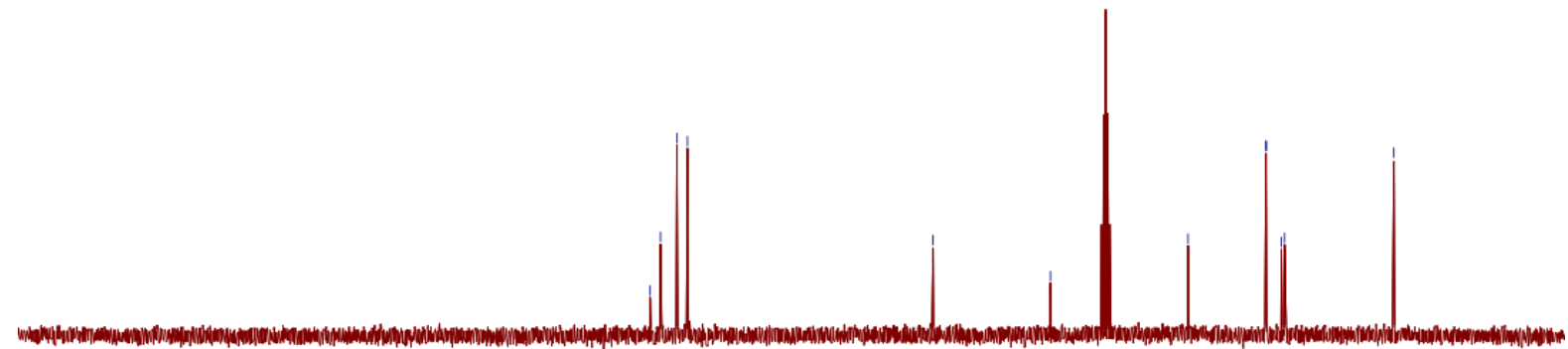

$\begin{array}{lllllllllllllllllllllllllllll}240 & 230 & 220 & 210 & 200 & 190 & 180 & 170 & 160 & 150 & 140 & 130 & 120 & 110 & 100 & 90 & 80 & 70 & 60 & 50 & 40 & 30 & 20 & 10 & 0 & -10 & -20 & -3\end{array}$ 


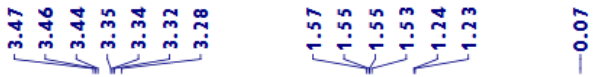

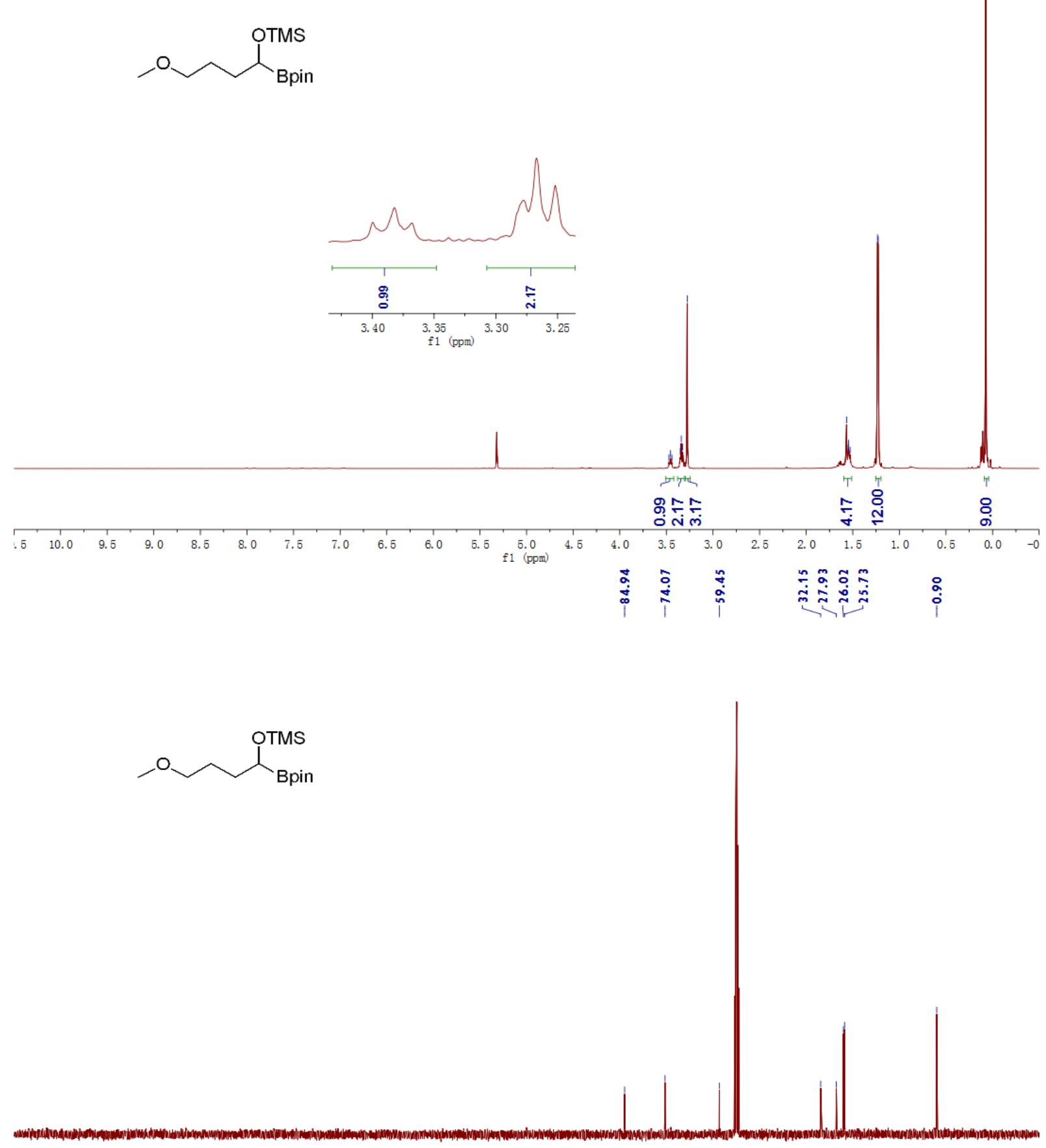

$\begin{array}{lllllllllllllllllllllllllllll}240 & 230 & 220 & 210 & 200 & 190 & 180 & 170 & 160 & 150 & 140 & 130 & 120 & 110 & 100 & 90 & 80 & 70 & 60 & 50 & 40 & 30 & 20 & 10 & 0 & -10 & -20\end{array}$ 

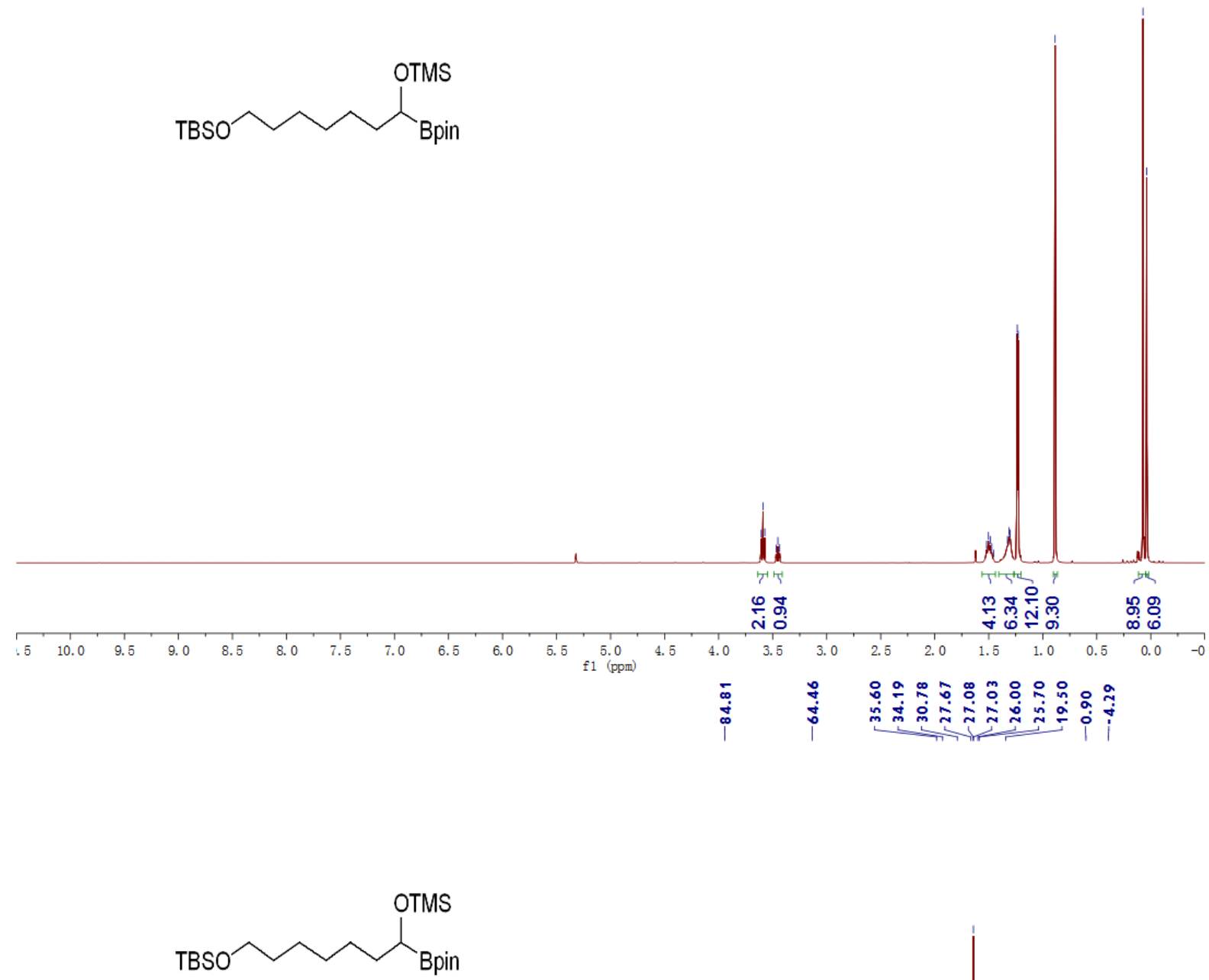

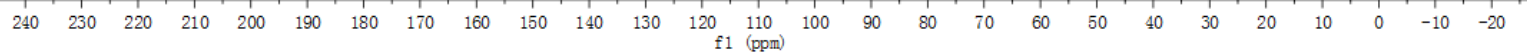




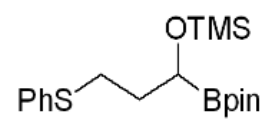
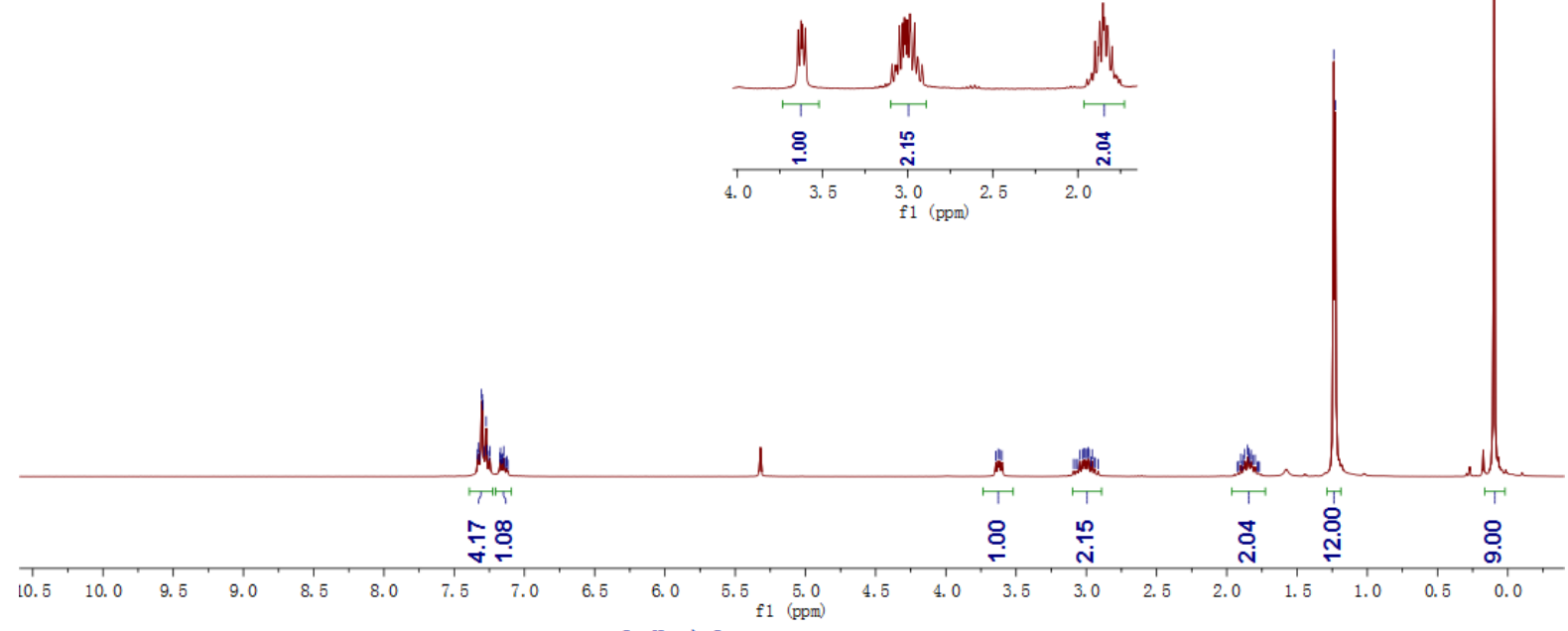

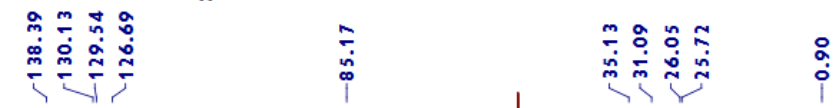

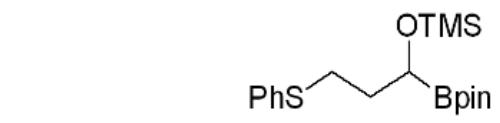

$\begin{array}{lllllllllllllllllllllllllllll}240 & 230 & 220 & 210 & 200 & 190 & 180 & 170 & 160 & 150 & 140 & 130 & 120 & 110 & 100 & 90 & 80 & 70 & 60 & 50 & 40 & 30 & 20 & 10 & 0 & -10 & -20\end{array}$ 
<smiles>COC(CCCCCCOC(=O)c1ccccc1)C(OC)c1ccccc1</smiles>
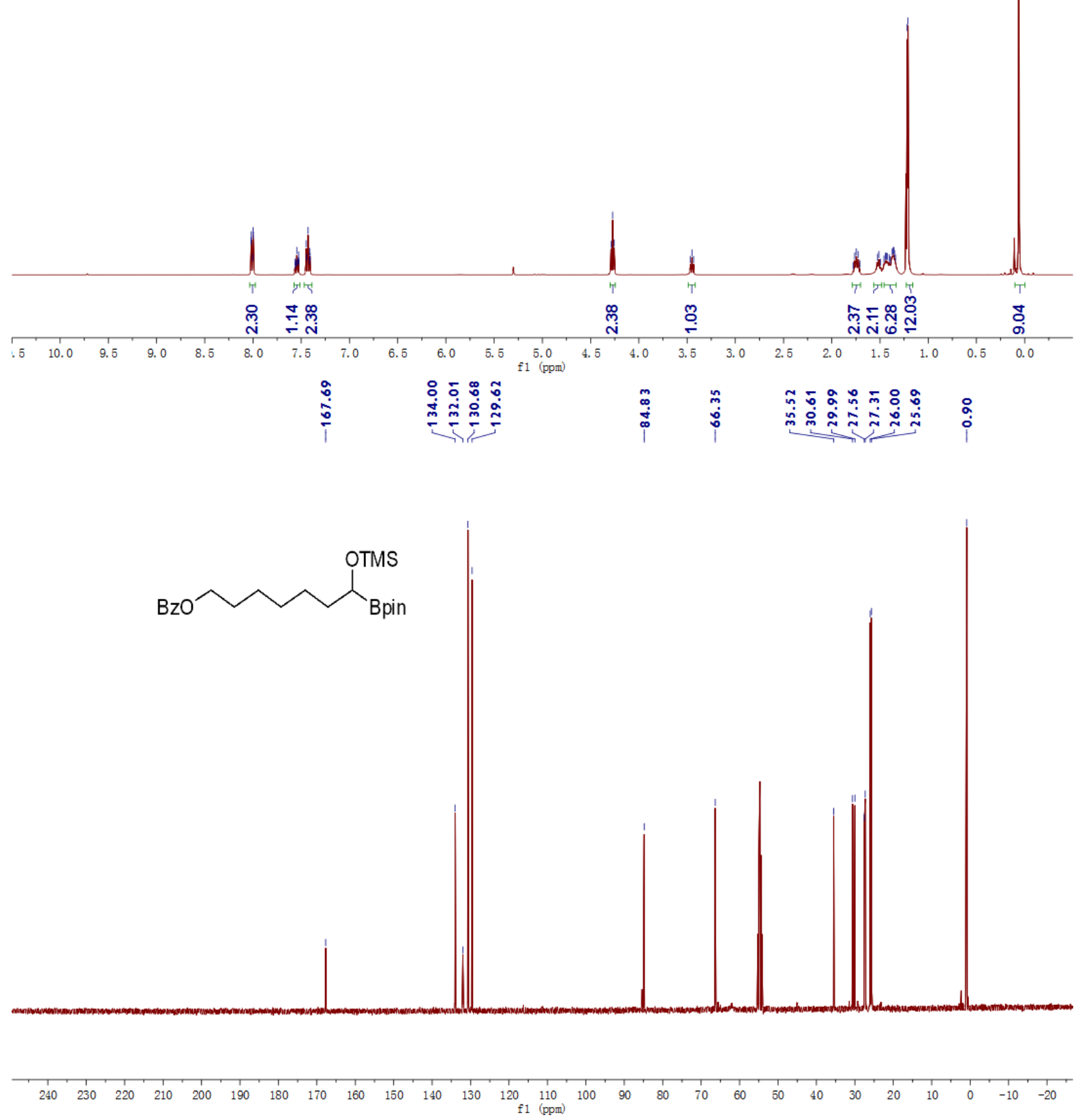

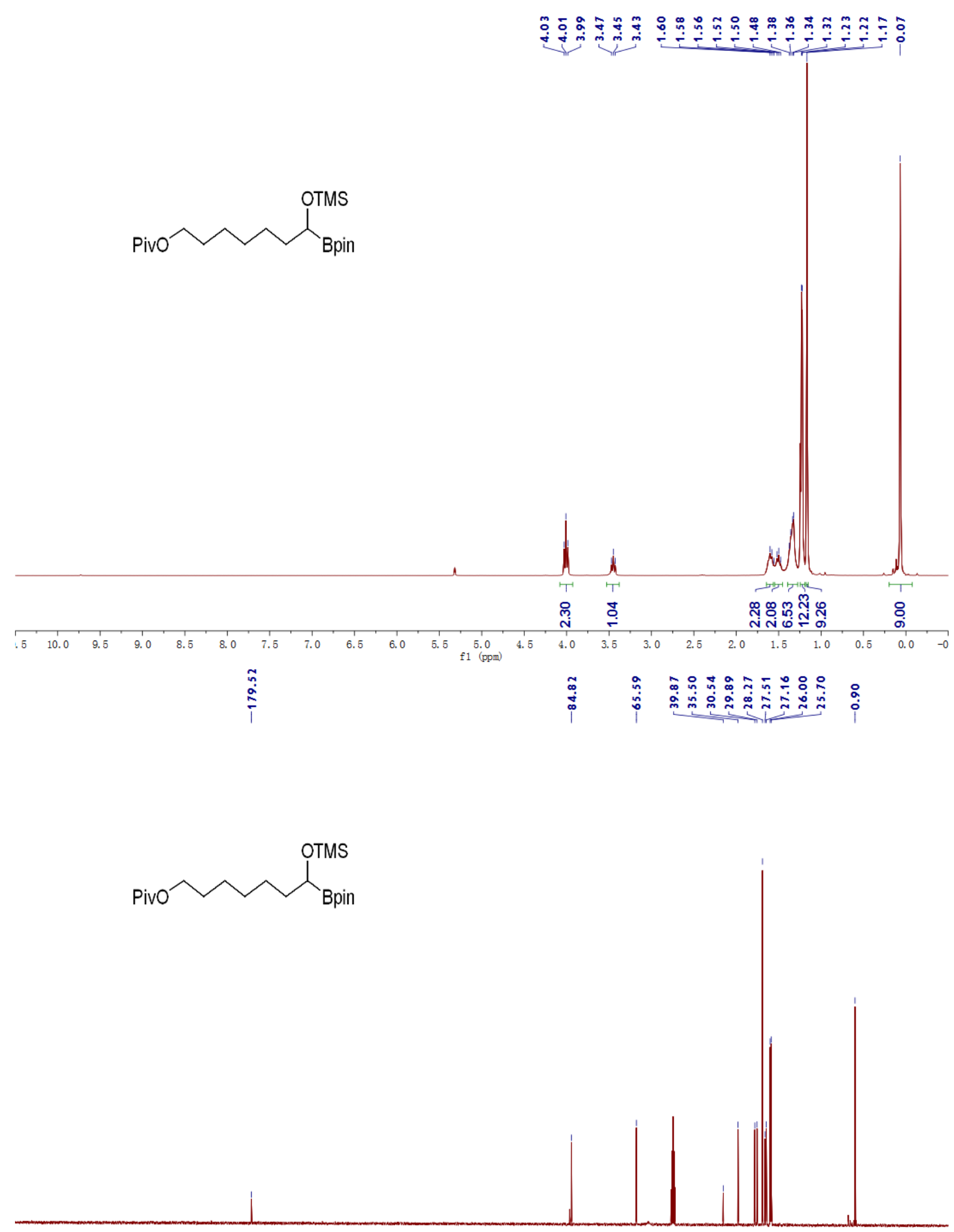

$\begin{array}{llllllllllllllllllllllllllllllll}240 & 230 & 220 & 210 & 200 & 190 & 180 & 170 & 160 & 150 & 140 & 130 & 120 & 110 & 100 & 90 & 80 & 70 & 60 & 50 & 40 & 30 & 20 & 10 & 0 & -10 & -20\end{array}$ 
<smiles>COC(CCCCCCOC(=O)c1ccco1)Cc1ccccc1</smiles>

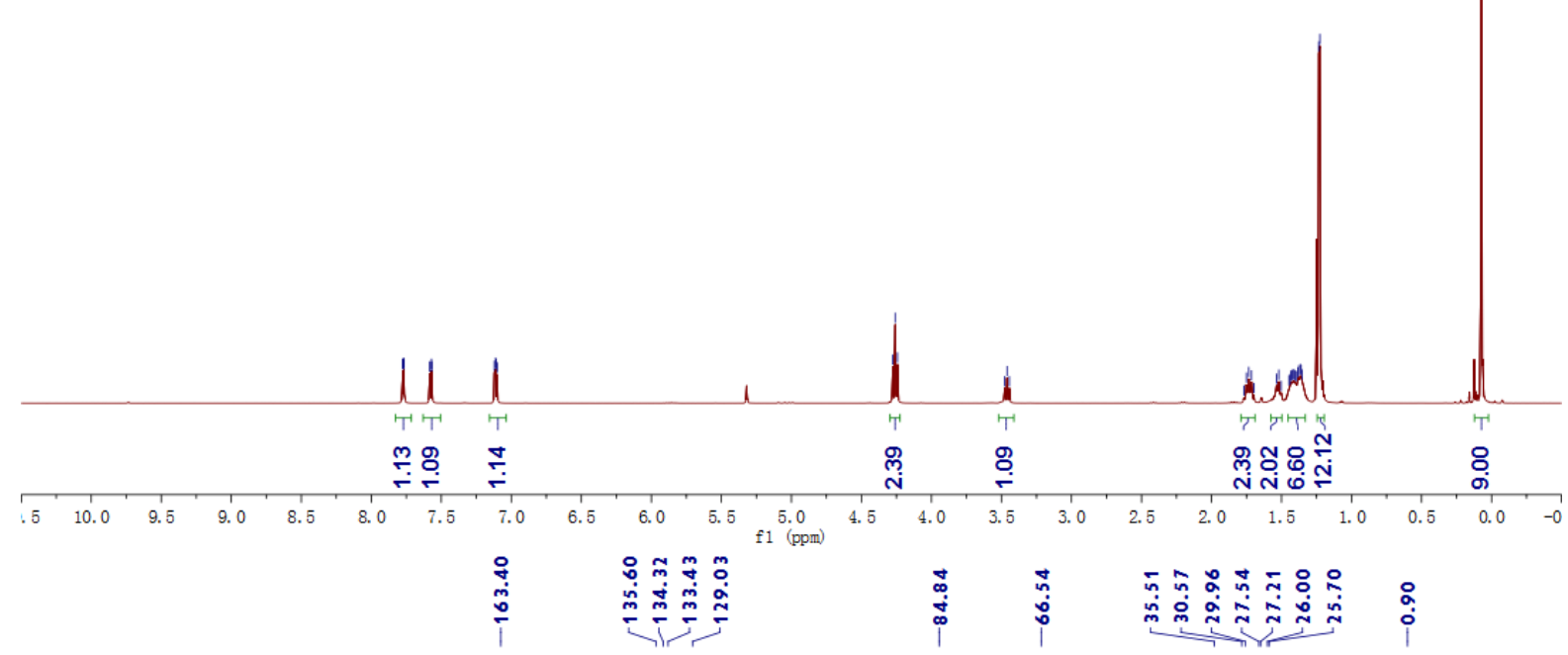<smiles>COC(CCCCCCOC(=O)c1ccco1)Cc1ccccc1</smiles>

$\begin{array}{lllllllllllllllllllllllllll}240 & 230 & 220 & 210 & 200 & 190 & 180 & 170 & 160 & 150 & 140 & 130 & 120 & \begin{array}{ll}110 \\ \text { (ppm) }\end{array} & 100 & 90 & 80 & 70 & 60 & 50 & 40 & 30 & 20 & 10 & 0 & -10 & -20\end{array}$ 
<smiles>COC(CCCCCCOC(=O)c1cccs1)Cc1ccccc1</smiles>

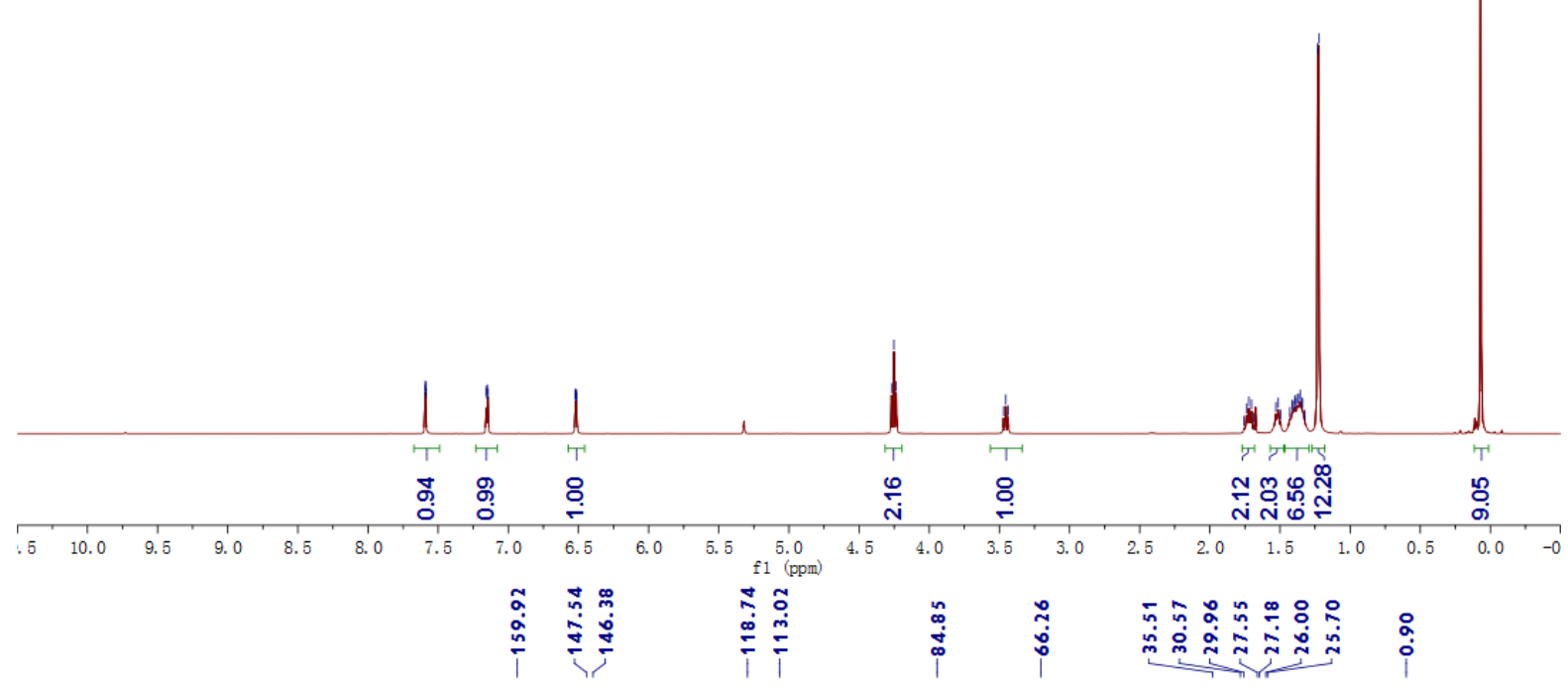<smiles>COC(CCCCCCOC(=O)c1cccs1)Cc1ccccc1</smiles>

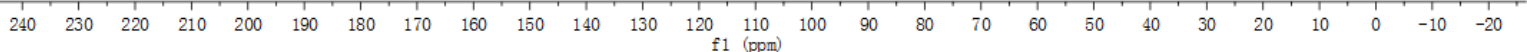


<smiles>COC(CCCCN1C(=O)c2ccccc2S1(=O)(O)O)C(Cc1ccccc1)Cc1ccccc1</smiles>
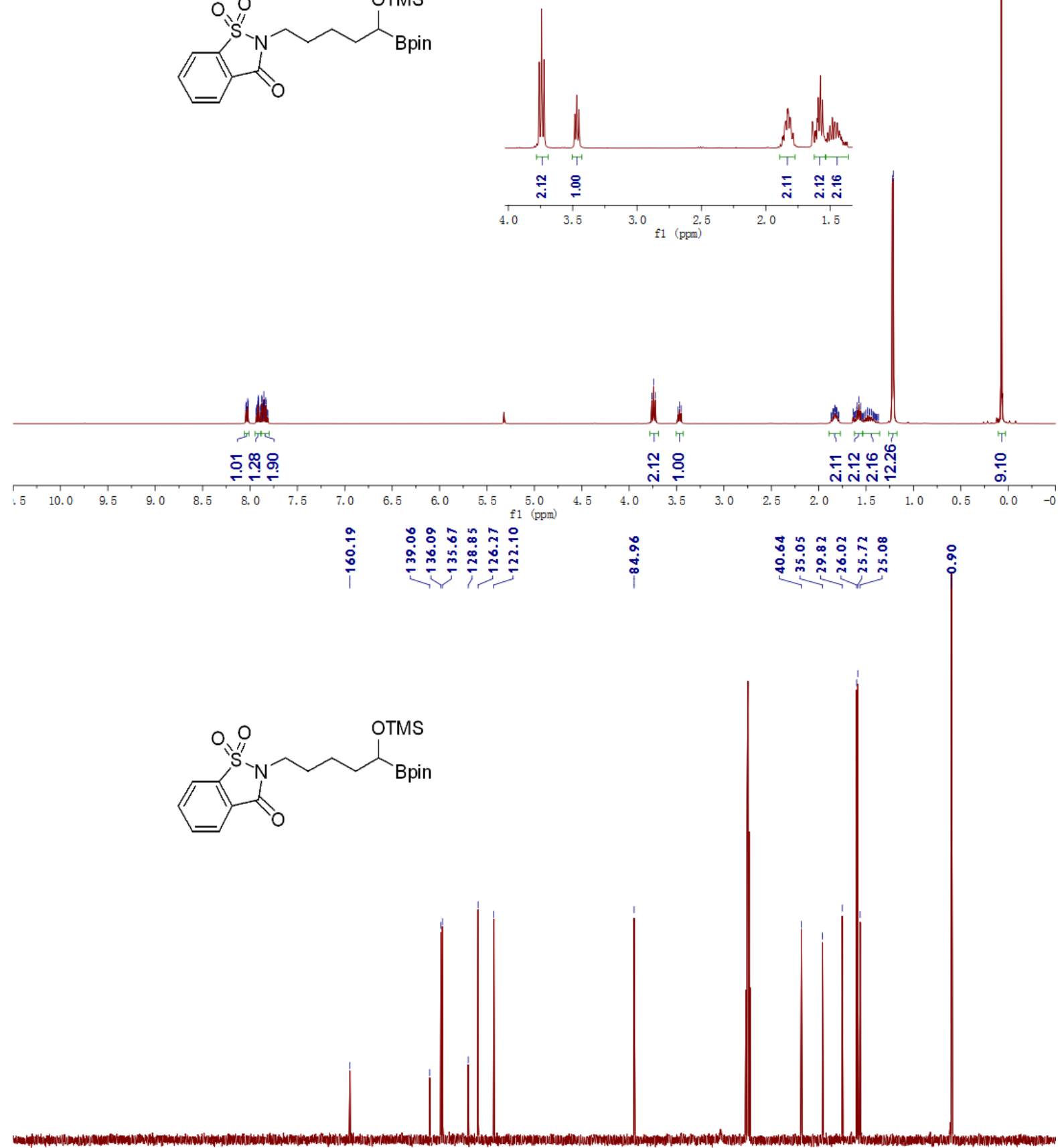

$\begin{array}{llllllllllllllllllllllllllllllllll}240 & 230 & 220 & 210 & 200 & 190 & 180 & 170 & 160 & 150 & 140 & 130 & 120 & 110 & 100 & 90 & 80 & 70 & 60 & 50 & 40 & 30 & 20 & 10 & 0 & -10 & -20\end{array}$ 

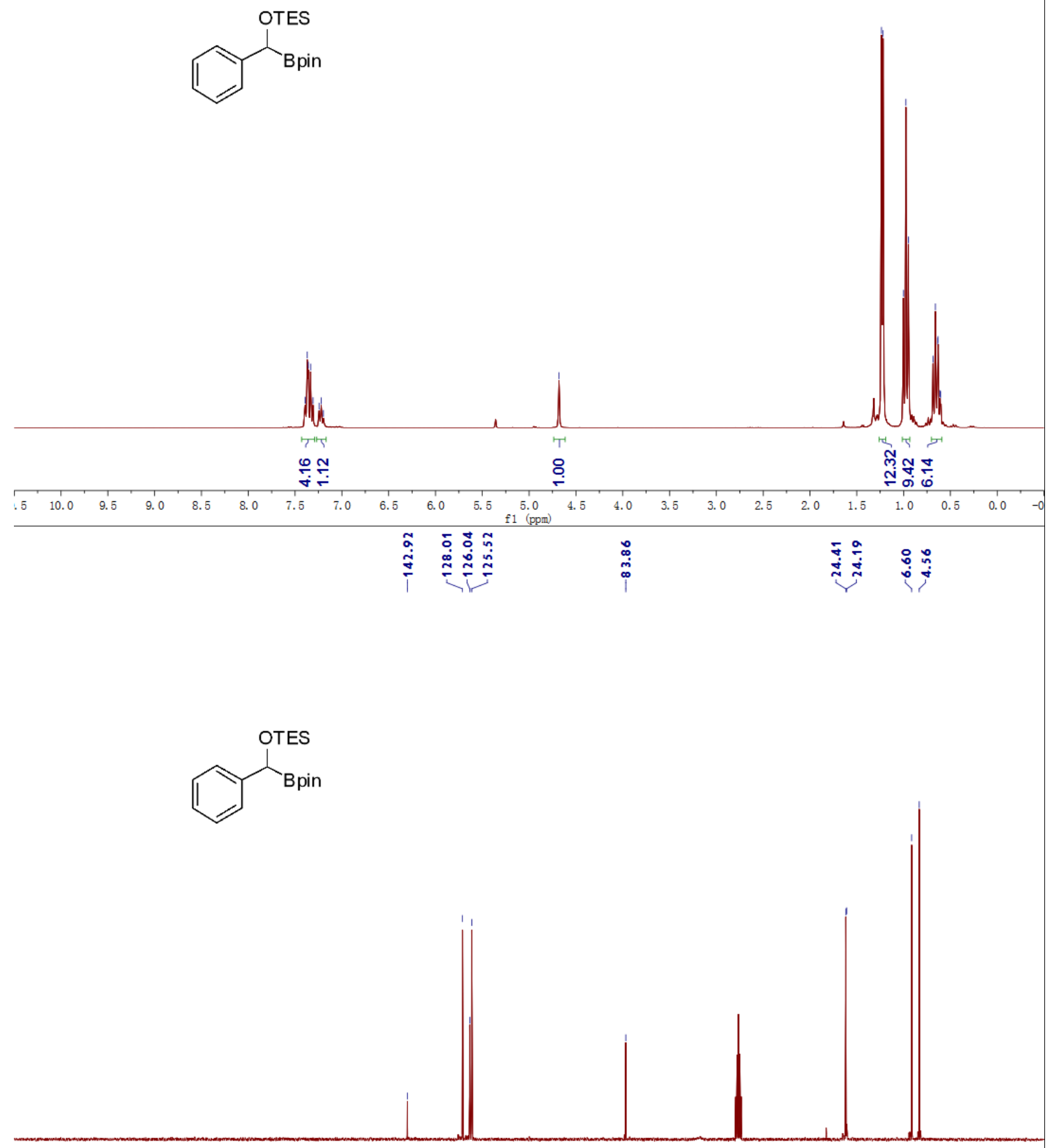

$\begin{array}{lllllllllllllllllllllllllllllllll}240 & 230 & 220 & 210 & 200 & 190 & 180 & 170 & 160 & 150 & 140 & 130 & 120 & 110 & 100 & 90 & 80 & 70 & 60 & 50 & 40 & 30 & 20 & 10 & 0 & -10 & -20\end{array}$ 


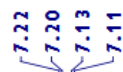

$\stackrel{i n}{i}$

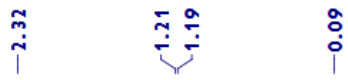
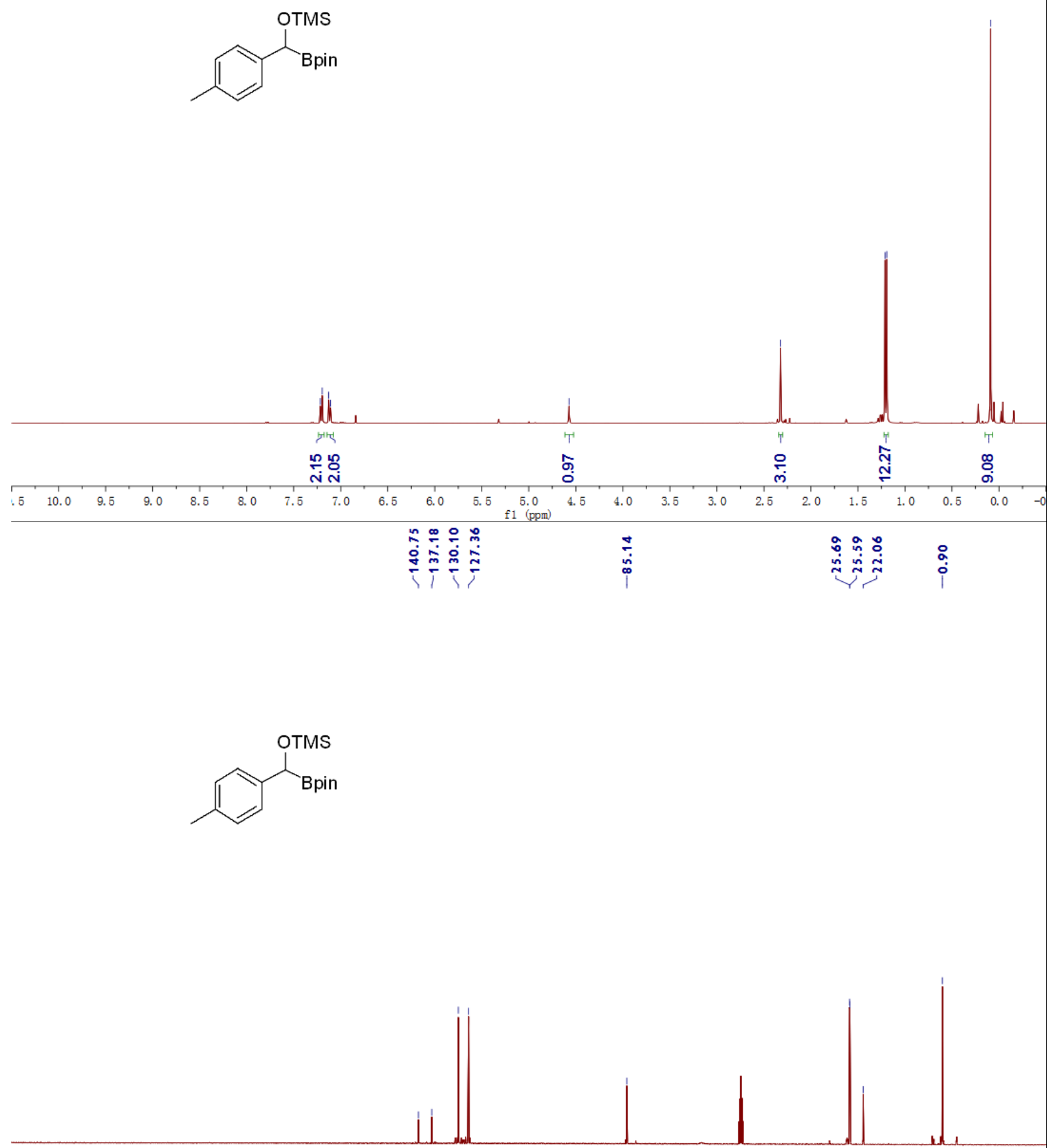

$\begin{array}{lllllllllllllllllllllllllllllll}240 & 230 & 220 & 210 & 200 & 190 & 180 & 170 & 160 & 150 & 140 & 130 & 120 & 110 & 100 & 90 & 80 & 70 & 60 & 50 & 40 & 30 & 20 & 10 & 0 & -10 & -20\end{array}$ 


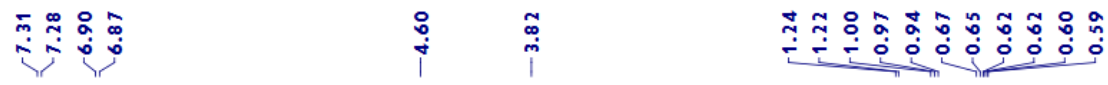

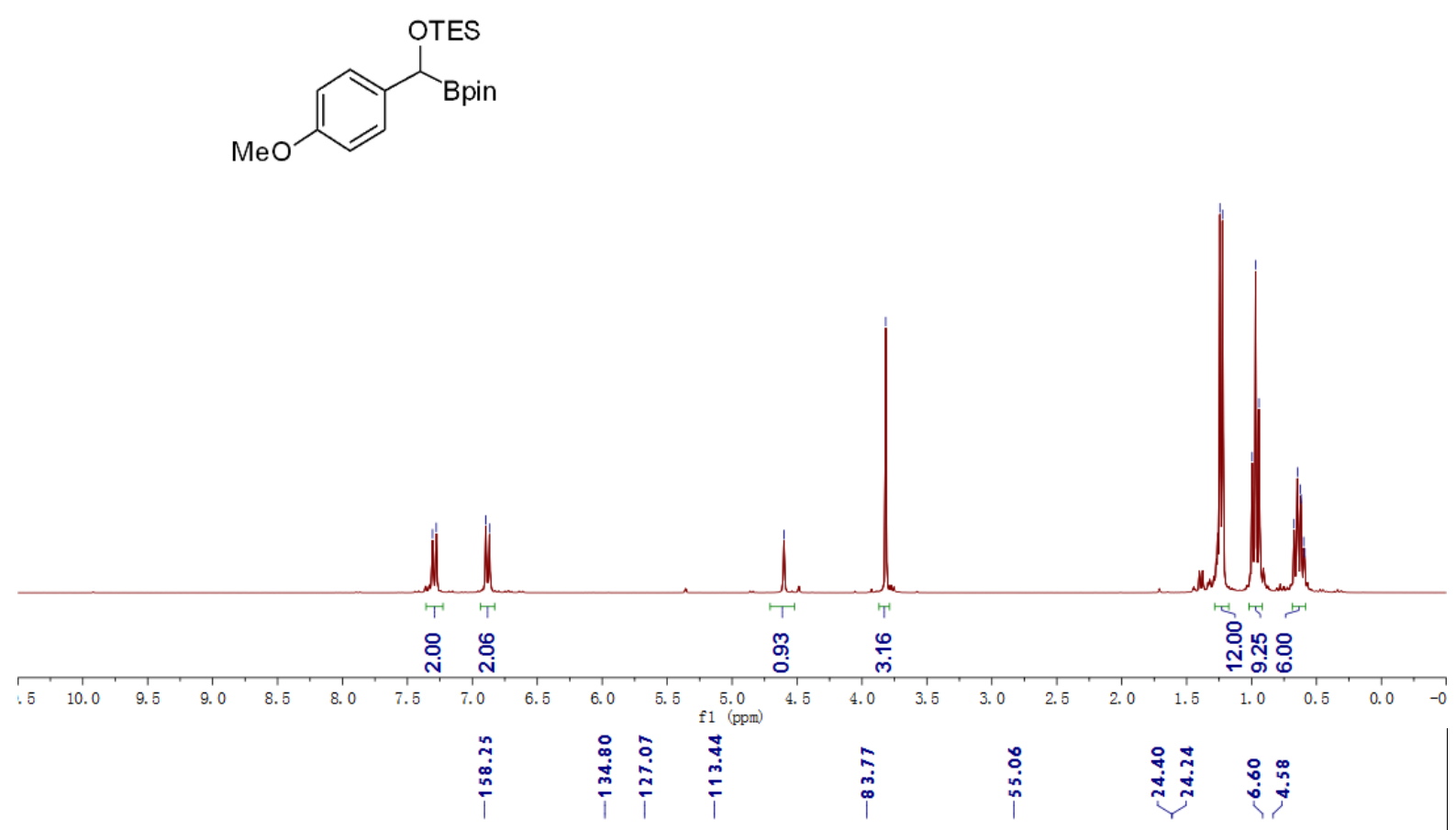<smiles>COc1ccc(C(Cc2ccccc2)OC)cc1</smiles>

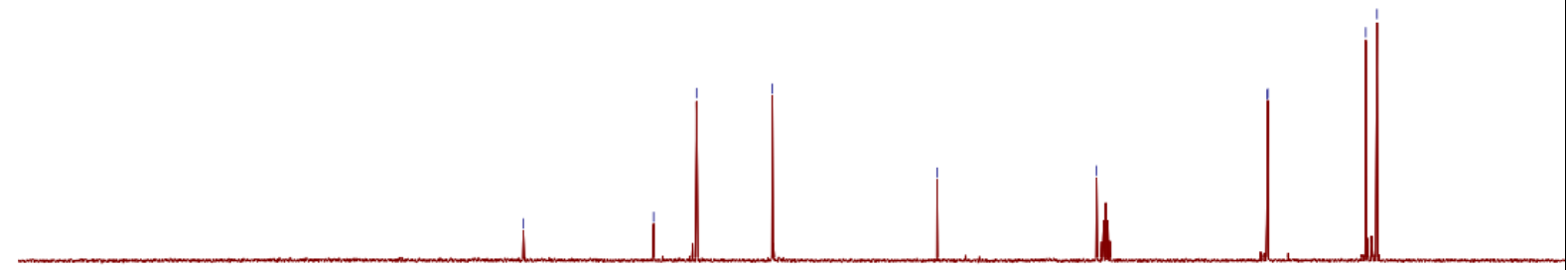


<smiles>COc1ccccc1C(OC)c1ccccc1</smiles>

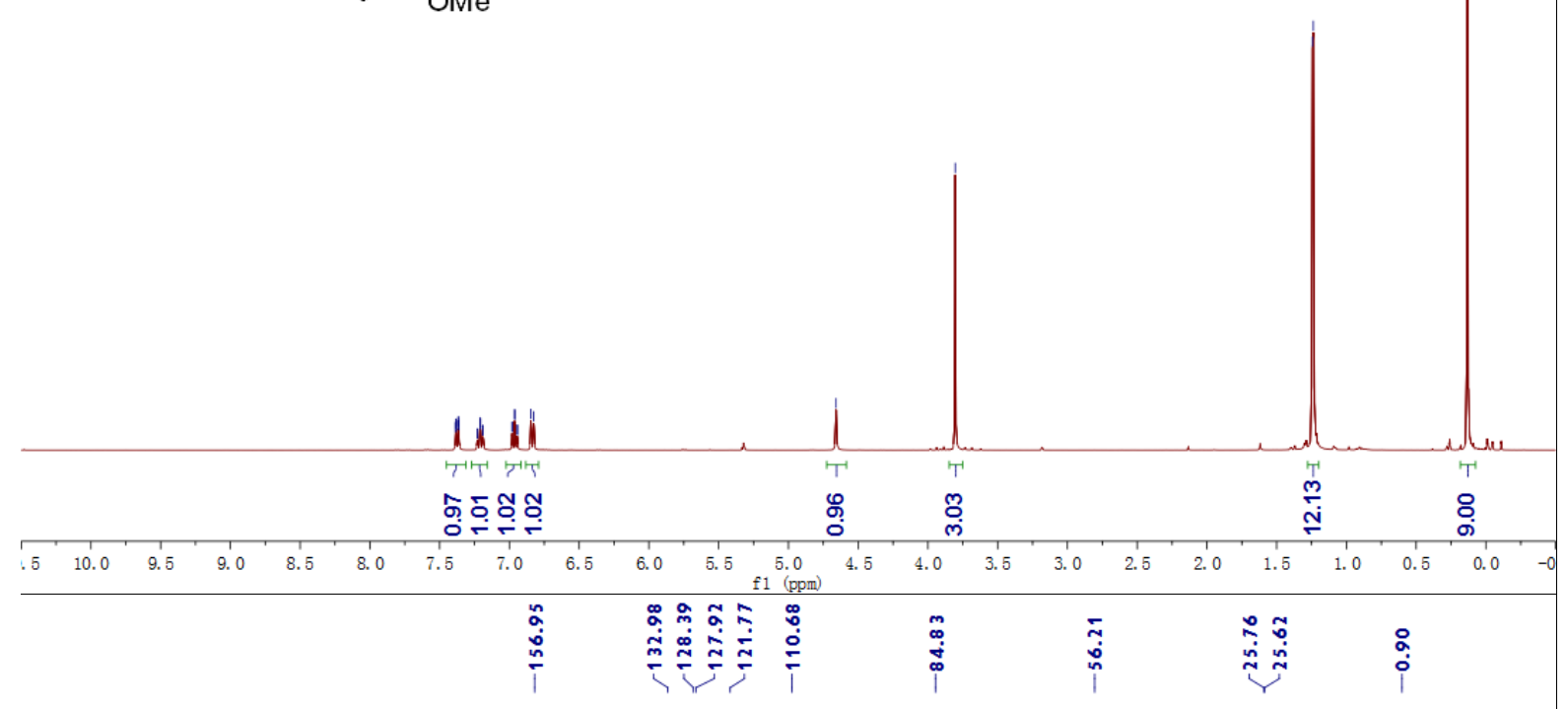<smiles>COc1ccccc1C(OC)c1ccccc1</smiles> 


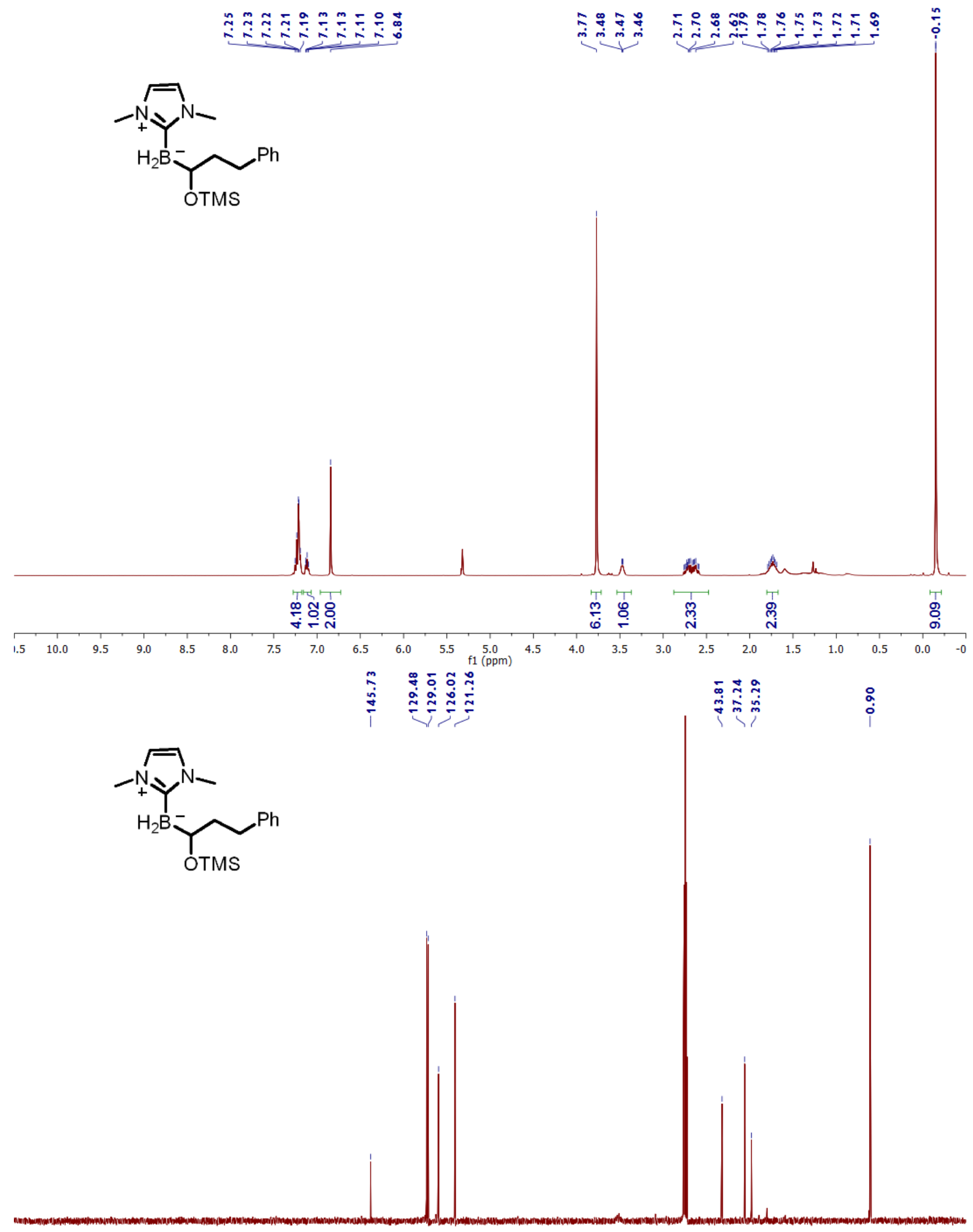

$\begin{array}{lllllllllllllllllllllllllllllll}240 & 230 & 220 & 210 & 200 & 190 & 180 & 170 & 160 & 150 & 140 & 130 & 120 & 110 & 100 & 90 & 80 & 70 & 60 & 50 & 40 & 30 & 20 & 10 & 0 & -10 & -20\end{array}$ 